\title{
NUMERICAL METHODS FOR CONSERVATION LAWS WITH ROUGH FLUX
}

\author{
H. HOEL, K. H. KARLSEN, N. H. RISEBRO, AND E. B. STORRØSTEN
}

ABSTRACT. Finite volume methods are proposed for computing approximate pathwise entropy/kinetic solutions to conservation laws with flux functions driven by low-regularity paths. For a convex flux, it is demonstrated that driving path oscillations may lead to "cancellations" in the solution. Making use of this property, we show that for $\alpha$-Hölder continuous paths the convergence rate of the numerical methods can improve from $\mathcal{O}\left(\mathrm{COST}^{-\gamma}\right)$, for some $\gamma \in[\alpha /(12-8 \alpha), \alpha /(10-6 \alpha)]$, with $\alpha \in(0,1)$, to $\mathcal{O}\left(\operatorname{COST}^{-\min (1 / 4, \alpha / 2)}\right)$. Numerical examples support the theoretical results.

\section{Contents}

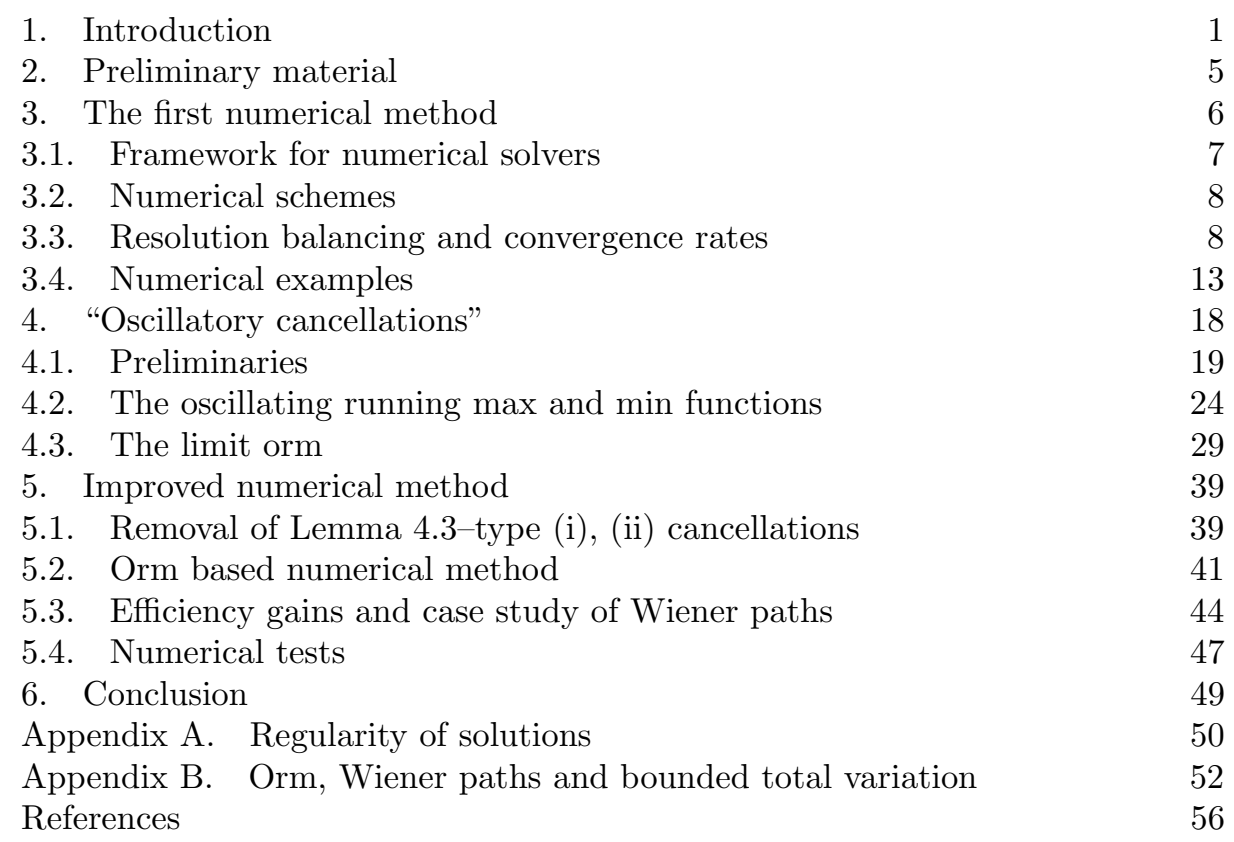

\section{INTRODUCTION}

The inclusion of random effects is important for the development of realistic models of physical phenomena. Frequently such models lead to nonlinear stochastic partial differential equations (SPDEs), whose solutions may possess singularities,

Date: December 2, 2018.

2010 Mathematics Subject Classification. Primary: 35L65, 65M06; Secondary: 60H15, 65C30.

Key words and phrases. Stochastic conservation law, rough time-dependent flux, pathwise entropy solution, finite difference method, convergence, stochastic numerics.

This work received supported by the Research Council of Norway through the project Stochastic Conservation Laws (250674/F20) and by the KAUST CRG4 Award Ref:2584. 
reflecting the appearance of shock waves, turbulence, or other physical features. Recently many researchers have targeted a wide range of questions relating to mathematical analysis and numerical methods for stochastic conservation laws and related SPDEs. Among the numerous questions addressed, we mention selection principles for singling out "correct" generalized solutions, theories of well-posedness (existence, uniqueness, stability of solutions), regularity and compactness properties (sometimes improved by the inclusion of noise), existence of invariant measures, and construction of convergent numerical methods.

Randomness can enter models in different ways, such as stochastic forcing or uncertain system parameters as well as random initial and boundary data. For example, a number of mathematical works [8, 11, 14, 19, 20, 21, 28, 48, 58, 155, 30, 77. 76] have studied the effect of Itô stochastic forcing on conservation laws,

$$
d u+\nabla \cdot f(u) \mathrm{d} t=\sigma(u) d W(t)
$$

where $f, \sigma$ are nonlinear functions and $W(t)$ is a (finite or infinite dimensional) Wiener process. Numerical methods, based on operator splitting [5, 50, 56] or finite volume discretizations [7, 6, 23, 24, 60, have been proposed and successfully analyzed for 1.1 and similar equations.

In another direction, several works [2, 31, 69, 71] have explored linear transport equations with low-regularity velocity coefficient $b(x)$ and "transportation noise",

$$
d u+b(x) \cdot \nabla u \mathrm{~d} t+\nabla u \circ d W(t)=0,
$$

where $\circ$ refers to the Stratonovich differential (integral).

In this work we are interested in constructing numerical methods for a nonlinear variant of 1.2 , namely

$$
d u+\nabla \cdot f(u) \circ d W(t)=0 .
$$

Nonlinear SPDEs like this were suggested and analyzed recently by Lions, Perthame, and Souganidis in a series of papers 62, 63, 64, where a pathwise well-posedness theory was developed based on entropy/kinetic solutions. Informally, their notion of solution is based on writing the kinetic formulation of $(1.3)$ :

$$
d \chi+f^{\prime}(\xi) \cdot \nabla \chi \circ d W(t)=\frac{\partial}{\partial \xi} m
$$

for a bounded measure $m(t, x, \xi) \geq 0$ and a function $u(t, x)$ (entropy solution) such that $\chi=\chi(t, x, \xi):=\chi(\xi, u(t, x))$, where

$$
\chi(\xi, u)= \begin{cases}+1 & \text { if } 0 \leq \xi \leq u \\ -1 & \text { if } u<\xi<0 \\ 0 & \text { otherwise. }\end{cases}
$$

The next step is to use a "transformation" to remove the noise term. This can be achieved by the "method of characteristics" since the previous equation is linear in $\chi$. The result is that the function

$$
v=v(t, x, \xi):=\chi\left(t, x+f^{\prime}(\xi) W(t), \xi\right)
$$

satisfies the following kinetic equation without a drift term:

$$
\begin{aligned}
\partial_{t} v= & \left(\frac{\partial}{\partial \xi} m\right)\left(t, x+f^{\prime}(\xi) W(t), \xi\right) \\
= & \frac{\partial}{\partial \xi}\left(m\left(t, x+f^{\prime}(\xi) W(t), \xi\right)\right) \\
& \quad-f^{\prime \prime}(\xi) W(t)\left(\frac{\partial}{\partial x} m\right)\left(t, x+f^{\prime}(\xi) W(t), \xi\right),
\end{aligned}
$$


where the right-hand side is "nonstandard". Informally, a weak solution to (1.4) is taken as the definition of a pathwise (entropy/kinetic) solution to (1.3), since (1.4) depends on the noise signal $W$ in a nice way ( $d W / d t$ is not entering the equation). Various results concerning existence, uniqueness, and "continuous dependence on the data" are found in the works [62, 64 by Lions, Perthame, and Souganidis (more on this below). The theory of pathwise solutions has been further developed by Gess and Souganidis in [36, 37, see also [49] and [4, 22] for a framework of intrinsic weak solutions of PDEs driven by rough signals, without relying on "transformation formulas" to remove the rough terms.

As alluded to above, we are interested in numerical solutions to conservation laws with rough time-dependent flux [1.3). To the best of our knowledge, 35] is the only work addressing numerical aspects of (1.3), but see Seeger's recent work 74] on monotone schemes for Hamilton-Jacobi equations. In the work [35], Gess, Perthame, and Souganidis prove convergence of a semi-discrete method based on Brenier's transport-collapse algorithm and path characteristics.

The primary goal of our work is to develop and analyze fully discrete and thus computable finite volume methods for solving the problem

$$
d u+\partial_{x} f(u) \circ d z=0 \quad \text { in }(0, T] \times \mathbb{R}, \quad u(0, \cdot)=u_{0},
$$

where $0<T<\infty$ is some fixed final time, $z:[0, T] \rightarrow \mathbb{R}$ is an $\alpha$-Hölder continuous driving path with $\alpha \in[0,1], f \in C^{2}(\mathbb{R})$, and $u_{0} \in\left(L^{1} \cap B V\right)(\mathbb{R})$. The basic numerical methods that we develop for 1.5 consist of the following two steps: (1) Approximate the driving path $z$ by a piecewise linear interpolant $z^{m}$ on a mesh over $[0, T]$ with $m-1$ degrees of freedom. (2) Solve (1.5) with driving signal $z^{m}$ using a traditional finite volume method for computing Kružkov entropy solutions, e.g., the Lax-Friedrichs, Godunov, or Engquist-Osher scheme 61]. The second step is justified by the observation that since $z$ is uniformly continuous, $z^{m}$ will be Lipschitz continuous for any fixed $m \in \mathbb{N}$, and for any Lipschitz path, classical and pathwise entropy solutions coincide, cf. Lemma 3.1 below. Several numerical examples are presented to illustrate the finite volume methods.

A continuous dependence estimate (cf. Theorem 2.1 below ) can be used to derive a convergence rate for the numerical methods. The result is a surprisingly slow rate of convergence: for any $\alpha \in(0,1]$ with $\left\|\dot{z}^{m}\right\|_{\infty}>\mathrm{cm}^{1-\alpha}$ for some $c>0$, the final time numerical error (measured in the $L^{1}$-norm) is bounded by

$$
\mathcal{O}\left(\operatorname{COST}^{-\gamma}\right), \quad \text { for some } \gamma \in\left[\frac{\alpha}{12-8 \alpha}, \frac{\alpha}{10-6 \alpha}\right] \text {. }
$$

Here COST denotes the computational cost of solving 1.5 with temporal and spatial resolution parameters $\Delta t$ and $\Delta x$ (that again are linked by the regularity of the driving path through the CFL condition); in other words, if the problem is solved numerically over the domain $[0, T] \times[a, b]$, then

$$
\operatorname{COST}(\Delta t, \Delta x)=\mathcal{O}\left(\frac{T}{\Delta t} \times \frac{b-a}{\Delta x}\right) .
$$

A conceptually helpful way of seeing why the convergence rate deteriorates so quickly as $\alpha$ decreases, justified by the CFL condition applied to the flux $\dot{z}^{m} f$, is to think of 1.5 as being integrated along the path $z^{m}$ rather than along time $t$. By that viewpoint the numerical error accumulates along the full path length of $z^{m}$ and leads to the replacement of the factor $T$ by $\left|z^{m}\right|_{B V([0, T])}$ in the standard error estimates for numerical methods for conservation laws [51, 61] (cf. also Section 2).

For strictly convex flux functions, the theory of generalized characteristics and Oleĭnik estimates can be used to derive a cancellation property due to path oscillations. We show that for any pathwise entropy solution $u(T)$ with piecewise linear 
path $z$, there exists a pathwise entropy solution $\tilde{u}(T)$ with a constructively defined "less oscillatory" path $\tilde{z}$ which is equivalent to $z$ in the sense that $u(T)=\tilde{u}(T)$, provided $u(0)=\tilde{u}(0)=u_{0}$.

The total variation of a driving path enters as a factor in the error estimate for the numerical methods (for details, see Section 2). In an effort to improve efficiency, we develop a variant of the numerical methods which solves 1.5 with $z$ replaced by the equivalent smoother path $\tilde{z}$. The theoretical efficiency gain by doing so can be significant. For instance, if the driving path is a realization of a standard Wiener process, then we show that the final time approximation error is bounded by

$$
\mathcal{O}\left(\left(\frac{\log (\mathrm{COST})}{\mathrm{COST}}\right)^{1 / 4}\right) \text {. }
$$

As sample paths of a standard Wiener process almost surely are $\alpha$-Hölder continuous for any $\alpha<1 / 2$, the improvement from (1.6) with $\alpha \approx 1 / 2$ to (1.7) is near-optimal in the sense that for conservation laws with $z(t)=t$, the optimal "cost versus accuracy" rate for finite volume methods is $-1 / 4$.

The cancellation property along with some of its theoretical consequences are further investigated in the companion work [46]. Although this article studies problem 1.5 from a numerical perspective and the companion work [46] is more focused on theoretical aspects, there are, in terms of results, some overlaps. Let us therefore point out a few characteristic features of the approach taken in the companion work [46] in relation to the one taken herein. Let $u$ be the pathwise entropy solution to 1.5 . The article 46 has the equivalence relation induced by the map $z \mapsto u(T)$ as its main object of study, and also as a fundamental tool. Proofs via the mentioned equivalence relation make continuous paths the natural objects of manipulation. In this work, a somewhat different approach is taken. In the case of a piecewise linear map, the solution map $u_{0} \mapsto u(T)$ is factorized as a product of solution operators, each associated to a straight line segment of the path, cf. (3.3) and (4.6). What amounts to manipulation of paths via equivalence relations in [46] is replaced by manipulations on the product of solution operators. The equivalent "less oscillatory" path $\tilde{z}$ is herein associated to an "irreducible factorization" of solution operators.

Although it is not a venue we will explore in this work, let us mention that the equation 1.5 may be extended to stochastic versions which are amenable to various forms of uncertainty quantification studies. To exemplify, let $\left(\Omega, \mathcal{F},(\mathcal{F})_{t \in[0, T]}, \mathbb{P}\right)$ denote a filtered probability space on which the standard Wiener process is defined, and consider 1.5 with the sampled driving path $z=W$. Then it follows from Theorem 2.1 that $u \in C\left([0, T] ; L^{1}(\mathbb{R})\right)$, almost surely. For a given functional $Q$ : $L^{1}(\mathbb{R}) \rightarrow \mathbb{R}$, one may for instance seek to approximate the quantity of interest

$$
\mathbb{E}[Q(u(T, \cdot))]=\int_{\Omega} Q(u(T, \cdot)) \mathbb{P}(d \omega) .
$$

The numerical methods developed in this paper are directly applicable to nonintrusive methods for approximating quantities of interest, e.g., Monte Carlo and Multilevel Monte Carlo methods. We refer to [38, 40, 1, 67, 68, 9, 73, 3, 78, 15] for recent developments on numerical methods for uncertainty quantification, and note that the contributions of this work share similarities with pathwise adaptive methods for conservation laws and stochastic differential equations, cf. [52, 44, 75, 42, 32, 47, 45, 39, 57, 79.

The remaining part of this paper is organized as follows: In Section 2, we collect some preliminary material, including a precise definition of pathwise solutions as well as relevant existence, uniqueness, and stability results. Section 3 presents finite volume methods for solving 1.5 with a general flux function $f$. Section 4 studies 
properties of oscillatory cancellations (for convex fluxes $f$ ) which we use to develop more efficient numerical methods in Section 5. Section 6 wraps the paper up with some concluding remarks.

\section{Preliminary material}

If we assume that the path $z$ is Lipschitz continuous $(\alpha=1)$, then $(1.5)$ reduces to a standard conservation law of the form

$$
\partial_{t} u+\partial_{x} f(u) \dot{z}=0 \quad \text { in }(0, T] \times \mathbb{R}, \quad u(0, \cdot)=u_{0},
$$

and, assuming for example that $u_{0} \in\left(L^{1} \cap B V\right)(\mathbb{R})$, well-posedness within the framework of Kružkov entropy solutions is a well-known result [18]. Furthermore, entropy solutions are equivalent to kinetic solutions 72 .

However, if $z$ is merely $\alpha$-Hölder continuous, for some $\alpha<1$, the well-posedness of entropy/kinetic solutions does not follow from standard arguments. This very fact motivates the following notion of solution [37, 62, 64, which can be viewed as a suitable weak formulation of 1.4 .

Definition 2.1. Assume $z \in C([0, T]), f \in C^{2}(\mathbb{R})$. Then $u \in\left(L^{1} \cap L^{\infty}\right)([0, T] \times \mathbb{R})$ is a pathwise entropy solution to equation (1.5) provided there exists a non-negative, bounded measure $m$ on $\mathbb{R} \times \mathbb{R} \times[0, T]$ such that for all $\rho_{0} \in C_{0}^{\infty}\left(\mathbb{R}^{2}\right)$ and $\rho$ given by

$$
\rho(x, y, \xi, \eta, t):=\rho_{0}\left(y-x+f^{\prime}(\xi) z(t), \xi-\eta\right),
$$

and all $\phi \in C_{0}^{\infty}([0, T])$,

$$
\begin{array}{r}
\int_{0}^{T} \partial_{t} \phi(r)(\rho * \chi)(y, \eta, r) d r+\phi(0)(\rho * \chi)(y, \eta, 0)-\phi(T)(\rho * \chi)(y, \eta, T) \\
=\int_{0}^{T} \phi(r) \partial_{\xi} \rho(x, y, \chi, \eta, r) m(x, \xi, r) d x d \xi d r
\end{array}
$$

where the "convolution along characteristics" term $\rho * \chi$ is defined by

$$
\rho * \chi(y, \eta, r):=\int \rho(x, y, \xi, \eta, r) \chi(x, \xi, r) d x d \xi .
$$

We note that for a continuous, piecewise Lipschitz path $z(t)$, the notions of entropy and pathwise entropy solutions coincide. We recall the following existence, uniqueness, and stability results for pathwise entropy solutions [62, Theorem 3.2].

Theorem 2.1. Let $u_{0} \in\left(L^{1} \cap L^{\infty}\right)(\mathbb{R})$ and assume $z \in C([0, T])$ and $f \in C^{2}(\mathbb{R})$. Then there exists a unique pathwise entropy solution $u \in C\left([0, T] ; L^{1}(\mathbb{R})\right)$ which satisfies the following inequality for all $p \in[1, \infty]$ :

$$
\sup _{t \in[0, T]}\|u(t)\|_{L^{p}(\mathbb{R})} \leq\left\|u_{0}\right\|_{L^{p}(\mathbb{R})} .
$$

Furthermore, if $u$ and $\bar{u}$ represent the pathwise entropy solution with respective paths $z$ and $\bar{z}$, then there exists a uniform constant $C>0$ such that for all $t \in[0, T]$,

$$
\begin{aligned}
\|u(t)-\bar{u}(t)\|_{1} & \leq\left\|u_{0}-\bar{u}_{0}\right\|_{1}+C\left[\left\|f^{\prime}\right\|\left(\left\|u_{0}\right\|_{B V}+\left\|\bar{u}_{0}\right\|_{B V}\right)|(z-\bar{z})(t)|\right. \\
& \left.+\sqrt{\sup _{s \in(0, t)}|(z-\bar{z})(s)|\left\|f^{\prime \prime}\right\|\left(\left\|u_{0}\right\|_{2}+\left\|\bar{u}_{0}\right\|_{2}\right)}\right] .
\end{aligned}
$$

Remark 2.1. Theorem 2.1 is proved in two steps. First, the result is verified for smooth paths $z \in C^{1}([0, T])$. Thereafter, the result is extended to $z \in C([0, T])$ by utilizing an approximation sequence $\left\{z^{m}\right\}_{m} \subset C^{1}([0, T])$ such that $z^{m} \rightarrow z$ in $C([0, T])$, using that solutions of 2.1 depend continuously on the driving path. 
Remark 2.2. According to Theorem 2.1, the pathwise entropy solution of 2.1 depends continuously on the rough path $z(t)$ in the supremum norm. It is also possible to prove a variant of 2.3 that includes continuous dependence with respect to the flux $f$. Such estimates are relevant for some numerical methods [51, 65]. Suppose $\bar{u}$ is the pathwise entropy solution of $(2.1)$ with the "data" $\left(u_{0}, z, f\right)$ replaced by $\left(\bar{u}_{0}, \bar{z}, \bar{f}\right)$. Then the "continuous dependence" estimate $(2.3)$ is replaced by

$$
\begin{aligned}
\|u(t, \cdot)-\bar{u}(t, \cdot)\|_{L^{1}(\mathbb{R})} \leq & \left\|u_{0}-\bar{u}_{0}\right\|_{L^{1}(\mathbb{R})} \\
& +C\left(t\left\|f^{\prime} z-\bar{f}^{\prime} \bar{z}\right\|_{L^{\infty}}+\sqrt{\left\|f^{\prime \prime} z-\bar{f}^{\prime \prime} \bar{z}\right\|_{L^{\infty}}}\right),
\end{aligned}
$$

for some constant $C$ depending on $\left\|\left(u_{0}, \bar{u}_{0}\right)\right\|_{L^{2} \cap B V}$. We omit the (lengthy) proof since the arguments are very similar to those found in [62. Earlier "deterministic" continuous dependence estimates can be found, e.g., in [13, 54, 65.

The numerical methods presented later are based on replacing the driving path $z(t)$ by a piecewise linear, Lipschitz continuous approximation $\bar{z}(t)$. Suppose for the moment that both paths $z(t), \bar{z}(t)$ are Lipschitz continuous. Then, adapting the arguments in [13, 54, 65, one can prove the following stability estimate:

$$
\begin{aligned}
\|u(t, \cdot)-\bar{u}(t, \cdot)\|_{L^{1}(\mathbb{R})} \leq \| & u_{0}-\bar{u}_{0} \|_{L^{1}(\mathbb{R})} \\
& +C\left(\left\|f^{\prime}-\bar{f}^{\prime}\right\|_{L^{\infty}}+\|\dot{z}-\dot{\bar{z}}\|_{L^{1}((0, t))}\right),
\end{aligned}
$$

where the constant $C$ depends on the data as follows:

$$
C=C\left(\left\|\left(u_{0}, \bar{u}_{0}\right)\right\|_{B V},\left\|\left(f^{\prime}, \bar{f}^{\prime}\right)\right\|_{L^{\infty}},\|(\dot{z}, \dot{\bar{z}})\|_{L^{1}((0, t))}\right) .
$$

At variance with 2.4, note that the estimate 2.5 does not depend on the second derivative of the flux, but it does depend on the derivative of the path (actually the total variation of the path). Consequently, there is a trade-off between the regularity of the nonlinear flux function and the regularity of the path.

\section{THE FIRST NUMERICAL METHOD}

In this section we describe numerical methods for 1.5 . Convergence rates are derived and a few numerical examples are presented to illustrate the qualitative behavior of solutions.

Since solutions to 1.5 depend on the differential of the driving path $z$, but not on its initial value $z(0)$, we may without loss of generality restrict ourselves to driving paths in the function space

$$
C_{0}([0, T]):=\{g \in C([0, T]) \mid g(0)=0\} .
$$

Denote the set of $\alpha$-Hölder continuous functions on $[0, T]$ that are zero-valued at $t=0$ by

$$
C_{0}^{0, \alpha}([0, T]):=\left\{z \in C_{0}([0, T]) \mid \sup _{s \neq t \in[0, T]} \frac{|z(s)-z(t)|}{|t-s|^{\alpha}}<\infty\right\}, \quad \alpha \in(0,1] .
$$

The set of Lipschitz continuous functions on $[0, T]$ that are zero-valued at $t=0$ are denoted by

$$
\operatorname{Lip}_{0}([0, T]):=C_{0}^{0,1}([0, T]) .
$$

Given a mesh

$$
0=\tau_{0}<\tau_{1}<\cdots<\tau_{m}=T, \quad m \geq 2,
$$


we introduce the set of functions which are Lipschitz continuous over $[0, T]$ and linear over each interval $\left[\tau_{k}, \tau_{k+1}\right]$, i.e.,

$$
\begin{array}{r}
I_{0}\left([0, T] ;\left\{\tau_{j}\right\}_{j=0}^{m}\right):=\left\{g \in \operatorname{Lip}_{0}([0, T])|g|_{\left[\tau_{k}, \tau_{k+1}\right]}(t)=g\left(\tau_{k}\right)+\frac{t-\tau_{k}}{\Delta \tau}\left(g\left(\tau_{k+1}\right)-g\left(\tau_{k}\right)\right)\right. \\
\quad \text { for all } k=0,1, \ldots, m-1\} .
\end{array}
$$

We also introduce the operator $\mathcal{I}[\cdot]\left(\cdot ;\left\{\tau_{j}\right\}_{j=0}^{m}\right): C_{0}([0, T]) \rightarrow I_{0}\left([0, T] ;\left\{\tau_{j}\right\}_{j=0}^{m}\right)$ defined by

$$
\begin{aligned}
\mathcal{I}[g]\left(t ;\left\{\tau_{j}\right\}_{j=0}^{m}\right):= & \mathbf{1}_{\left[\tau_{0}, \tau_{1}\right]}(t)\left(g\left(\tau_{0}\right)+\frac{t-\tau_{0}}{\Delta \tau}\left(g\left(\tau_{1}\right)-g\left(\tau_{0}\right)\right)\right) \\
& +\sum_{k=1}^{m-1} \mathbf{1}_{\left(\tau_{k}, \tau_{k+1}\right]}(t)\left(g\left(\tau_{k}\right)+\frac{t-\tau_{k}}{\Delta \tau}\left(g\left(\tau_{k+1}\right)-g\left(\tau_{k}\right)\right)\right),
\end{aligned}
$$

for $g \in C_{0}([0, T])$. On some occasions we use the shorthand notations $I_{0}^{m}([0, T])=$ $I_{0}\left([0, T] ;\left\{\tau_{j}\right\}_{j=0}^{m}\right)$ and $\mathcal{I}^{m}[\cdot]=\mathcal{I}[\cdot]\left(\cdot ;\left\{\tau_{j}\right\}_{j=0}^{m}\right)$.

3.1. Framework for numerical solvers. We propose the following numerical method for solving (1.5):

(i) For an appropriately chosen mesh $\left\{\tau_{j}\right\}_{j=0}^{m}$, approximate the driving path $z \in C_{0}([0, T])$ by the piecewise linear interpolant $z^{m}:=\mathcal{I}^{m}[z]$.

(ii) Solve (2.1) with the driving path $z$ replaced by the Lipschitz path $z^{m}$, using a consistent, conservative and monotone finite volume method (for entropy solutions).

With the purpose of studying properties of the entropy solution of 1.5 with path $z^{m}$, we introduce the solution operator $\mathcal{S} \cdot(\cdot)$. mapping $\mathbb{R} \times[0, \infty) \times\left(L^{1} \cap B V\right)(\mathbb{R})$ into $\left(L^{1} \cap B V\right)(\mathbb{R})$. For $\kappa \in \mathbb{R}, s \geq 0$ and $v \in\left(L^{1} \cap B V\right)(\mathbb{R}), \mathcal{S}^{\kappa}(s) v$ denotes the solution at time $t=s$ of

$$
\partial_{t} u+\kappa \partial_{x} f(u)=0 \quad \text { in }(0, \infty) \times \mathbb{R}, \quad u(0, \cdot)=v .
$$

Using the convention that for any $k=0,1, \ldots, m-1, \dot{z}_{k}^{m}:=\lim _{t \downarrow} \tau_{k} \dot{z}^{m}(t)$ and denoting $\Delta \tau_{k}=\tau_{k+1}-\tau_{k}$, we define

$$
u^{m}(t, \cdot)= \begin{cases}u_{0} & \text { if } t=0, \\ \mathcal{S}^{\dot{z}_{0}^{m}}(t) u_{0} & \text { if } t \in\left(\tau_{0}, \tau_{1}\right], \\ \mathcal{S}^{\dot{z}_{1}^{m}}\left(t-\tau_{1}\right) \mathcal{S}^{\dot{z}_{0}^{m}}\left(\Delta \tau_{0}\right) u_{0} & \text { if } t \in\left(\tau_{1}, \tau_{2}\right], \\ \vdots & \\ \mathcal{S}^{\dot{z}_{m-1}^{m}}\left(t-\tau_{m-1}\right) \mathcal{S}^{\dot{z}_{m-2}^{m}}\left(\Delta \tau_{m-2}\right) \ldots \mathcal{S}^{\dot{z}_{0}^{m}}\left(\Delta \tau_{0}\right) u_{0} & \text { if } t \in\left(\tau_{m-1}, T\right] .\end{cases}
$$

To justify step (ii) of the above algorithm, let us verify that $u^{m}$ is a Kružkov entropy solution as well as a pathwise entropy solution.

Lemma 3.1. Assume that $u_{0} \in\left(L^{1} \cap B V\right)(\mathbb{R}), f \in C^{2}(\mathbb{R}), z \in C_{0}([0, T])$ and $z^{m} \in \mathcal{I}^{m}[z]$. Then the function $u^{m}$ defined by equation (3.3) is a Kružkov entropy solution of 2.1) with driving path $z^{m}$, such that

$$
u^{m} \in C\left([0, T] ; L^{1}(\mathbb{R})\right) \text { and }\left|u^{m}(t)\right|_{\mathrm{BV}(\mathbb{R})} \leq\left|u_{0}\right|_{\mathrm{BV}(\mathbb{R})} \forall t \in[0, T] .
$$

Moreover, $u^{m}$ is also a pathwise entropy solution of 1.5 with driving path $z^{m}$.

Proof. It is enough to remark that $u^{m}$ is a Kružkov entropy solution of 2.1p. By 62 it is then also a pathwise entropy solution of $[1.5]$, as the total variation of $z^{m}$ 
is finite. Indeed, by construction, on each time interval $\left[\tau_{k}, \tau_{k+1}\right], u^{m}$ satisfies the following local Kružkov entropy condition:

$$
\begin{aligned}
& \int_{\tau_{k}}^{\tau_{k+1}} \int_{\mathbb{R}}\left|u^{m}-c\right| \phi_{t}+\operatorname{sign}(u-c) \dot{z}^{m}\left(f\left(u^{m}\right)-f(c)\right) \phi_{x} \mathrm{~d} x \mathrm{~d} t \\
& +\int_{\mathbb{R}}\left|u^{m}\left(\tau_{k}+, x\right)-c\right| \phi\left(\tau_{k}, x\right)-\left|u^{m}\left(\tau_{k+1}-, x\right)-c\right| \phi\left(\tau_{k+1}, x\right) \mathrm{d} x \geq 0,
\end{aligned}
$$

for all $c \in \mathbb{R}$ and test functions $\phi \geq 0$. Since $u^{m} \in C_{0}\left([0, T] ; L^{1}(\mathbb{R})\right)$, we have that $u\left(\tau_{k}+\right)=u\left(\tau_{k}-\right)$ in $L^{1}(\mathbb{R})$-sense, and summing over $k=0, \ldots, m-1$ gives that $u^{m}$ is a Kružkov entropy solution on $[0, T]$. See [51] for verification of 3.4 .

3.2. Numerical schemes. Let $U$ denote a finite volume method approximation of $u^{m}$ with uniform spatial and temporal mesh parameters $\Delta x$ and $\Delta t$ such that

$$
U_{j}^{n} \approx \frac{1}{\Delta x} \int_{x_{j}-\Delta x / 2}^{x_{j}+\Delta x / 2} u^{m}\left(t_{n}, y\right) \mathrm{d} y, \quad t_{n}=n \Delta t, x_{j}=j \Delta x .
$$

Although theoretical results will be stated in more generality, we have in the numerical implementations restricted ourselves to two numerical methods: the LaxFriedrichs scheme

$$
U_{j}^{n+1}=\frac{U_{j+1}^{n}+U_{j-1}^{n}}{2}-\frac{\dot{z}^{m}\left(t_{n}\right) \Delta t}{2 \Delta x}\left(f\left(U_{j+1}^{n}\right)-f\left(U_{j-1}^{n}\right)\right),
$$

and the Engquist-Osher scheme

$$
\begin{aligned}
U_{j}^{n+1}=U_{j}^{n}-\frac{\dot{z}^{m}\left(t_{n}\right) \Delta t}{2 \Delta x}( & f\left(U_{j+1}^{n}\right)-f\left(U_{j-1}^{n}\right) \\
& \left.\quad-\operatorname{sign}\left(\dot{z}^{m}\left(t_{n}\right)\right)\left(\int_{U_{j}^{n}}^{U_{j+1}^{n}}\left|f^{\prime}(s)\right| d s-\int_{U_{j-1}^{n}}^{U_{j}^{n}}\left|f^{\prime}(s)\right| d s\right)\right) .
\end{aligned}
$$

If volume averages of $u_{0}$ are computable, both schemes are initialized by setting

$$
U_{j}^{0}:=\frac{1}{\Delta x} \int_{x_{j}-\Delta x / 2}^{x_{j}+\Delta x / 2} u_{0}(y) d y,
$$

otherwise each volume average of $u_{0}$ is approximated using a finite number of quadrature points evaluating the càdlàg modification of $u_{0}$ over each volume.

For a consistent treatment of $\dot{z}^{m}$ at (possible) discontinuity points $\left\{\tau_{j}\right\}$, we will always assume that $\left\{\tau_{j}\right\} \subset\left\{t_{n}\right\}$, i.e., the interpolation points of $z^{m}$ constitute a subset of the temporal mesh points used in the finite volume scheme.

We refer to [51, 61] for background material on numerical methods for conservation laws.

3.3. Resolution balancing and convergence rates. Assuming that the mesh $\left\{\tau_{j}\right\} \subset[0, T]$ consists of uniformly spaced points, the numerical solution $U_{j}^{n}$ defined above has three "resolution parameters": the path interpolation step size $\Delta \tau$, and the temporal/spatial mesh sizes $\Delta t$ and $\Delta x$ of the finite volume method. To construct an efficient and stable (convergent) numerical method, these parameters must be appropriately balanced. In this section, we derive a convergence rate expressed in terms of the resolution parameters, and determine the optimal balance for minimizing the error in terms of computational cost.

The next lemma contains our first convergence rate result.

Lemma 3.2. Let $u \in C\left([0, T] ; L^{1}(\mathbb{R})\right)$ denote the unique pathwise entropy solution of (1.5) for given $u_{0} \in\left(L^{1} \cap B V\right)(\mathbb{R}), f \in C^{2}(\mathbb{R})$, and $z \in C_{0}^{0, \alpha}([0, T])$ with $\alpha \in(0,1]$. Assume that $\left\{\tau_{j}\right\}_{j=0}^{m} \subset[0, T]$ are uniformly spaced points with step 
size $\Delta \tau=T / m$, and that for the numerical solution $U$ defined in Section 3.1 the following global CFL condition is fulfilled:

$$
\frac{\Delta t\left\|\dot{z}^{m}\right\|_{\infty}\left\|f^{\prime}\right\|_{\infty}}{\Delta x} \leq C_{\mathrm{CFL}}
$$

where the constant $C_{\mathrm{CFL}}>0$ depends on the scheme used. Then

$$
\|u(T)-U(T)\|_{1} \leq\left\|u_{0}-U(0)\right\|_{1}+C\left(\Delta \tau^{\alpha / 2}+\Delta \tau^{\alpha-1}(\sqrt{\Delta t}+\sqrt{\Delta x})\right),
$$

where $C>0$ is independent of the resolution parameters.

Proof. Recall that for the given initial data $u_{0}$ and flux $f, u^{m}$ denotes the pathwise entropy solution with driving signal $z^{m}$, and $U$ denotes the corresponding numerical solution with path $z^{m}$. By the triangle inequality and (2.3),

$$
\begin{aligned}
\|u(T)-U(T)\|_{1} & \leq\left\|u(T)-u^{m}(T)\right\|_{1}+\left\|u^{m}(T)-U(T)\right\|_{1} \\
& \leq C \sqrt{\left\|z-z^{m}\right\|_{\infty}}+\left\|u^{m}(T)-U(T)\right\|_{1} .
\end{aligned}
$$

The error can thus be bounded by the sum of the path approximation error and the finite volume discretization error. Since $z \in C_{0}^{0, \alpha}([0, T])$ and $z^{m}$ uses $m+1$ uniformly spaced interpolation points with step size $\Delta \tau$,

$$
\left\|z-z^{m}\right\|_{\infty}=\mathcal{O}\left(\Delta \tau^{\alpha}\right) \quad \text { and } \quad\left\|\dot{z}^{m}\right\|_{\infty}=\mathcal{O}\left(\Delta \tau^{\alpha-1}\right) .
$$

To bound the second term, we repeat the proof of Kuznetsov's lemma (see e.g. [51, with $f$ replaced by $\dot{z}^{m} f$ ) to derive that for some constant $C$, depending on $\left\|f^{\prime}\right\|_{\infty}$ and $\left|u_{0}\right|_{B V(\mathbb{R})}$, the following error estimate holds for any consistent, conservative and monotone finite volume approximation:

$$
\begin{aligned}
\left\|u^{m}(T)-U(T)\right\|_{1} & \leq\left\|u_{0}^{m}-U(0)\right\|_{1}+C\left\|\dot{z}^{m}\right\|_{\infty}(\sqrt{\Delta t}+\sqrt{\Delta x}) \\
& \leq\left\|u_{0}^{m}-U(0)\right\|_{1}+C \Delta \tau^{\alpha-1}(\sqrt{\Delta t}+\sqrt{\Delta x}) .
\end{aligned}
$$

Having obtained a convergence rate expressed in terms of the resolution parameters, we next seek to optimally balance these parameters for the purpose of minimizing computational cost versus accuracy. Let us first discuss briefly how the spatial support of the numerical solutions grows in time.

For any $y \in \mathbb{R}$, let $\lceil y\rceil$ denote the smallest $n \in \mathbb{Z}$ such that $n \geq y$. For two functions $g_{1}$ and $g_{2}$ we use the notation $g_{1}(m)=\Theta\left(g_{2}(m)\right)$ to signify that there exists two positive constants $C_{1}$ and $C_{2}$ such that $C_{1} g_{1}(m) \leq g_{2}(m) \leq C_{2} g_{1}(m)$ for all $m$, in particular $g_{1}(m)=\Theta\left(g_{2}(m)\right)$ implies that $g_{1}(m)=\mathcal{O}\left(g_{2}(m)\right)$ and $g_{2}(m)=\mathcal{O}\left(g_{1}(m)\right)$. Let $N=\lceil T / \Delta t\rceil$ denote the number of timesteps used in the finite volume method (expressing that number by $N$ rather than $\lceil T / \Delta t\rceil$ simplifies the transition to non-uniform timesteps $\Delta t_{n}$ later on).

Suppose that at some time $t_{n} \in[0, T-\Delta t]$, we have

$$
-\infty<a=\inf \left\{x \in \mathbb{R} \mid U\left(t_{n}, x\right) \neq 0\right\} \quad \text { and } \quad \sup \left\{x \in \mathbb{R} \mid U\left(t_{n}, x\right) \neq 0\right\}=b<\infty .
$$

Computing $U\left(t_{n+1}\right)$ from $U\left(t_{n}\right)$ by a $k$-stencil numerical scheme yields

$$
-\infty<a-k \Delta x \leq \inf \left\{x \in \mathbb{R} \mid U\left(t_{n+1}, x\right) \neq 0\right\}
$$

and

$$
\sup \left\{x \in \mathbb{R} \mid U\left(t_{n+1}, x\right) \neq 0\right\} \leq b+k \Delta x<\infty .
$$

Let $\operatorname{Leb}(\cdot)$ denote the Lebesgue measure on $(\mathbb{R}, \mathcal{B})$ and for any $g \in L^{1}(\mathbb{R})$ let $\operatorname{supp}(g)$ denote the essential support of $g$. Based on the above observations, we 
will in the sequel assume that for any $u_{0} \in\left(L^{1} \cap B V\right)(\mathbb{R})$ with Leb $\left(\operatorname{supp}\left(u_{0}\right)\right)>0$, $z^{m}=\mathcal{I}^{m}[z]$ and $f \in C^{2}(\mathbb{R})$, there exists constants $c_{1}, c_{2}>0$ such that

$$
c_{1} \leq \operatorname{Leb}\left(\bigcup_{k \in\{0,1, \ldots, N\}} \operatorname{supp}\left(U\left(t_{k}\right)\right)\right) \leq c_{2}(1+N \Delta x)
$$

Note that in the classical setting $\alpha \geq 1$, the CFL condition (3.6) allows for $\Delta t=$ $\Theta(\Delta x)$. This yields $N=\Theta\left(\Delta x^{-1}\right)$, and assumption 3.10 becomes

$$
\operatorname{Leb}\left(\bigcup_{k \in\{0,1, \ldots, N\}} \operatorname{supp}\left(U\left(t_{k}\right)\right)\right)=\Theta(1),
$$

indicating finite speed of propagation. If $\alpha<1$, however, then the CFL condition imposes the constraint $N \Delta x \geq C\left\|\dot{z}^{m}\right\|_{\infty}$. So whenever $\lim _{m \rightarrow \infty}\left\|\dot{z}^{m}\right\|_{\infty}=\infty$, a numerical solution generated by a scheme with artificial diffusion may attain infinite speed of propagation in the limit as $m \rightarrow \infty$ (although this is not an issue with the numerical examples presented later).

Theorem 3.3. Let $u \in C\left([0, T] ; L^{1}(\mathbb{R})\right)$ be the unique pathwise entropy solution of 1.5$)$, for given $u_{0} \in\left(L^{1} \cap B V\right)(\mathbb{R})$ with Leb $\left(\operatorname{supp}\left(u_{0}\right)\right)<\infty, f \in C^{2}(\mathbb{R})$ with $\left\|f^{\prime}\right\|_{\infty}>0$, and $z \in C_{0}^{0, \alpha}([0, T])$ with $\alpha \in(0,1]$. For any $m \geq 2$, let $\left\{\tau_{j}\right\}_{j=0}^{m} \subset[0, T]$ denote the uniform mesh with step size $\Delta \tau=T / m$ and assume the computational cost of generating the interpolant $z^{m}=\mathcal{I}^{m}[z]$ is $\Theta\left(m^{\beta}\right)$ for some $\beta \geq 1$, and that there exists an $\check{m} \geq 2$ such that

$$
\left\|\dot{z}^{m}\right\|_{\infty}>m^{(1-\alpha) / 3} \quad \forall m \geq \check{m} .
$$

Let $U$ denote a numerical solution linked to the two-step algorithm in Section 3.1. satisfying the CFL condition

$$
\Delta t=\frac{\Delta \tau}{\max \left(\left\lceil\frac{\max \left(\left\{\left|\Delta z_{k}^{m}\right|\right\}_{k=0}^{m-1}\right)\left\|f^{\prime}\right\|_{\infty}}{C_{\mathrm{CFL}} \Delta x}\right\rceil, 1\right)} .
$$

Assume that the spatial support of $U([0, T])$ is covered by an interval $\left[a_{m}, b_{m}\right] \subset \mathbb{R}$ that satisfies

$$
c_{1} \leq b_{m}-a_{m} \leq c_{2}(1+N \Delta x),
$$

for some $c_{1}, c_{2}>0$, cf. 3.10 .

Then the optimal balance of resolution parameters for minimizing computational cost versus accuracy is

$$
\Delta x=\Theta\left(\frac{\Delta \tau^{\alpha}}{\left\|\dot{z}^{m}\right\|_{\infty}^{2}}\right) \quad \text { and } \quad \Delta t=\Theta\left(\frac{\Delta \tau^{\alpha}}{\left\|\dot{z}^{m}\right\|_{\infty}^{3}}\right),
$$

and

$$
\|u(T)-U(T)\|_{1}=\mathcal{O}\left(m^{-\alpha / 2}\right)
$$

is achieved at the computational cost

$$
\hat{c}_{1}\left(\left\|\dot{z}^{m}\right\|_{\infty}^{5} m^{2 \alpha}+m^{\beta}\right) \leq \operatorname{Cost}(U) \leq \hat{c}_{2}\left(\left\|\dot{z}^{m}\right\|_{\infty}^{6} m^{2 \alpha}+m^{\beta}\right),
$$

for some $\hat{c}_{1}, \hat{c}_{2}>0$.

Proof. Assume that $m \geq \check{m}$. Then the CFL condition (3.11) imposes the following constraint on the timestep:

$$
\Delta t=\Theta\left(\frac{\Delta x}{\left\|\dot{z}^{m}\right\|_{\infty}}\right)
$$


Since $u^{m}(0)=u_{0} \in\left(L^{1} \cap B V\right)(\mathbb{R})$, the approximation of the initial data (3.5) yields that

and by 3.8 ,

$$
\left\|u_{0}^{m}-U(0)\right\|_{1}=\mathcal{O}(\Delta x)
$$

$$
\|u(T)-U(T)\|_{1}=\mathcal{O}\left(\Delta \tau^{\alpha / 2}+\left\|\dot{z}^{m}\right\|_{\infty} \sqrt{\Delta x}\right)
$$

The optimal balance of resolution parameters for minimizing the computational cost versus accuracy is achieved through equilibration of error contributions:

$$
\Delta x=\Theta\left(\frac{\Delta \tau^{\alpha}}{\left\|\dot{z}^{m}\right\|_{\infty}^{2}}\right) .
$$

Since $m \geq \check{m}$,

and thus

$$
\frac{\Delta x}{\left\|\dot{z}^{m}\right\|_{\infty}}=\Theta\left(\frac{\Delta \tau^{\alpha}}{\left\|\dot{z}^{m}\right\|_{\infty}^{3}}\right)=\mathcal{O}(\Delta \tau)
$$

$$
\Delta t=\Theta\left(\min \left(\frac{\Delta x}{\left\|\dot{z}^{m}\right\|_{\infty}}, \Delta \tau\right)\right)=\Theta\left(\frac{\Delta \tau^{\alpha}}{\left\|\dot{z}^{m}\right\|_{\infty}^{3}}\right)
$$

The computational cost of the numerical solution $U(T)$ is the sum of $\Theta\left(m^{\beta}\right)$ for generating the piecewise linear interpolant $z^{m}$, and

$$
\Theta\left(\frac{T}{\Delta t} \times \frac{b_{m}-a_{m}}{\Delta x}\right)
$$

for solving $U$ over $[0, T] \times\left[a_{m}, b_{m}\right]$.

Remark 3.1. Theorem 3.3 provides a surprisingly slow convergence rate. For instance, if $z \in C_{0}^{0,1 / 2}([0, T])$ and $\left\|\dot{z}^{m}\right\|_{\infty}=\Theta\left(m^{1 / 2}\right)$, then 3.12 implies that in order to achieve the accuracy $\|u(T)-U(T)\|_{L^{1}}=\mathcal{O}(\epsilon)$, one needs $m \geq c \epsilon^{-4}$ for some $c>0$. By (3.13), this results in the astounding $\Theta\left(\epsilon^{-14}+\epsilon^{-4 \beta}\right)$ lower bound on the computational cost. In some numerical experiments, however, we observe a better convergence rate than predicted by 3.12 , see Example 3.2 in Section 3.4 .

Remark 3.2. In Theorem 3.3, we assume the computational cost of generating/sampling the piecewise linear interpolant $z^{m}$ is $\Theta\left(m^{\beta}\right)$ for some $\beta \geq 1$. If $z$ is a realization of a Wiener process, for instance, then $\beta=1$, but to cover the more general Hölder continuous stochastic processes, we allow for $\beta \geq 1$.

We next consider the use of an adaptive mesh $\left\{t_{n}\right\}_{n=0}^{N} \supset\left\{\tau_{k}\right\}_{k=0}^{m}$ that have uniform timesteps over each interpolation interval $\left[\tau_{k}, \tau_{k+1}\right]$ for $k=0,1, \ldots, m-1$. That is, $t_{0}=0$ and given $t_{n} \in\left[\tau_{k}, \tau_{k+1}\right)$, the next mesh point is set to

$$
\left\{\begin{array}{l}
t_{n+1}=t_{n}+\frac{\Delta \tau_{k}}{\bar{n}(k)}, \quad \text { where } \\
\bar{n}(k):=\max \left\{\left\lceil\frac{\left|\Delta z_{k}^{m}\right|\left\|f^{\prime}\right\|_{\infty}}{C_{\mathrm{CFL}} \Delta x}\right\rceil, 1\right\} \quad \text { for } k=0,1,2, \ldots, m-1 .
\end{array}\right.
$$

Here, the constant $C_{\mathrm{CFL}}>0$ depends on the scheme used. We refer to (3.14 as the local CFL condition. The next theorem shows that adaptive timesteps can improve the efficiency of the numerical methods.

Theorem 3.4. Let $u \in C\left([0, T] ; L^{1}(\mathbb{R})\right)$ denote the unique pathwise entropy solution of 1.5) for given $u_{0} \in\left(L^{1} \cap B V\right)(\mathbb{R})$ with Leb $\left(\operatorname{supp}\left(u_{0}\right)\right)>0, f \in C^{2}(\mathbb{R})$ with $\left\|f^{\prime}\right\|_{\infty}>0$, and $z \in C_{0}^{0, \alpha}([0, T])$ with $\alpha \in(0,1]$. For any $m \geq 2$, let $\left\{\tau_{j}\right\}_{j=0}^{m} \subset[0, T]$ denote the uniform mesh with step size $\Delta \tau=T / m$ and assume the computational cost of generating the interpolant $z^{m}=\mathcal{I}^{m}[z]$ is $\Theta\left(m^{\beta}\right)$ for some $\beta \geq 1$, and that there exists an $\check{m} \geq 2$ and $c>0$ such that

$$
\left|z^{m}\right|_{\mathrm{BV}([0, T])}>c m^{(1-\alpha) / 3} \quad \forall m \geq \check{m} .
$$


Furthermore, let $U$ denote a numerical solution of the method in Section 3.1 satisfying the local CFL condition (3.14) and assume that the spatial support of $U([0, T])$ is covered by an interval $\left[a_{m}, b_{m}\right] \subset \mathbb{R}$ that satisfies

$$
c_{1} \leq b_{m}-a_{m} \leq c_{2}(1+N \Delta x),
$$

for some $c_{1}, c_{2}>0$, cf. 3.10 . Then, the optimal balance of the resolution parameters for minimizing computational cost versus accuracy is

$$
\Delta x=\Theta\left(\frac{\Delta \tau^{\alpha}}{\left|z^{m}\right|_{B V([0, T])}^{2}}\right) \quad \text { and } \quad N=\sum_{k=0}^{m-1} \bar{n}(k)=\Theta\left(\frac{\left|z^{m}\right|_{B V([0, T])}^{3}}{\Delta \tau^{\alpha}}\right),
$$

and

$$
\|u(T)-U(T)\|_{1}=\mathcal{O}\left(m^{-\alpha / 2}\right)
$$

is achieved at the computational cost

$$
\hat{c}_{1}\left(\left|z^{m}\right|_{B V([0, T])}^{5} m^{2 \alpha}+m^{\beta}\right) \leq \operatorname{Cost}(U) \leq \hat{c}_{2}\left(\left|z^{m}\right|_{B V([0, T])}^{6} m^{2 \alpha}+m^{\beta}\right),
$$

for some $\hat{c}_{1}, \hat{c}_{2}>0$.

Remark 3.3. If $\left|z^{m}\right|_{B V([0, T])}=\Theta\left(\left\|\dot{z}^{m}\right\|_{\infty}\right)$, then the computational cost results in Theorems 3.3 and 3.4 are, up to constants, equivalent.

Proof. The local CFL condition (3.14 implies that all timesteps $\Delta t_{n}$ belonging to the same interpolation interval $\left[\tau_{j}, \tau_{j+1}\right]$ are of equal size and

$$
\Delta t_{n} \leq C_{\mathrm{CFL}} \frac{\Delta \tau \Delta x}{\left|\Delta z_{j}^{m}\right|\left\|f^{\prime}\right\|_{\infty}},
$$

for any $j \in\{0,1, \ldots, m-1\}$ and all $n \in\{0,1, \ldots, N\}$ such that $t_{n} \in\left[\tau_{j}, \tau_{j+1}\right)$. By (3.16) and the proof of Kuznetsov's lemma (see e.g. [51, with the flux $f$ replaced by $\dot{z}_{j}^{m} f$ ), the numerical error from one interpolation interval can be bounded by

$$
\begin{aligned}
\left\|u^{m}\left(\tau_{j+1}\right)-U\left(\tau_{j+1}\right)\right\|_{1} \leq & \| \begin{array}{l}
u^{m}\left(\tau_{j}\right)-U\left(\tau_{j}\right) \|_{1} \\
\end{array} \\
& +C\left|\dot{z}_{j}^{m}\right| \sum_{n \in\left\{0 \leq k \leq N \mid t_{k} \in\left[\tau_{j}, \tau_{j+1}\right)\right\}} \Delta t_{n}\left(\sqrt{\Delta t_{n}}+\sqrt{\Delta x}\right) \\
\leq & \left\|u^{m}\left(\tau_{j}\right)-U\left(\tau_{j}\right)\right\|_{1}+C\left(\left|\Delta z_{j}^{m}\right|+\Delta \tau\right) \sqrt{\Delta x}
\end{aligned}
$$

for some $C>0$ that depends on $\left\|f^{\prime}\right\|_{\infty},\left|u_{0}\right|_{B V(\mathbb{R})}$. Consequently, the error over $[0, T]$ is bounded by

$$
\left\|u^{m}(T)-U(T)\right\|_{1} \leq\left\|u_{0}-U(0)\right\|_{1}+C\left(\left|z^{m}\right|_{B V([0, T])}+T\right) \sqrt{\Delta x} .
$$

By a similar argument as in the proof of the preceding theorem, we conclude that

$$
\|u(T)-U(T)\|_{1}=\mathcal{O}\left(\Delta \tau^{\alpha / 2}+\left|z^{m}\right|_{B V([0, T])} \sqrt{\Delta x}\right),
$$

where, by 3.15 , we have used that $\left|z^{m}\right|_{B V([0, T])}+T=\mathcal{O}\left(\left|z^{m}\right|_{B V([0, T])}\right)$. The error contribution of the resolution parameters are balanced by

$$
\Delta x=\Theta\left(\frac{\Delta \tau^{\alpha}}{\left|z^{m}\right|_{B V([0, T])}^{2}}\right) .
$$

Assume that $m \geq \check{m}$. By 3.14 ,

$$
\frac{\left\|f^{\prime}\right\|_{\infty}}{C_{\mathrm{CFL}}} \frac{\left|\Delta z_{k}^{m}\right|}{\Delta x} \leq \bar{n}(k) \leq \frac{\left\|f^{\prime}\right\|_{\infty}}{C_{\mathrm{CFL}}} \frac{\left|\Delta z_{k}^{m}\right|}{\Delta x}+1 \quad \forall k \in\{0,1, \ldots, m-1\},
$$


and 3.15 implies that

$$
\begin{aligned}
N=\sum_{k=0}^{m-1} \bar{n}(k) & \leq \sum_{k=0}^{m-1}\left(\frac{\left\|f^{\prime}\right\|_{\infty}}{C_{\mathrm{CFL}}} \frac{\left|\Delta z_{k}^{m}\right|}{\Delta x}+1\right) \\
& \leq \frac{\left\|f^{\prime}\right\|_{\infty}}{C_{\mathrm{CFL}}} \frac{\left|z^{m}\right|_{B V([0, T])}^{3}}{\Delta \tau^{\alpha}}+m \\
& =\mathcal{O}\left(\frac{\left|z^{m}\right|_{B V([0, T])}^{3}}{\Delta \tau^{\alpha}}\right) .
\end{aligned}
$$

From 3.17 and 3.18 we conclude that

$$
N=\Theta\left(\frac{\left|z^{m}\right|_{B V([0, T])}^{3}}{\Delta \tau^{\alpha}}\right) .
$$

The computational cost of $U(T)$ is the sum of $\mathcal{O}\left(\mathrm{m}^{\beta}\right)$, for generating the piecewise linear interpolant $z^{m}$, and

$$
\Theta\left(N \times \frac{b_{m}-a_{m}}{\Delta x}\right)
$$

for solving $U$ over $[0, T] \times\left[a_{m}, b_{m}\right]$.

Remark 3.4. In his recent work 74 Seeger proposes a general class of monotone schemes and develops an accompanying error estimation theory for Hamilton-Jacobi equations of the form $d u=H(D u) \circ d W$, where $W=\left(W^{1}, \ldots, W^{k}\right)$ is a continuous path and $H=\left(H^{1}, \ldots, H^{k}\right)$ is a given nonlinear Hamiltonian. Besides that, a convergence theory is provided for a more general class of equations containing also a nonlinear degenerate elliptic operator $F\left(D^{2} u, D u, u\right)$. Similar to our approach, the approximation schemes of Seeger replace $W$ by a regular path $W_{h}$ defined over a partition $\mathcal{P}_{h}=\left\{0=t_{0}<t_{1}<\cdots<t_{N}=T\right\}$ with $\left|\mathcal{P}_{h}\right| \rightarrow 0$ as $h \rightarrow 0$. Also the temporal discretization of $d u$ utilizes the partition $\mathcal{P}_{h}$. The approximating path and the corresponding partition are chosen carefully to ensure that $\frac{\left|W_{h}\left(t_{n+1}\right)-W_{h}\left(t_{n}\right)\right|}{h} \lesssim 1$, where $t_{n}$ and $t_{n+1}$ are two consecutive points in the partition $\mathcal{P}_{h}$, and $h$ is the spatial discretization parameter of the schemes. In 74 the theory of viscosity solutions is used to derive new $L^{\infty}$ error estimates. The $L^{\infty}$ convergence rate depends, as in our case, on the exponent of the Hölder continuity of the driving path, and it is generally higher than the $L^{1}$ convergence rate we would obtain by Theorem 3.3 when using the same scalar driving path. As our problem has less spatial regularity and is likely to be more sensitive to perturbations in the driving path than Seeger's (compare $[2.3$ to [74, inequality (5.7)]), is it is not clear what conclusions may be drawn from this rate comparison.

3.4. Numerical examples. To simplify the spatial discretization in our numerical tests, we consider the following version of 1.5 with periodic boundary conditions:

$$
\begin{aligned}
& d u+\partial_{x} f(u) \circ d z=0 \quad \text { in }(0, T] \times \mathbb{T}, \\
& u(0, \cdot)=u_{0} \in\left(L^{1} \cap B V\right)(\mathbb{T}) .
\end{aligned}
$$

Well-posedness and stability results for 3.19 can be derived by a simple extension of 62. Lemma 3.1, and the numerical framework of Section 3.1 extend trivially to the periodic setting using the solution operator

$$
\mathcal{S}_{\mathbb{T}}(\cdot) \cdot: \mathbb{R} \times[0, \infty) \times\left(L^{1} \cap B V\right)(\mathbb{T}) \rightarrow\left(L^{1} \cap B V\right)(\mathbb{T}),
$$

where, for $c \in \mathbb{R}, s \geq 0$ and $v \in\left(L^{1} \cap B V\right)(\mathbb{T}), \mathcal{S}_{\mathbb{T}}^{c}(s) v$ denotes the solution at time $t=s$ of the conservation law

$$
\partial_{t} u+c \partial_{x} f(u)=0 \quad \text { in }(0, \infty) \times \mathbb{T}, \quad u(0, \cdot)=v .
$$


All problems are solved with the adaptive timestep method of Theorem 3.4 with free/varying resolution parameter $m \geq 2$, linked parameters

$$
\Delta \tau=m^{-1}, \quad \Delta x=\frac{1}{\left\lceil m^{\alpha}\left|z^{m}\right|_{B V([0, T])}^{2}\right\rceil},
$$

and $\Delta t_{n}$ determined by 3.14 with $C_{\mathrm{CFL}}=1$, for both the Lax-Friedrichs and the Engquist--Osher scheme.

Example 3.1. We consider 3.19 with $T=1, f(u)=u^{2} / 2, u_{0}(x)=\mathbf{1}_{[1 / 4,3 / 4]}(x)$, and the zigzag path $z \in C_{0}^{0,1}([0,1])$ generated by piecewise linear interpolation of the points $\left\{\left(t_{i}, z\left(t_{i}\right)\right)\right\}_{i=0}^{8}$ with $t_{i}=i / 8$ and

$$
z\left(t_{i}\right)= \begin{cases}0 & i \text { odd } \\ \frac{(-1)^{i / 2}}{4} & i \text { even }\end{cases}
$$

Thanks to Lemma 3.1, the solution can be represented as

$$
u(t, \cdot)= \begin{cases}\mathbf{1}_{[3 / 8,5 / 8]}(x) & \text { if } t=0, \\ \mathcal{S}_{\mathbb{T}}^{2}(t) u_{0} & \text { if } t \in\left(t_{0}, t_{1}\right], \\ \mathcal{S}_{\mathbb{T}}^{-2}\left(t-t_{1}\right) \mathcal{S}_{\mathbb{T}}^{2}(1 / 8) u_{0} & \text { if } t \in\left(t_{1}, t_{2}\right], \\ \vdots & \\ \mathcal{S}_{\mathbb{T}}^{2}\left(t-t_{7}\right) \mathcal{S}_{\mathbb{T}}^{-2}(1 / 8) \ldots \mathcal{S}_{\mathbb{T}}^{2}(1 / 8) u_{0} & \text { if } t \in\left(t_{7}, 1\right] .\end{cases}
$$

Moreover, using the method of characteristics and the auxiliary function $\psi: A \rightarrow \mathbb{R}$ defined on the domain $A=\left\{(a, b, c, d, x) \in \mathbb{T}^{5} \mid a \leq b \leq c \leq d\right.$ and $\left.a<d\right\}$ by

$$
\psi(a, b, c, d, x):= \begin{cases}0 & 0 \leq x \leq a \\ (x-a) /(b-a) & a<x<b \\ 1 & b \leq x \leq c \\ (d-x) /(d-c) & c<x<d \\ 0 & d \leq x<1\end{cases}
$$

we obtain the exact solution

$$
u(t, x)= \begin{cases}\psi(3 / 8,3 / 8+2 t, 5 / 8+t, 5 / 8+t, x) & t \in[0,1 / 8], \\ \psi(3 / 8,5 / 8-2(t-1 / 8), 6 / 8-2(t-1 / 8), 6 / 8, x) & t \in(1 / 8,1 / 4], \\ \psi(3 / 8-(t-1 / 4), 3 / 8-(t-1 / 4), 4 / 8-2(t-1 / 4), 6 / 8, x) & t \in(1 / 4,3 / 8], \\ \psi(2 / 8,2 / 8+2(t-3 / 8), 2 / 8+2(t-3 / 8), 6 / 8, x) & t \in(3 / 8,1 / 2], \\ \psi(2 / 8,4 / 8+2(t-1 / 2), 4 / 8+2(t-1 / 2), 6 / 8, x) & t \in(1 / 2,5 / 8], \\ \psi(2 / 8,6 / 8-2(t-5 / 8), 6 / 8-2(t-5 / 8), 6 / 8, x) & t \in(5 / 8,3 / 4], \\ \psi(2 / 8,4 / 8-2(t-3 / 4), 4 / 8-2(t-3 / 4), 6 / 8, x) & t \in(3 / 4,7 / 8], \\ \psi(2 / 8,2 / 8+2(t-7 / 8), 2 / 8+2(t-7 / 8), 6 / 8, x) & t \in(7 / 8,1] .\end{cases}
$$

Figure 1 shows snapshots of the exact solution of $u$ for the above problem, and corresponding numerical solutions $U$ computed with the Lax-Friedrichs and the Engquist-Osher scheme. The free resolution parameter is set to $m=2^{3}$ in first time series and $m=2^{6}$ in the second one. Since $\alpha=1,\left\|f^{\prime}\right\|_{\infty}=1$ and $\left|z^{m}\right|_{\mathrm{BV}([0,1])}=2$, equations (3.14) and (3.20) yield $\Delta x=2^{-5}, N=2^{6}$ when $m=2^{3}$ and $\Delta x=2^{-8}$, $N=2^{9}$ when $m=2^{6}$. As is to be expected from Theorem 3.4 the numerical solutions converge towards the exact solution as $m$ increases, and the EngquistOsher approximations converges faster than the Lax-Friedrichs approximations. 
Observe further from 3.22 and Figure 1 that for any $s, t \in\left[t_{3}, 1\right]$ such that $z(s)=z(t)$, it holds that $u(s)=u(t)$. In the next section we will explain this property by showing that certain "oscillating cancellations" in $z$ lead to corresponding cancellations in the solution $u$.
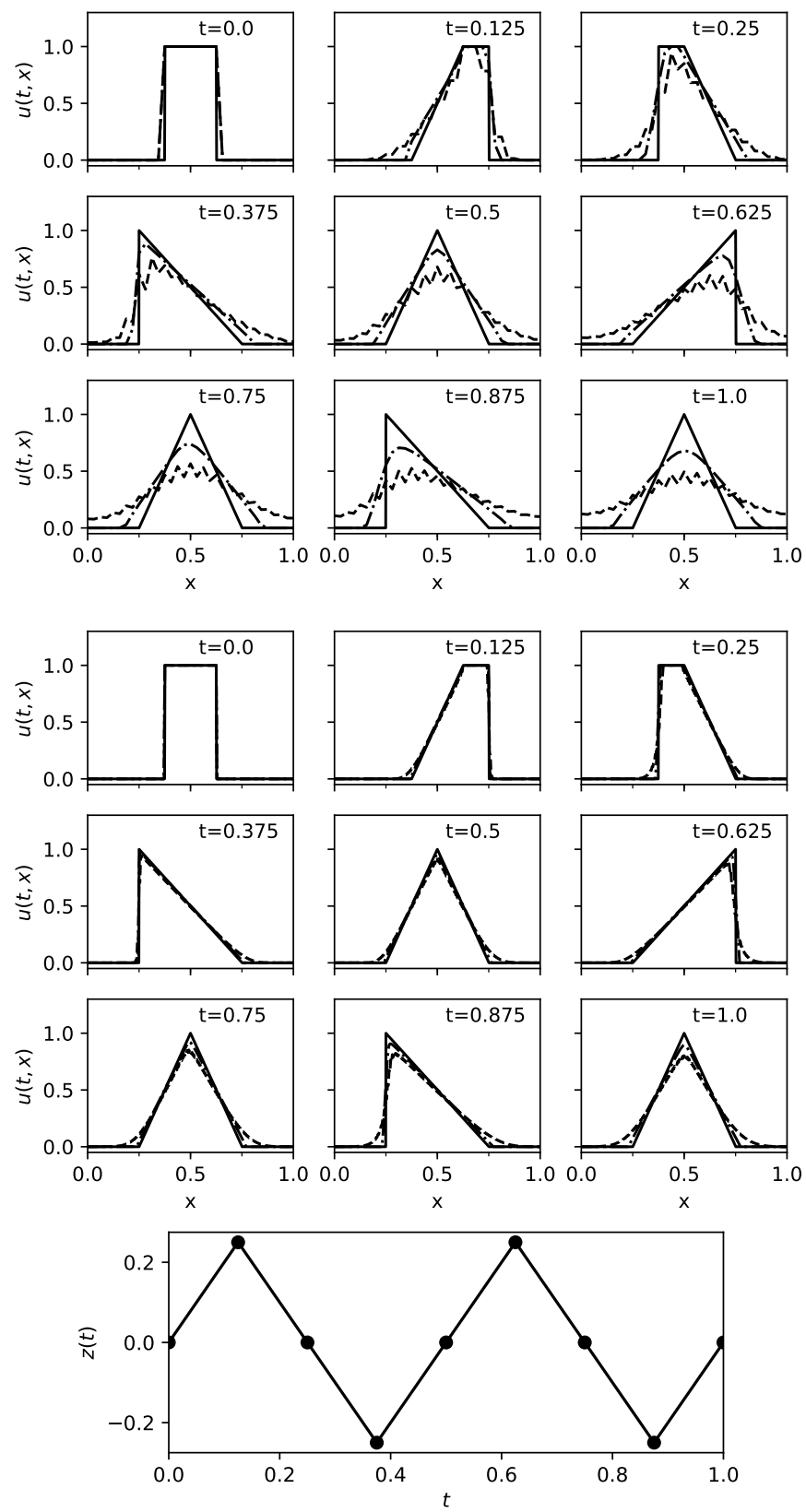

Figure 1. Top and middle: Time series snapshots of the exact (solid line), the Lax-Friedrichs (dashed line) and the EngquistOsher (dash-dotted line) solutions of Example 3.1 with flux $f(u)=$ $u^{2} / 2$ and free resolution parameter $m=2^{3}$ (top $3 \times 3$ subfigure) and $m=2^{6}$ (middle $3 \times 3$ subfigure). Bottom: The zigzag driving path $z$. The dotted points correspond to the value of $z$ at the respective time series snapshots. 
Figure 2 shows snapshots of numerical solutions of the above problem with the only difference being that the flux function here is $f(u)=u^{3} / 3$. The free resolution parameter is set to $m=2^{3}$ for the first time series and $m=2^{6}$ for the second one, and an approximate reference solution is computed at resolution $m=2^{12}$ using the Engquist-Osher scheme. We observe that the numerical solutions converge towards the reference solution, and a similar cancellation property as that for $f(u)=u^{2} / 2$ seems to hold at the snapshot times $t_{i}, t_{j} \geq t_{3}$ displayed here as well.
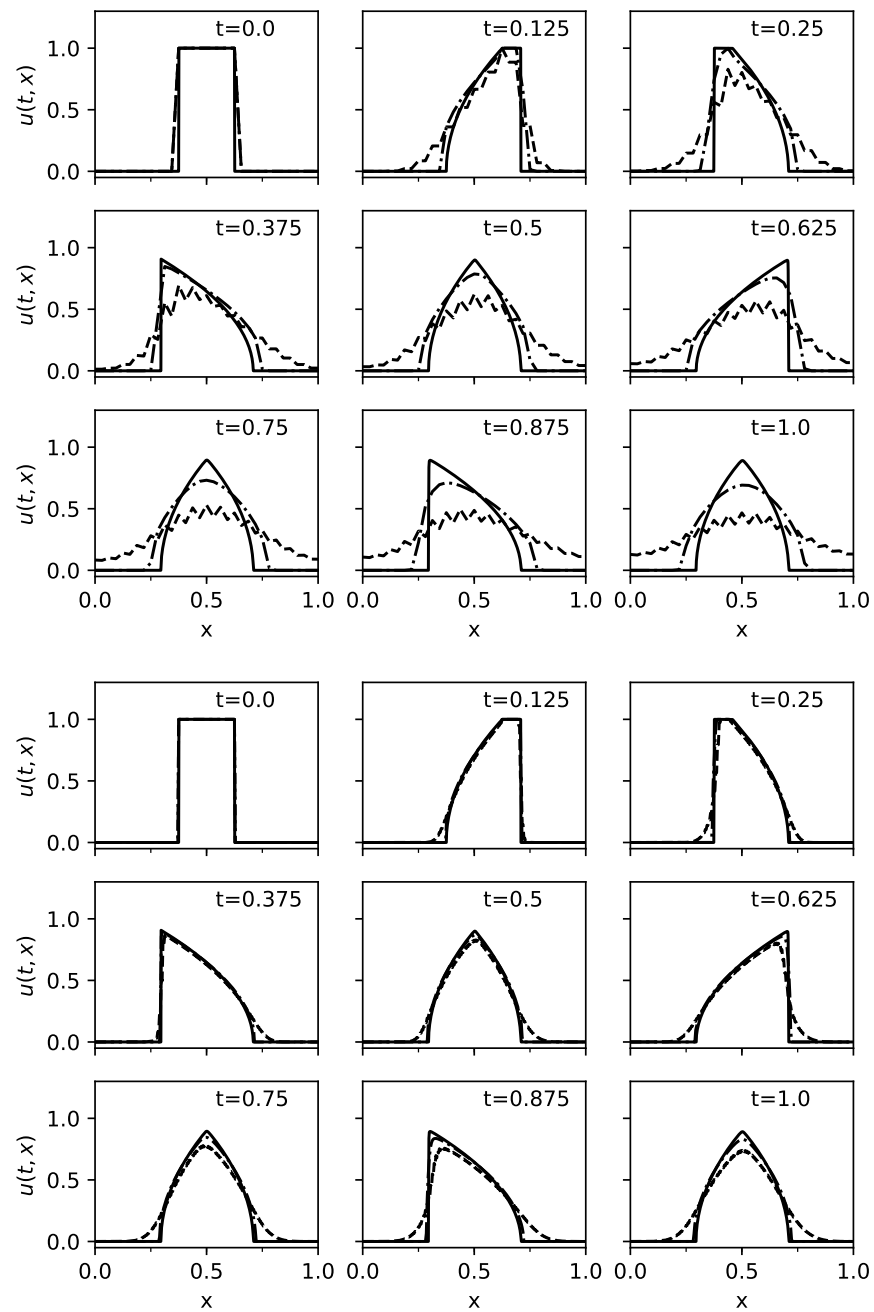

Figure 2. Example 3.1 with flux $f(u)=u^{3} / 3$. Approximated reference solution (solid), Lax-Friedrichs (dashed) and EngquistOsher (dash-dotted). The free resolution parameter is $m=2^{3}$ in the top $3 \times 3$ subfigure, and $m=2^{6}$ in the bottom one.

Example 3.2. We study the problem 3.19 with $T=1$, initial function $u_{0}(x)=$ $\mathbf{1}_{[3 / 8,5 / 8]}(x)$, and flux $f(u)=u^{2} / 2$. As driving path $z \in C_{0}^{0, \alpha}([0,1])$ we consider realizations of a fractional Brownian motion (fBM) with Hurst index $11=1 / 4,1 / 2$ and 3/4. We refer to [59] for details on fBM and the circulant embedding algorithm,

\footnotetext{
${ }^{1}$ To be precise, a sample path of an fBM with Hurst index $\alpha \in(0,1)$ is almost surely $(\alpha-\delta)$ Hölder continuous for all $\delta>0$, but it is almost surely not $\alpha$-Hölder continuous.
} 
which we use here to generate realizations on a uniformly spaced mesh $\left\{\tau_{k}\right\}_{k=0}^{m}$. The cost of generating $z^{m}$ with this method is $\mathcal{O}(m \log (m))$.
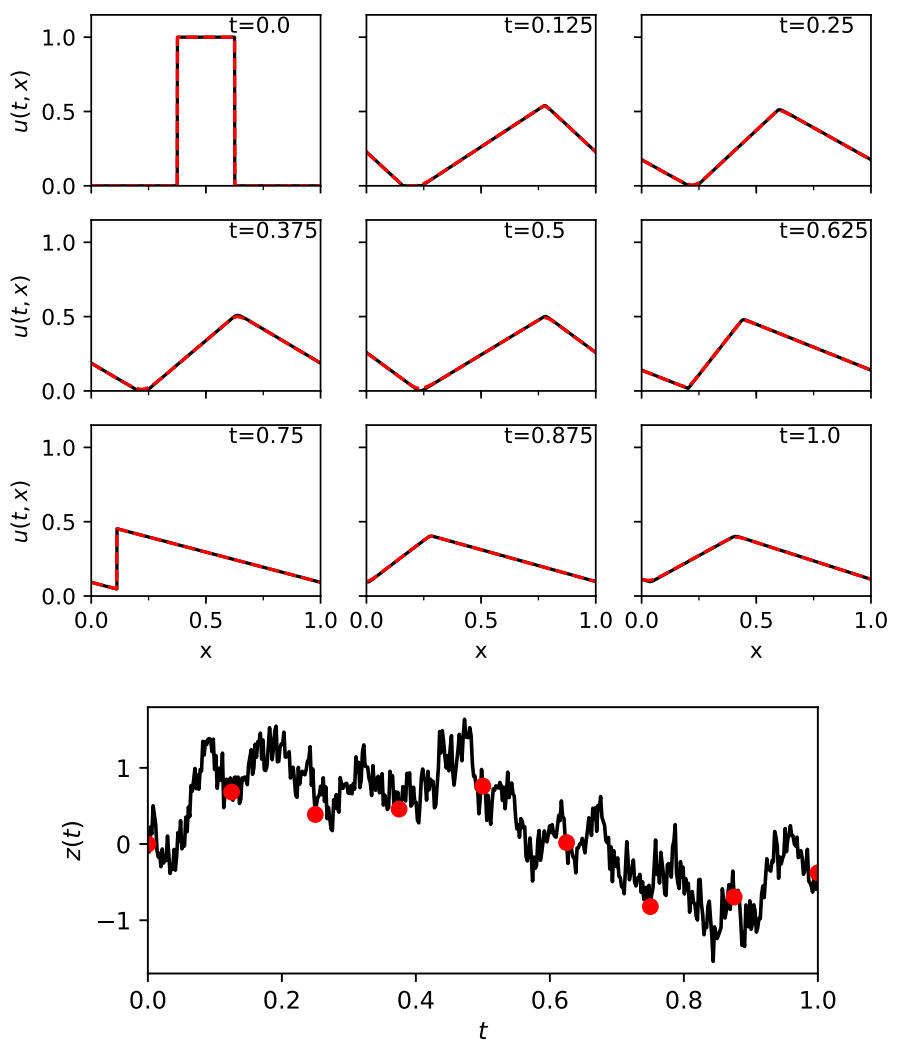

Figure 3. Top: Time series of numerical solutions with $\alpha=0.25$ for Example 3.2. Lax-Friedrichs (red dashed line) and EngquistOsher (black line). Bottom: The driving path $z$. Red points correspond to the value of $z$ at the respective time series snapshots.

Figures 3, 4, and 5 show time series of "adaptive timestep" numerical solutions for the respective Hurst indices $\alpha=0.25,0.5$ and 0.75 . The free resolution parameter is set to $m=2^{8}$. In Figure 6 we compare the final time numerical solutions $U(1)$ computed with the respective resolutions $m=2^{6}$ and $m=2^{8}$, and with an approximate reference solution computed with resolution $m=2^{10}$ using the more accurate numerical method developed in Section 5. A link to the other resolution parameters is obtained through $(3.20$ and the following property for fBMs: $\mathbb{E}\left[\left|z^{m}\right|_{\mathrm{BV}([0,1])}\right]=\Theta\left(m^{1-\alpha}\right)$. At resolution $m=2^{8}$, for instance, a typical realization of an fBM sample path yields $\Delta x=2^{-10}$ and $N=2^{12}$ for $\alpha=3 / 4 ; \Delta x=2^{-12}$ and $N=2^{16}$ for $\alpha=1 / 2$; and $\Delta x=2^{-14}$ and $N=2^{20}$ for $\alpha=1 / 4$. We observe that the Engquist-Osher scheme introduces less artificial diffusion and therefore produces more accurate approximations than the the Lax-Friedrichs scheme. (Note that solutions for different values of $\alpha$ are not directly comparable, not least since they are generated from independent fBM sample paths.)

Figure 7 shows the final time approximation error $\|U(1)-u(1)\|_{1}$ as a function of the resolution parameter $m$ for both numerical schemes. The error is averaged over $10 \mathrm{fBM}$ realizations for each of the considered Hurst indices. The convergence 

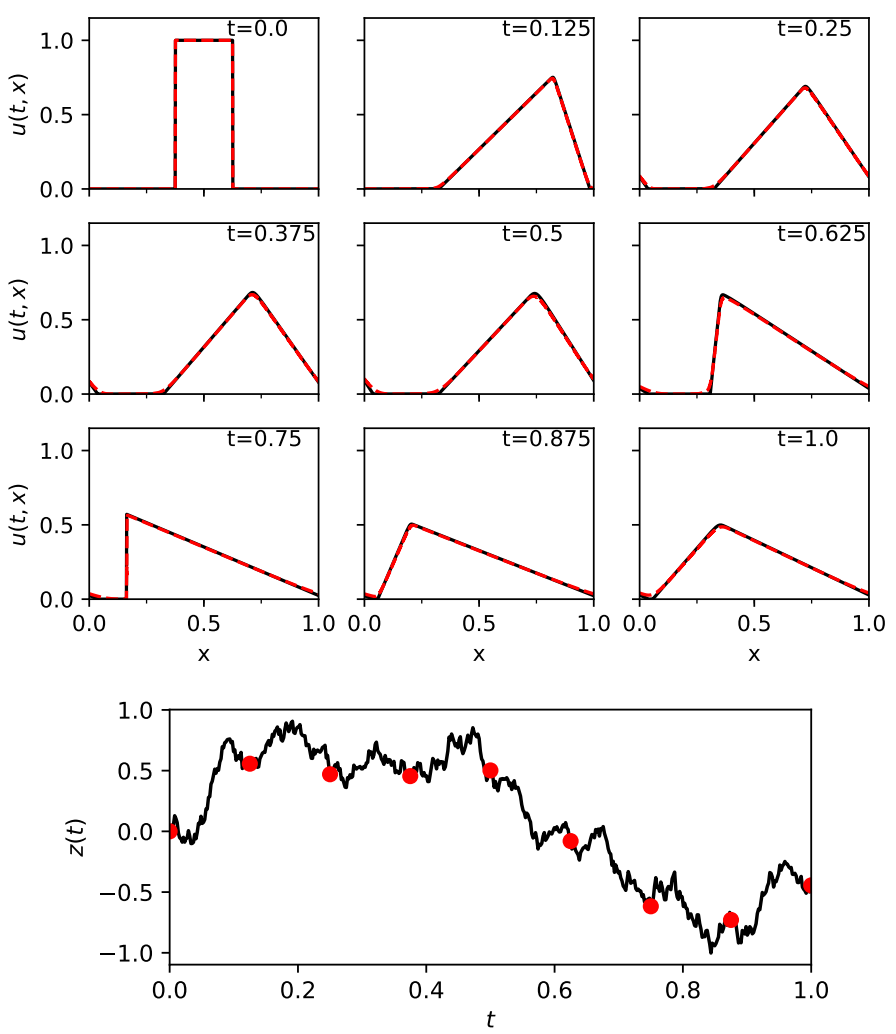

Figure 4. Top: Time series of numerical solutions with $\alpha=0.5$ for Example 3.2. Lax-Friedrichs (red dashed line) and EngquistOsher (black line). Bottom: The driving path $z$. Red points correspond to the value of $z$ at the respective time series snapshots.

rate decreases as $\alpha$ (and thus the regularity of $z$ ) decreases, but it is consistently orders of magnitude faster than Theorem 3.4 s possible worst case, $\mathcal{O}\left(m^{-\alpha / 2}\right)$.

\section{4. "Oscillatory CANCEllations"}

The numerical experiments in Example 3.2 indicate that the convergence rates obtained in Theorems 3.3 and 3.4 might not be sharp. In view of the more precise adaptive timestep error analysis of the latter theorem where the factor $\left|z^{m}\right|_{B V([0, T])}$ enters, one might suspect that the error bounds could be improved if one were able to identify "driving path oscillations" resulting in "cancellations" in the flux term $\dot{z}^{m} \partial_{x} f(u)$. In this section we show that for settings with $f \in C^{2}(\mathbb{R})$ strictly convex, the path $z^{m} \in I_{0}^{m}([0, T])$ can be replaced by a "simpler" path $y^{m} \in I_{0}^{m}([0, T])$ (with smaller total variation) that has the property that the solution of (2.1) with path $y^{m}$ coincides with the corresponding solution with path $z^{m}$ at final time $T$ (but not necessarily at earlier times).

In Section 4.1 we introduce some notation and give a definition of "oscillatory cancellations". Section 4.2 puts forward a mapping for removing "oscillatory cancellations" $\left(z^{m} \mapsto y^{m}\right)$ and culminates with Theorem 4.5 which asserts the equality of entropy solutions with the respective paths $z^{m}, y^{m}$ at final time $T$. In Section 4.3 we study properties of càdlàg paths with bounded total variation, cf. 4.21) and Theorem 4.10 The purpose of this is twofold. Firstly Theorem 4.10 provides an alternative stability result to that of Theorem 2.1. and, secondly, along the way a 

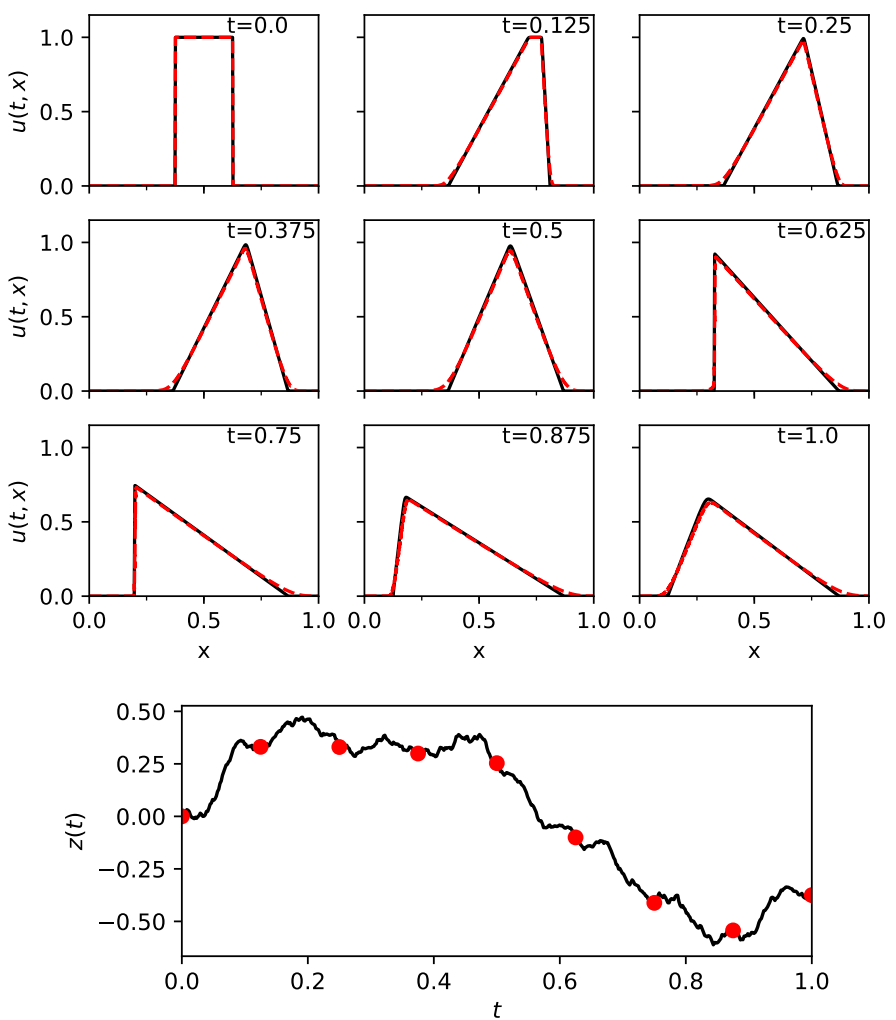

FiguRE 5. Top: Time series of numerical solutions with $\alpha=0.75$ for Example 3.2. Lax-Friedrichs (red dashed line) and EngquistOsher (black line). Bottom: The driving path $z$. Red points correspond to the value of $z$ at the respective time series snapshots.

constructive definition of $\lim _{m \rightarrow \infty} y^{m}$ is developed. The latter is useful in the study of qualitative properties of Wiener paths, cf. Lemma 5.3. An efficient numerical method making use of "oscillatory cancellations" is developed in Section 5.

4.1. Preliminaries. Recall that for $\kappa \in \mathbb{R}, s \geq 0$, and $v \in\left(L^{1} \cap B V\right)(\mathbb{R}), \mathcal{S}^{\kappa}(s) v$ denotes the solution at time $t=s$ of

$$
\partial_{t} u+\kappa \partial_{x} f(u)=0 \quad \text { in }(0, \infty) \times \mathbb{R}, \quad u(0, \cdot)=v,
$$

so the entropy solution at time $t=T$ of 2.1 with path $z^{m} \in I_{0}\left([0, T] ;\left\{\tau_{j}\right\}_{j=0}^{m}\right)$ can be expressed by

$$
u^{m}(T)=\mathcal{S}^{\dot{z}_{m-1}^{m}}(\Delta \tau) \mathcal{S}^{\dot{z}_{m-2}^{m}}(\Delta \tau) \ldots \mathcal{S}^{\dot{z}_{0}^{m}}(\Delta \tau) u_{0} .
$$

By a change of variables, the solution mapping can be simplified to only depend on the path increments.

Lemma 4.1. For any $\Delta \tau>0, \kappa \in \mathbb{R}$ and $v \in\left(L^{1} \cap B V\right)(\mathbb{R}), \mathcal{S}^{\kappa}(\Delta \tau) v$ coincides with the entropy solution at time $t=|\kappa| \Delta \tau$ of

$$
\partial_{t} \tilde{u}+\operatorname{sign}(\kappa) \partial_{x} f(\tilde{u})=0 \quad \text { in }(0, \infty) \times \mathbb{R}, \quad \tilde{u}(0)=v .
$$

Proof. For $\kappa=0$ the result trivially holds as $\mathcal{S}^{0}(\cdot)=I$. Otherwise, when $|\kappa|>0$, we verify the result by showing that

$$
\bar{u}(t, x):=\tilde{u}(t|\kappa|, x)
$$



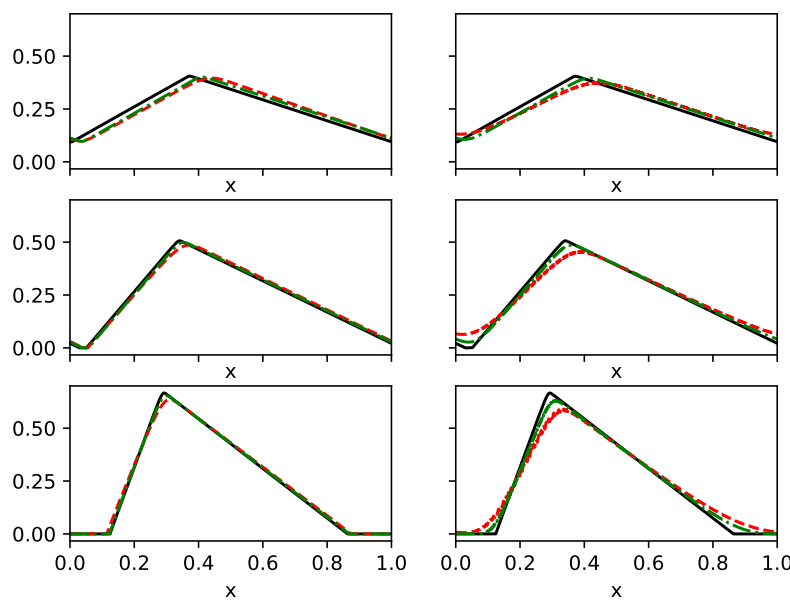

Figure 6. Final time solutions for Example 3.2, Reference solution (black line), numerical solutions at resolutions $m=2^{6}$ (red dashed line) and $m=2^{8}$ (green dash-dot line). Left column: Engquist-Osher scheme, Hurst index $\alpha=0.25,0.5,0.75$ from top to bottom, respectively. Right column: Lax-Friedrichs scheme, Hurst index $\alpha=0.25,0.5,0.75$ from top to bottom, respectively.

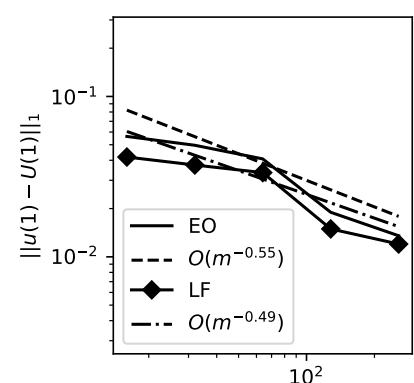

$\mathrm{m}$

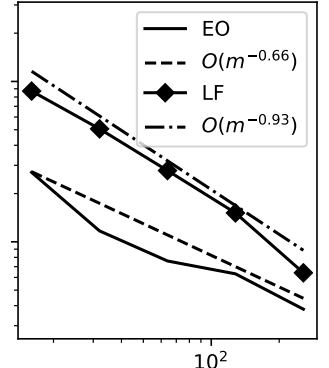

$\mathrm{m}$

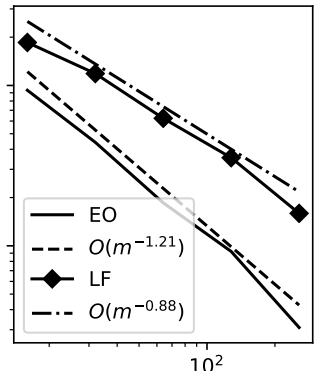

$\mathrm{m}$

FiguRE 7. Final time approximation error for Example 3.2. Hurst indices $\alpha=0.25,0.5,0.75$ from left to right, respectively. The abbreviations EO and LF denote respectively the Engquist-Osher and Lax-Friedrichs schemes.

is an entropy solution of

$$
\partial_{t} \bar{u}+\kappa \partial_{x} f(\bar{u})=0 \quad \text { in }(0, \infty) \times \mathbb{R}, \quad \bar{u}(0)=v .
$$

Let $\phi \in C_{0}^{\infty}(\mathbb{R} \times \mathbb{R})$ be an arbitrary nonnegative test function and set

$$
\bar{\phi}(t, x):=\phi(t|\kappa|, x) .
$$

By construction, for all $c \in \mathbb{R}$, we have

$$
\begin{gathered}
\int_{0}^{|\kappa| \Delta \tau} \int_{\mathbb{R}}|\tilde{u}-c| \partial_{t} \phi+\operatorname{sign}(\tilde{u}-c) \operatorname{sign}(\kappa)(f(\tilde{u})-f(c)) \partial_{x} \phi \mathrm{d} x \mathrm{~d} t \\
\quad+\int_{\mathbb{R}}|\tilde{u}(0, x)-c| \phi(0, x)-|\tilde{u}(|\kappa| \Delta \tau, x)-c| \phi(|\kappa| \Delta \tau, x) \mathrm{d} x \geq 0 .
\end{gathered}
$$


Making the change of variables $\bar{t}=t /|\kappa|$, we arrive at

$$
\begin{aligned}
& \int_{0}^{\Delta \tau} \int_{\mathbb{R}}|\bar{u}-c|\left(\partial_{t} \phi\right)(\bar{t}|\kappa|, x) \\
& \quad+\operatorname{sign}(\bar{u}-c) \kappa(f(\bar{u})-f(c))\left(\partial_{x} \phi\right)(\bar{t}|\kappa|, x) \mathrm{d} x \mathrm{~d} \bar{t} \\
& \quad+\int_{\mathbb{R}}|\bar{u}(0, x)-c| \bar{\phi}(0, x)-|\bar{u}(\Delta \tau, x)-c| \bar{\phi}(\Delta \tau, x) \mathrm{d} x \geq 0 .
\end{aligned}
$$

Noting that

it follows that

$$
\partial_{t} \phi(\bar{t}|\kappa|, x)=\partial_{\bar{t}} \phi(\bar{t}|\kappa|, x)|\kappa|^{-1}
$$

$$
\begin{aligned}
& \int_{0}^{\Delta \tau} \int_{\mathbb{R}}|\bar{u}-c| \partial_{\bar{t}} \bar{\phi}+\operatorname{sign}(\bar{u}-c) \kappa(f(\bar{u})-f(c)) \partial_{x} \bar{\phi} \mathrm{d} x \mathrm{~d} \bar{t} \\
& \quad+\int_{\mathbb{R}}|\bar{u}(0, x)-c| \bar{\phi}(0, x)-|\bar{u}(\Delta \tau, x)-c| \bar{\phi}(\Delta \tau, x) \mathrm{d} x \geq 0 .
\end{aligned}
$$

In view of (4.3) and the invertibility of the mapping $t \mapsto t|\kappa|$, the above inequality holds for arbitrary nonnegative $\bar{\phi} \in C_{0}^{\infty}(\mathbb{R} \times \mathbb{R})$.

Let $\overline{\mathcal{S}}(\cdot): \mathbb{R} \times\left(L^{1} \cap B V\right)(\mathbb{R}) \rightarrow\left(L^{1} \cap B V\right)(\mathbb{R})$ be the solution operator linked to

$$
\partial_{t} \tilde{u}+\operatorname{sign}(\Delta z) \partial_{x} f(\tilde{u})=0 \quad \text { in }(0, \infty) \times \mathbb{R}, \quad \tilde{u}(0)=v .
$$

That is, for $\Delta z \in \mathbb{R}$ and $v \in\left(L^{1} \cap B V\right)(\mathbb{R}), \overline{\mathcal{S}}(\Delta z) v$ denotes the Kružkov entropy solution at time $t=|\Delta z|$ of $(4.4)$.

Lemma 4.1 implies that for any $z^{m} \in I_{0}\left([0, T] ;\left\{\tau_{j}\right\}_{j=0}^{m}\right), k \in\{0,1, \ldots, m-1\}$, $s \in\left[0, \Delta \tau_{k}\right]$ and $v \in\left(L^{1} \cap B V\right)(\mathbb{R})$,

$$
\mathcal{S}^{\dot{z}_{k}^{m}}(s) v=\overline{\mathcal{S}}\left(z^{m}\left(\tau_{k}+s\right)-z^{m}\left(\tau_{k}\right)\right) v \text {. }
$$

In view of 4.5 and 3.3 , the solution of 2.1), for given $u_{0} \in\left(L^{1} \cap B V\right)(\mathbb{R})$, $f \in C^{2}(\mathbb{R})$ and driving path $z^{m} \in I_{0}^{m}([0, T])$, can be represented by

$$
u^{m}(t)= \begin{cases}\overline{\mathcal{S}}\left(z^{m}(t)-z^{m}(0)\right) u_{0} & \text { if } t \in\left[0, \tau_{1}\right], \\ \overline{\mathcal{S}}\left(z^{m}(t)-z^{m}\left(\tau_{1}\right)\right) \overline{\mathcal{S}}\left(\Delta z_{0}^{m}\right) u_{0} & \text { if } t \in\left(\tau_{1}, \tau_{2}\right], \\ \cdots & \\ \overline{\mathcal{S}}\left(z^{m}(t)-z^{m}\left(\tau_{m-1}\right)\right) \overline{\mathcal{S}}\left(\Delta z_{k-2}^{m}\right) \cdots \overline{\mathcal{S}}\left(\Delta z_{0}^{m}\right) u_{0} & \text { if } t \in\left(\tau_{m-1}, T\right] .\end{cases}
$$

To study how an entropy solution depends on the driving path, we introduce the notion "oscillatory cancellations".

Definition 4.1. For given $u_{0} \in\left(L^{1} \cap B V\right)(\mathbb{R}), f \in C^{2}(\mathbb{R})$ and $z^{m} \in I_{0}^{m}([0, T])$, we say there are "oscillatory cancellations" over an interval $\left[\tau_{k}, \tau\right] \subset[0, T]$, with $k \in\{0,1, \ldots, m-2\}$ and $\tau \in\left(\tau_{\ell}, \tau_{\ell+1}\right]$ for some $k<\ell \leq m-1$, if it holds that

$$
\begin{aligned}
u^{m}(\tau) & =\overline{\mathcal{S}}\left(z^{m}(\tau)-z^{m}\left(\tau_{\ell}\right)\right) \cdots \overline{\mathcal{S}}\left(\Delta z_{k}^{m}\right) u^{m}\left(\tau_{k}\right) \\
& =\overline{\mathcal{S}}\left(z^{m}(\tau)-z^{m}\left(\tau_{\ell}\right)+\sum_{j=k}^{\ell-1} \Delta z_{j}^{m}\right) u^{m}\left(\tau_{k}\right) \\
& =\overline{\mathcal{S}}\left(z^{m}(\tau)-z^{m}\left(\tau_{k}\right)\right) u^{m}\left(\tau_{k}\right) .
\end{aligned}
$$

Recall from [51, Theorem 2.15] that the solution operator $\overline{\mathcal{S}}$ fulfills the following properties for all $u, v \in\left(L^{1} \cap B V\right)(\mathbb{R})$ and $s, t \in \mathbb{R}$ :

$$
\begin{aligned}
\|\overline{\mathcal{S}}(s) u-\overline{\mathcal{S}}(s) v\|_{L^{1}(\mathbb{R})} & \leq\|u-v\|_{L^{1}(\mathbb{R})}, \\
\|\overline{\mathcal{S}}(s) u-\overline{\mathcal{S}}(t) u\|_{L^{1}(\mathbb{R})} & \leq\left\|f^{\prime}\right\|_{L^{\infty}}|u|_{\mathrm{BV}(\mathbb{R})}|t-s| \\
|\overline{\mathcal{S}}(s) u|_{\mathrm{BV}(\mathbb{R})} & \leq\left|u_{0}\right|_{\mathrm{BV}(\mathbb{R})} .
\end{aligned}
$$


The spatial regularity of entropy solutions turns out to be strongly linked to the running maximum/minimum functions of the driving path introduced below.

Definition 4.2. For any $z \in C_{0}([0, T])$, let

$$
M^{+}[z](t):=\max _{s \in[0, t]} z(s) \quad \text { and } \quad M^{-}[z](t):=\min _{s \in[0, t]} z(s) \quad \text { for } \quad t \in[0, T]
$$

denote the running $\max / \min$ functions of $z$.

See Figure 8 for an example illustrating the running $\max / \min$ functions.

To identify intervals with oscillatory cancellations we make use of the following regularity result:

Theorem 4.2. Let $u^{m} \in C_{0}\left([0, T] ; L^{1}(\mathbb{R})\right)$ denote the unique entropy solution of (2.1), for given initial data $u_{0} \in\left(L^{1} \cap B V\right)(\mathbb{R})$, strictly convex flux $f \in C^{2}(\mathbb{R})$ and driving path $z^{m} \in I_{0}^{m}([0, T])$. If for some $0 \leq s_{1}<s_{2} \leq T$,

$$
z^{m}(t) \in\left(M^{-}\left[z^{m}\right]\left(s_{1}\right), M^{+}\left[z^{m}\right]\left(s_{1}\right)\right) \quad \forall t \in\left(s_{1}, s_{2}\right),
$$

then for all $t \in\left(s_{1}, s_{2}\right)$, it holds that $u^{m}(t) \in \operatorname{Lip}(\mathbb{R})$ and

$$
\sup _{x \neq y}\left|\frac{u^{m}(t, y)-u^{m}(t, x)}{y-x}\right| \leq \frac{\left\|\left(f^{\prime \prime}\right)^{-1}\right\|_{L^{\infty}}}{\min \left\{z^{m}(t)-M^{-}\left[z^{m}\right]\left(s_{1}\right), M^{+}\left[z^{m}\right]\left(s_{1}\right)-z^{m}(t)\right\}},
$$

where (as usual) the $L^{\infty}$ norm is restricted to the interval $\left[-\left\|u_{0}\right\|_{\infty},\left\|u_{0}\right\|_{\infty}\right]$.

The theorem is a direct consequence of Lemma A.1, $f \in C^{2}(\mathbb{R})$, and the meanvalue theorem. We refer to the companion work [46] for an in-depth theoretical treatment of regularity and cancellation properties for (2.1.

Remark 4.1. A regularity result similar to Theorem 4.2 has been derived in 33 . There the authors investigate regularity properties of solutions to the stochastic Hamilton-Jacobi equation

$$
d v+\frac{1}{2}|D v|^{2} \circ d z=F\left(x, v, D v, D^{2} v\right) d t,
$$

where $z$ is a continuous path, and $F$ is a nonlinear function meeting the standard assumptions from the theory of viscosity solutions of fully nonlinear degenerate parabolic PDEs. An $L^{\infty}$-bound on the second derivative $D^{2} v$ is derived, and in the special case

$$
d u+\partial_{x}\left(\frac{u^{2}}{2}\right) \circ d z=0, \quad u=\partial_{x} v
$$

this estimate reduces to the Lipschitz $W^{1, \infty}$ bound

$$
\operatorname{ess} \sup _{x \neq y}\left|\frac{u(t, x)-u(t, y)}{x-y}\right| \leq \frac{1}{\min \left\{z(t)-M^{-}[z](t), M^{+}[z](t)-z(t)\right\}} .
$$

This regularity result is identical to Theorem 4.2 . But the regularity result in 33 is, in contrast to ours, restricted to the special case $f(u)=u^{2} / 2$ and the proofs differ: 33 uses semiconvexity preservation properties of Hamilton-Jacobi equations, while we (here and in [46) argue at the level of conservation laws and use the method of generalized characteristics.

For a given driving path $z^{m} \in I_{0}^{m}([0, T])$, the following lemma identifies intervals with "oscillatory cancellations".

Lemma 4.3. For an $m \geq 2$, let $u^{m} \in C_{0}\left([0, T] ; L^{1}(\mathbb{R})\right)$ denote the unique entropy solution of 2.1) for some initial data $u_{0} \in\left(L^{1} \cap B V\right)(\mathbb{R})$, strictly convex $f \in C^{2}(\mathbb{R})$ and driving noise $z^{m} \in I_{0}^{m}([0, T])$. Then property 4.7) holds over an interval $\left[\tau_{k}, \tau\right] \subset[0, T]$ with $k \in\{0,1, \ldots, m-2\}$ and $\tau \in\left(\tau_{\ell}, \tau_{\ell+1}\right]$ for some $k<\ell \leq m-1$, if at least one of the following conditions are met: 
(i) $\dot{z}^{m}(s+):=\lim _{\delta \downarrow 0} \dot{z}^{m}(s+\delta) \geq 0$ for all $s \in\left[\tau_{k}, \tau\right)$,

(ii) $\dot{z}^{m}(s+) \leq 0$ for all $s \in\left[\tau_{k}, \tau\right)$,

(iii) $z^{m}\left(\left[\tau_{k}, \tau\right]\right) \subset\left[M^{-}\left[z^{m}\right]\left(\tau_{k}\right), M^{+}\left[z^{m}\right]\left(\tau_{k}\right)\right]$.

Remark 4.2. Figure 8 exemplifies the running $\max / \min$ functions for a piecewise linear function $z^{10} \in I_{0}\left([0, T] ;\left\{\tau_{j}\right\}_{j=0}^{10}\right)$, where $\left[\tau_{5}, \tau_{7}\right]$ is of type $(\mathrm{i}),\left[\tau_{7}, \tau_{10}=T\right]$ is of type (ii), and $\left[\tau_{2}, \tau_{5}\right]$ and $\left[\tau_{7}, \tau\right]$ with $\tau=\tau_{9}+2 \Delta \tau / 3$ are of type (iii).

Proof. (i) \& (ii): The condition $\left.\dot{z}^{m}(\cdot+)\right|_{\left[\tau_{k}, \tau\right)} \geq 0$ implies that $\Delta z_{j}^{m} \geq 0$ for $j=k, k+1, \ldots, \ell-1$. By the definition of $\overline{\mathcal{S}}$, cf. 4.4.,

$$
\overline{\mathcal{S}}\left(z^{m}(\tau)-z^{m}\left(\tau_{\ell}\right)\right) \ldots \overline{\mathcal{S}}\left(\Delta z_{k}^{m}\right) u^{m}\left(\tau_{k}\right)
$$

is equal to the entropy solution at time $t=z^{m}(\tau)-z^{m}\left(\tau_{\ell}\right)+\sum_{j=k}^{\ell-1} \Delta z_{j}^{m}$ of

$$
\partial_{t} \tilde{u}+\operatorname{sign}\left(z^{m}(\tau)-z^{m}\left(\tau_{j}\right)\right) \partial_{x} f(\tilde{u})=0, \quad \tilde{u}(0)=u^{m}\left(\tau_{k}\right) .
$$

But, by definition, we also have that

$$
\overline{\mathcal{S}}\left(z^{m}(\tau)-z^{m}\left(\tau_{k}\right)\right) u^{m}\left(\tau_{k}\right)=\tilde{u}\left(z^{m}(\tau)-z^{m}\left(\tau_{k}\right)\right),
$$

and it is clear that (4.7) holds. Part (ii) follows by a similar argument.

(iii): We assume $M^{+}\left[z^{m}\right]\left(\tau_{k}\right)-M^{-}\left[z^{m}\right]\left(\tau_{k}\right)>0$, as otherwise $\left.\dot{z}^{m}(\cdot+)\right|_{\left[\tau_{k}, \tau\right)}=0$ and the cancellation property follows by (i) or (ii).

For a $\delta \in\left(0,\left(M^{+}\left[z^{m}\right]\left(\tau_{k}\right)-M^{-}\left[z^{m}\right]\left(\tau_{k}\right)\right) / 2\right)$ we define the approximation path $z^{m, \delta} \in I_{0}\left([0, T] ;\left\{\tau_{j}\right\}_{j=0}^{m}\right)$ by interpolating the values

$$
z^{m, \delta}\left(\tau_{j}\right):= \begin{cases}z^{m}\left(\tau_{j}\right)-\delta & \text { if } j \in\{k+1, \ldots, \ell\} \text { and } z^{m}\left(\tau_{j}\right)=M^{+}\left[z^{m}\right]\left(\tau_{k}\right) \\ z^{m}\left(\tau_{j}\right)+\delta & \text { if } j \in\{k+1, \ldots, \ell\} \text { and } z^{m}\left(\tau_{j}\right)=M^{-}\left[z^{m}\right]\left(\tau_{k}\right) \\ z^{m}\left(\tau_{j}\right) & \text { otherwise }\end{cases}
$$

over the set of interpolation points $\left\{\tau_{j}\right\}_{j=0}^{m}$. By construction, it then holds that

$$
\begin{gathered}
z^{m, \delta}(s) \in\left(M^{-}\left[z^{m}\right]\left(\tau_{k}\right), M^{+}\left[z^{m}\right]\left(\tau_{k}\right)\right), \quad \forall s \in\left(\tau_{k}, \tau\right), \\
\left|\Delta z_{j}^{m, \delta}-\Delta z_{j}^{m}\right| \leq 2 \delta, \quad \text { for all } \quad j \in\{k, k+1, \ldots, \ell-1\},
\end{gathered}
$$

where $\Delta z_{j}^{m, \delta}:=z^{m, \delta}\left(\tau_{j+1}\right)-z^{m, \delta}\left(\tau_{j}\right)$, and

$$
\left|z^{m, \delta}(\tau)-z^{m, \delta}\left(\tau_{\ell}\right)-\left(z^{m}(\tau)-z^{m}\left(\tau_{\ell}\right)\right)\right| \leq 2\left|z^{m, \delta}\left(\tau_{\ell}\right)-z^{m}\left(\tau_{\ell}\right)\right| \leq 2 \delta .
$$

Let $u^{m, \delta}$ denote the solution of $(2.1)$ with driving path $z^{m, \delta}$ and the same initial data $u_{0}$ and flux $f$ as the solution $u^{m}$. Since $\left.z^{m, \delta}\right|_{\left[0, \tau_{k}\right]}=\left.z^{m}\right|_{\left[0, \tau_{k}\right]}$, 4.4 implies

$$
u^{m, \delta}(t)= \begin{cases}u^{m}(t) & \text { if } t \leq \tau_{k}, \\ \overline{\mathcal{S}}\left(z^{m, \delta}(t)-z^{m}\left(\tau_{k}\right)\right) u^{m}\left(\tau_{k}\right) & \text { if } t \in\left(\tau_{k}, \tau_{k+1}\right] \\ \cdots & \\ \overline{\mathcal{S}}\left(z^{m, \delta}(t)-z^{m, \delta}\left(\tau_{\ell}\right)\right) \cdots \overline{\mathcal{S}}\left(\Delta z_{k}^{m, \delta}\right) u^{m}\left(\tau_{k}\right), & \text { if } t \in\left(\tau_{\ell}, \tau\right] .\end{cases}
$$

By Theorem 4.2 it holds that $u^{m, \delta}(s) \in \operatorname{Lip}(\mathbb{R})$ for all $s \in\left(\tau_{k}, \tau\right)$. Consequently, $\left.u^{m, \delta}\right|_{\left(\tau_{k}, \tau\right) \times \mathbb{R}}$ is a classical solution that is time invertible, as it can be obtained by 
the method of characteristics. By 4.9 , we further obtain that

$$
\begin{aligned}
\overline{\mathcal{S}}\left(z^{m, \delta}(\tau)-z^{m, \delta}\left(\tau_{\ell}\right)\right) \cdots \overline{\mathcal{S}}\left(\Delta z_{k}^{m, \delta}\right) u^{m}\left(\tau_{k}\right) \\
=\lim _{\epsilon \downarrow 0}\left[\overline{\mathcal{S}}\left(z^{m, \delta}(\tau)-z^{m, \delta}(\tau-\epsilon)\right) \overline{\mathcal{S}}\left(z^{m, \delta}(\tau-\epsilon)\right)-z^{m, \delta}\left(\tau_{\ell}\right)\right) \overline{\mathcal{S}}\left(\Delta z_{\ell-1}^{m, \delta}\right) \cdots \\
\left.\quad \overline{\mathcal{S}}\left(\Delta z_{k+1}^{m, \delta}\right) \overline{\mathcal{S}}\left(z^{m, \delta}\left(\tau_{k+1}\right)-z^{m, \delta}\left(\tau_{k}+\epsilon\right)\right) \overline{\mathcal{S}}\left(z^{m, \delta}\left(\tau_{k}+\epsilon\right)-z^{m, \delta}\left(\tau_{k}\right)\right) u^{m}\left(\tau_{k}\right)\right] \\
=\lim _{\epsilon \downarrow 0}\left[\overline{\mathcal{S}}\left(z^{m, \delta}(\tau)-z^{m, \delta}(\tau-\epsilon)\right) \overline{\mathcal{S}}\left(z^{m, \delta}(\tau-\epsilon)\right)-z^{m, \delta}\left(\tau_{k}+\epsilon\right)\right) \\
\left.\quad \overline{\mathcal{S}}\left(z^{m, \delta}\left(\tau_{k}+\epsilon\right)-z^{m, \delta}\left(\tau_{k}\right)\right) u^{m}\left(\tau_{k}\right)\right] \\
=\overline{\mathcal{S}}\left(z^{m, \delta}(\tau)-z^{m, \delta}\left(\tau_{k}\right)\right) u^{m}\left(\tau_{k}\right) .
\end{aligned}
$$

By 4.8, 4.9, 4.10, 4.12 and 4.13,

$$
\begin{aligned}
& \left\|\overline{\mathcal{S}}\left(z^{m}(\tau)-z^{m}\left(\tau_{\ell}\right)\right) \cdots \overline{\mathcal{S}}\left(\Delta z_{k}^{m}\right) u^{m}\left(\tau_{k}\right)-\overline{\mathcal{S}}\left(z^{m, \delta}(\tau)-z^{m, \delta}\left(\tau_{k}\right)\right) u^{m}\left(\tau_{k}\right)\right\|_{L^{1}} \\
& =\left\|\left[\overline{\mathcal{S}}\left(z^{m}(\tau)-z^{m}\left(\tau_{\ell}\right)\right) \cdots \overline{\mathcal{S}}\left(\Delta z_{k}^{m}\right)-\overline{\mathcal{S}}\left(z^{m, \delta}(\tau)-z^{m, \delta}\left(\tau_{\ell}\right)\right) \cdots \overline{\mathcal{S}}\left(\Delta z_{k}^{m, \delta}\right)\right] u^{m}\left(\tau_{k}\right)\right\|_{L^{1}} \\
& \leq\left\|\left[\overline{\mathcal{S}}\left(z^{m}(\tau)-z^{m}\left(\tau_{\ell}\right)\right)-\overline{\mathcal{S}}\left(z^{m, \delta}(\tau)-z^{m, \delta}\left(\tau_{\ell}\right)\right)\right] \overline{\mathcal{S}}\left(\Delta z_{\ell-1}^{m}\right) \cdots \overline{\mathcal{S}}\left(\Delta z_{k}^{m}\right) u^{m}\left(\tau_{k}\right)\right\|_{L^{1}} \\
& +\left\|\overline{\mathcal{S}}\left(z^{m, \delta}(\tau)-z^{m, \delta}\left(\tau_{\ell}\right)\right)\left[\overline{\mathcal{S}}\left(\Delta z_{\ell-1}^{m}\right)-\overline{\mathcal{S}}\left(\Delta z_{\ell-1}^{m, \delta}\right)\right] \overline{\mathcal{S}}\left(\Delta z_{\ell-2}^{m}\right) \cdots \overline{\mathcal{S}}\left(\Delta z_{k}^{m}\right) u^{m}\left(\tau_{k}\right)\right\|_{L^{1}} \\
& +\cdots+\left\|\overline{\mathcal{S}}\left(z^{m, \delta}(\tau)-z^{m, \delta}\left(\tau_{\ell}\right)\right) \cdots \overline{\mathcal{S}}\left(\Delta z_{k+1}^{m, \delta}\right)\left[\overline{\mathcal{S}}\left(\Delta z_{k}^{m}\right)-\overline{\mathcal{S}}\left(\Delta z_{k}^{m, \delta}\right)\right] u^{m}\left(\tau_{k}\right)\right\|_{L^{1}} \\
& \leq 2 \delta(\ell+1-k)\left\|f^{\prime}\right\|_{\infty}\left|u_{0}\right|_{\mathrm{BV}} .
\end{aligned}
$$

Taking the limit $\delta \downarrow 0$, shows that (4.7) holds in $L^{1}(\mathbb{R})$-sense.

4.2. The oscillating running max and min functions. Lemma 4.3 identifies a class of intervals over which an entropy solution with driving path $z^{m} \in I_{0}^{m}([0, T])$ experiences "oscillatory cancellations". In this section we construct an operator $\overline{\mathrm{orm}}_{m}: C_{0}([0, T]) \rightarrow I_{0}^{m}([0, T])$, cf. 4.15$)$, which yields a piecewise linear driving path $\overline{\mathrm{orm}}_{m}[z]$ that is free of Lemma 4.3 type (iii) "oscillatory cancellations", has smaller (or equal) total variation than $z^{m}$, and (under some assumptions) produces the same entropy solution at final time as $z^{m}$ does, cf. Theorem 4.5 . The further removal of type (i), (ii) "oscillatory cancellations" is postponed to Section 5.

Definition 4.3. For any $z \in C_{0}([0, T])$, we define the monotonically increasing functions $A^{+}[z], A^{-}[z]:[0, T] \rightarrow[0, T]$ by

$$
\begin{aligned}
& A^{+}[z](t):=\min \left\{s \in[0, t] \mid z(s)=M^{+}[z](t)\right\}, \\
& A^{-}[z](t):=\min \left\{s \in[0, t] \mid z(s)=M^{-}[z](t)\right\},
\end{aligned}
$$

and the monotonically increasing càdlàg functions $\bar{A}^{+}[z], \bar{A}^{-}[z]:[0, T] \rightarrow[0, T]$ by

$$
\begin{aligned}
& \bar{A}^{+}[z](t):= \begin{cases}\lim _{\delta \downarrow 0} A^{+}[z](t+\delta) & \text { if } t \in[0, T), \\
A^{+}[z](T) & \text { if } t=T,\end{cases} \\
& \bar{A}^{-}[z](t):=\left\{\begin{array}{lll}
\lim _{\delta \downarrow 0} A^{-}[z](t+\delta) & \text { if } t \in[0, T), \\
A^{-}[z](T) & \text { if } t=T .
\end{array}\right.
\end{aligned}
$$

For a given set of points

$$
0=\tau_{0}<\tau_{1}<\ldots<\tau_{m}=T,
$$

and $z^{m}=\mathcal{I}[z]\left(\cdot ;\left\{\tau_{k}\right\}_{k=0}^{m}\right)$, define

$$
A^{ \pm}\left[z^{m}\right]:=\bar{A}^{+}\left[z^{m}\right]\left(\left\{\tau_{k}\right\}_{k=0}^{m}\right) \cup \bar{A}^{-}\left[z^{m}\right]\left(\left\{\tau_{k}\right\}_{k=0}^{m}\right) \subset\left\{\tau_{k}\right\}_{j=0}^{m} .
$$

The set inclusion $A^{ \pm}\left[z^{m}\right] \subset\left\{\tau_{k}\right\}_{j=0}^{m}$ is verified in Lemma 4.4. 
Note that $0 \in A^{ \pm}\left[z^{m}\right]$, and let $\left\{\tau_{j(k)}\right\}_{k=0}^{\bar{m}}=A^{ \pm}\left[z^{m}\right] \cup\{T\}$, with $0=j(0)<\cdots<$ $j(\bar{m})=m$, represent the subsequence of interpolation points in ascending order. The operator $\overline{\mathrm{orm}}: C_{0}([0, T]) \rightarrow I_{0}^{m}([0, T])$ is defined by

$$
\begin{aligned}
& \overline{\operatorname{orm}}[z]\left(t ;\left\{\tau_{j(k)}\right\}_{k=0}^{\bar{m}}\right) \\
& =\sum_{k=0}^{\bar{m}-1} \mathbf{1}_{t \in\left(\tau_{j(k)}, \tau_{j(k+1)}\right]}\left(z\left(\tau_{j(k)}\right)+\left(z\left(\tau_{j(k+1)}\right)-z\left(\tau_{j(k)}\right)\right) \frac{t-\tau_{j(k)}}{\tau_{j(k+1)}-\tau_{j(k)}}\right) .
\end{aligned}
$$

We refer to $\overline{\mathrm{orm}}[z]\left(\cdot ;\left\{\tau_{j(k)}\right\}_{k=0}^{\bar{m}}\right)$ as the piecewise linear oscillating running max/min (orm) function of $z$, and frequently use the shorthand $\overline{\mathrm{orm}}_{m}[z]=\overline{\mathrm{orm}}[z]\left(\cdot ;\left\{\tau_{j(k)}\right\}_{k=0}^{\bar{m}}\right)$, when no confusion is possible.

See Figure 8 for an example illustrating $\bar{A}^{+}\left[z^{m}\right], \bar{A}^{-}\left[z^{m}\right]$, and $\overline{\mathrm{orm}}_{m}[z]$.
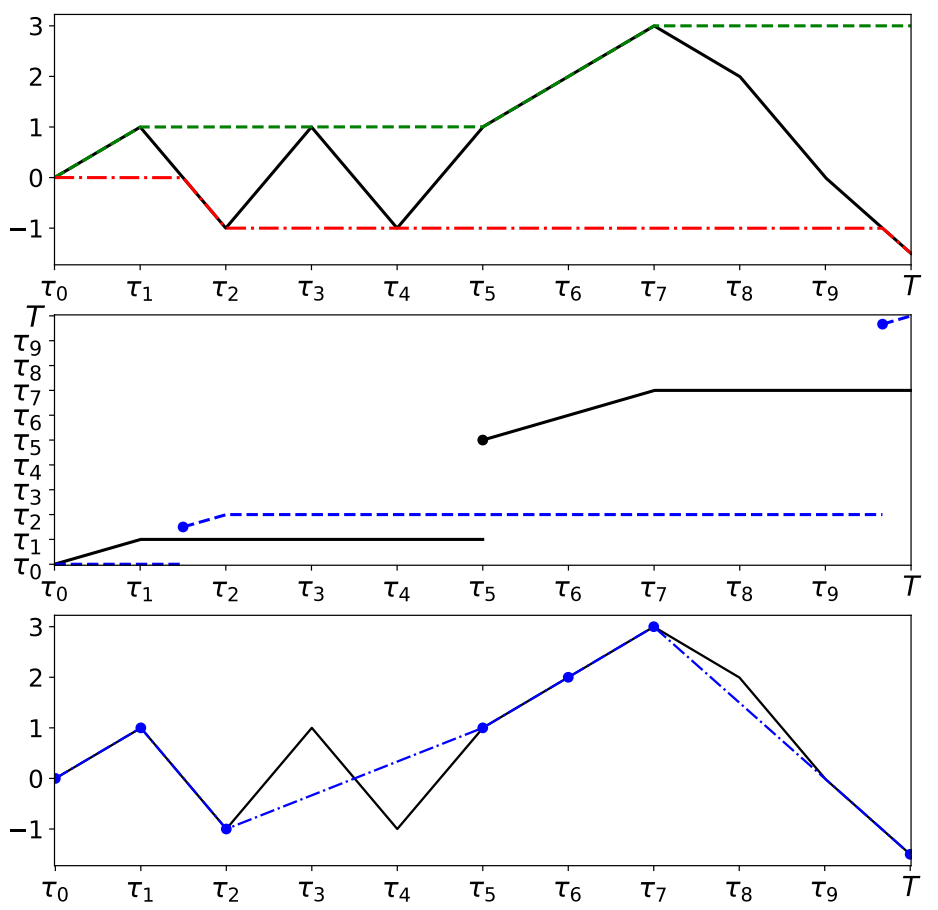

Figure 8. Top: The piecewise linear path $z^{m} \in I_{0}\left([0, T] ;\left\{\tau_{j}\right\}_{j=0}^{m}\right)$ with $m=10$ (black line) and the associated running maximum $M^{+}\left[z^{m}\right]$ (green dashed line) and running minimum $M^{-}\left[z^{m}\right]$ (red dash-dotted line). Middle: $\bar{A}^{+}\left[z^{m}\right]$ (black line) and $\bar{A}^{-}\left[z^{m}\right]$ (blue dashed line). Black and blue dots illustrate that the respective functions are right-continuous at jump-discontinuities. Bottom: The piecewise linear path $z^{m}$ (black line) and the associated $\overline{\mathrm{orm}}_{m}[z]$ (blue dash-dotted line). Blue dots mark the value of $\overline{\mathrm{orm}}_{m}[z]$ at its interpolation points $\left\{\tau_{j(k)}\right\}_{k=0}^{m}$.

For later reference we collect some properties of $\bar{A}^{ \pm}[z]$ in the following lemma:

Lemma 4.4. Assume that $z \in C_{0}([0, T])$. Then, for all $t \in[0, T]$,

$$
\max \left(\bar{A}^{+}[z], \bar{A}^{-}[z]\right)(t) \leq t
$$

$$
\begin{aligned}
& z\left(\bar{A}^{+}[z](t)\right)=M^{+}[z]\left(\bar{A}^{+}[z](t)\right)=M^{+}[z](t), \\
& z\left(\bar{A}^{-}[z](t)\right)=M^{-}[z]\left(\bar{A}^{-}[z](t)\right)=M^{-}[z](t),
\end{aligned}
$$




$$
\bar{A}^{+}[z]\left(\bar{A}^{+}[z](t)\right)=\bar{A}^{+}[z](t), \quad \bar{A}^{-}[z]\left(\bar{A}^{-}[z](t)\right)=\bar{A}^{-}[z](t) .
$$

Furthermore, for any set of points that satisfies

$$
0=\tau_{0}<\tau_{1}<\ldots<\tau_{m}=T, \quad m \geq 2,
$$

and $z^{m}=\mathcal{I}[z]\left(\cdot ;\left\{\tau_{k}\right\}_{k=0}^{m}\right)$, it holds that

$$
A^{ \pm}\left[z^{m}\right] \subset\left\{\tau_{k}\right\}_{k=0}^{m}
$$

and

$$
\tau=\max \left\{\bar{A}^{+}[z], \bar{A}^{-}[z]\right\}(\tau) \quad \text { for all } \tau \in A^{ \pm}\left[z^{m}\right] .
$$

Proof. By Definition 4.3 .

$$
\max \left(\bar{A}^{+}[z], \bar{A}^{-}[z]\right)(t)=\lim _{\delta \downarrow 0} \max \left(A^{+}[z], A^{-}[z]\right)(t+\delta) \leq \lim _{\delta \downarrow 0}(t+\delta)=t .
$$

To verify 4.16), $z \in C_{0}([0, T])$ implies that $M^{+}[z] \in C_{0}([0, T])$,

$$
\begin{aligned}
z\left(\bar{A}^{+}[z](t)\right) & =z\left(\lim _{\delta \downarrow 0} A^{+}[z](t+\delta)\right)=\lim _{\delta \downarrow 0} z\left(A^{+}[z](t+\delta)\right) \\
& =\lim _{\delta \downarrow 0} M^{+}[z](t+\delta)=M^{+}[z](t),
\end{aligned}
$$

and

$$
\begin{aligned}
M^{+}[z]\left(\bar{A}^{+}[z](t)\right) & =M^{+}[z]\left(\lim _{\delta \downarrow 0} A^{+}[z](t+\delta)\right)=\lim _{\delta \downarrow 0} M^{+}[z]\left(A^{+}[z](t+\delta)\right) \\
& =\lim _{\delta \downarrow 0} M^{+}[z](t+\delta)=M^{+}[z](t) .
\end{aligned}
$$

The second line of 4.16 follows by a similar argument.

To verify 4.17), we begin by noting that for all $t \in[0, T]$,

$$
\begin{aligned}
A^{+}[z]\left(A^{+}[z](t)\right) & =\min \left\{s \in\left[0, A^{+}[z](t)\right] \mid z(s)=M^{+}[z]\left(A^{+}[z](t)\right)\right\} \\
& =\min \left\{s \in\left[0, A^{+}[z](t)\right] \mid z(s)=M^{+}[z](t)\right\} \\
& =A^{+}[z](t) .
\end{aligned}
$$

By writing

$$
B_{1}^{+}[z]=\left\{t \in[0, T) \mid \lim _{\delta \downarrow 0} A^{+}[z](t+\delta)-A^{+}[z](t)=0\right\} \cup\{T\}
$$

and

$$
B_{2}^{+}[z]=\left\{t \in[0, T) \mid \lim _{\delta \downarrow 0} A^{+}[z](t+\delta)-A^{+}[z](t)>0\right\},
$$

we see that for all $t \in B_{1}^{+}[z], \bar{A}^{+}[z](t)=A^{+}[z](t)$. Hence,

$$
\bar{A}^{+}[z]\left(\bar{A}^{+}[z](t)\right) \leq \bar{A}^{+}[z](t)=A^{+}[z](t)
$$

and since $\bar{A}^{+}[z] \geq A^{+}[z]$,

$$
\bar{A}^{+}[z]\left(\bar{A}^{+}[z](t)\right) \geq A^{+}[z]\left(A^{+}[z](t)\right)=A^{+}[z](t) .
$$

We conclude that

$$
\bar{A}^{+}[z]\left(\bar{A}^{+}[z](t)\right)=A^{+}[z](t)=\bar{A}^{+}[z](t) \quad \forall t \in B_{1}^{+}[z] .
$$

We claim that for all $t \in B_{2}^{+}[z]$ and $\delta \in(0, T-t)$, it holds that $A^{+}[z](t+\delta)>t$; supposing otherwise, 4.20 and the monotonicity (increasing) of $A^{+}[z]$ leads to the following contradiction

$$
A^{+}[z](t+\delta)-A^{+}[z](t)=A^{+}[z]\left(A^{+}[z](t+\delta)\right)-A^{+}[z](t) \leq 0 .
$$

Hence, for all $t \in B_{2}^{+}[z]$, it follows by the preceding observation and $\bar{A}^{+}[z](t) \leq t$ that $\bar{A}^{+}[z]\left(\bar{A}^{+}[z](t)\right)=\bar{A}^{+}[z](t)$. The equality $\bar{A}^{-}[z]\left(\bar{A}^{-}[z](t)\right)=\bar{A}^{-}[z](t)$ can be verified by a similar argument.

To verify 4.18, observe that since $z^{m}$ is piecewise linear, it holds that

$$
A^{+}\left[z^{m}\right]\left(\left\{\tau_{k}\right\}_{k=0}^{m}\right) \cup A^{-}\left[z^{m}\right]\left(\left\{\tau_{k}\right\}_{k=0}^{m}\right) \subset\left\{\tau_{k}\right\}_{k=0}^{m},
$$


and for all $k \in\{0,1, \ldots, m\}$,

$$
\bar{A}^{+}\left[z^{m}\right]\left(\tau_{k}\right)= \begin{cases}A^{+}\left[z^{m}\right]\left(\tau_{k}\right) & \text { if } \tau_{k} \in B_{1}^{+}\left[z^{m}\right], \\ \tau_{k} & \text { if } \tau_{k} \in B_{2}^{+}\left[z^{m}\right],\end{cases}
$$

and

where

$$
\bar{A}^{-}\left[z^{m}\right]\left(\tau_{k}\right)= \begin{cases}A^{-}\left[z^{m}\right]\left(\tau_{k}\right) & \text { if } \tau_{k} \in B_{1}^{-}\left[z^{m}\right], \\ \tau_{k} & \text { if } \tau_{k} \in B_{2}^{-}\left[z^{m}\right]\end{cases}
$$

$$
B_{1}^{-}\left[z^{m}\right]=\left\{t \in[0, T) \mid \lim _{\delta \downarrow 0} A^{-}\left[z^{m}\right](t+\delta)-A^{-}\left[z^{m}\right](t)=0\right\} \cup\{T\}
$$

and

$$
B_{2}^{-}[z]=\left\{t \in[0, T) \mid \lim _{\delta \downarrow 0} A^{-}\left[z^{m}\right](t+\delta)-A^{-}\left[z^{m}\right](t)>0\right\} .
$$

To verify 4.19), equation 4.17) implies that

$$
\bar{A}^{ \pm}\left[z^{m}\right](\tau)=\tau \quad \forall \tau \in \bar{A}^{ \pm}\left[z^{m}\right]\left(\left\{\tau_{k}\right\}_{k=0}^{m}\right) .
$$

The next definition is an extension of the solution representation (4.6) from continuous to (jump-)discontinuous driving paths. This extension will come handy in the study of asymptotic properties of $\overline{\mathrm{orm}}_{m}$ when $m \rightarrow \infty$.

Definition 4.4. Let $\mathcal{D}([0, T])$ denote the space of càdlàg functions $g:[0, T] \rightarrow \mathbb{R}$. For some $m \geq 2$, let $\left\{\tau_{k}\right\}_{k=0}^{m}$ be a set of points with $0=\tau_{0}<\tau_{1}<\ldots<\tau_{m}=T$. Given $f \in C^{2}(\mathbb{R}), u_{0} \in\left(L^{1} \cap B V\right)(\mathbb{R})$ and $y \in \mathcal{D}([0, T])$, we define

$$
v\left(\tau_{k} ; y,\left\{\tau_{k}\right\}_{k=0}^{m}\right):= \begin{cases}u_{0} & \text { if } k=0 \\ \overline{\mathcal{S}}\left(\Delta y_{k-1}\right) \overline{\mathcal{S}}\left(\Delta y_{k-2}\right) \ldots \overline{\mathcal{S}}\left(\Delta y_{0}\right) u_{0} & \text { if } k \in\{1, \ldots, m\},\end{cases}
$$

where $\Delta y_{k}=y\left(\tau_{k+1}\right)-y\left(\tau_{k}\right)$.

Note that for any $f \in C^{2}(\mathbb{R}), u_{0} \in\left(L^{1} \cap B V\right)(\mathbb{R})$, set of points

$$
0=\tau_{0}<\tau_{1}<\ldots<\tau_{m}=T, \quad m \geq 2,
$$

and $y \in \mathcal{D}([0, T])$, it holds by $4.8,4.10$ and induction that

$$
\left\|v\left(\tau_{k} ; y,\left\{\tau_{r}\right\}_{r=0}^{m}\right)\right\|_{L^{1}} \leq\left\|u_{0}\right\|_{L^{1}} \quad\left|v\left(\tau_{k} ; y,\left\{\tau_{r}\right\}_{r=0}^{m}\right)\right|_{\mathrm{BV}} \leq\left|u_{0}\right|_{\mathrm{BV}} \quad \forall k \leq m .
$$

Moreover, cf. 4.6),

$$
v\left(T ; z,\left\{\tau_{k}\right\}_{k=0}^{m}\right)=v\left(T ; \mathcal{I}^{m}[z],\left\{\tau_{k}\right\}_{k=0}^{m}\right)=u^{m}(T), \quad z \in C_{0}([0, T]) .
$$

The next theorem shows that if the flux is strictly convex, then the driving paths $\mathcal{I}^{m}[z]$ and $\overline{\mathrm{orm}}_{m}[z]$ produce the same entropy solution at final time.

Theorem 4.5. Assume that $f \in C^{2}(\mathbb{R})$ is strictly convex, $u_{0} \in\left(L^{1} \cap B V\right)(\mathbb{R})$ and $z \in C_{0}([0, T])$. For some $m \geq 2$, let $\left\{\tau_{k}\right\}_{k=0}^{m}$ denote a set of points satisfying $0=\tau_{0}<\tau_{1}<\ldots<\tau_{m}=T, z^{m}=\mathcal{I}^{m}[z]$, and let

$$
0=\tau_{j(0)}<\tau_{j(1)}<\ldots<\tau_{j(\bar{m})}=T
$$

denote the associated interpolation points of $\overline{\mathrm{orm}}[z]\left(\cdot ;\left\{\tau_{j(k)}\right\}_{j=0}^{\bar{m}}\right)$, cf. Definition 4.3 . Then

$$
v\left(T ; \overline{\operatorname{orm}}_{m}[z],\left\{\tau_{j(k)}\right\}_{k=0}^{\bar{m}}\right)=v\left(T ; \overline{\operatorname{orm}}_{m}[z],\left\{\tau_{k}\right\}_{k=0}^{m}\right)=v\left(T ; z^{m},\left\{\tau_{k}\right\}_{k=0}^{m}\right) .
$$


Proof. Recall from the solution representations 4.6 and 4.21 that

$$
v\left(T ; z^{m},\left\{\tau_{k}\right\}_{k=0}^{m}\right)=u^{m}(T)=\overline{\mathcal{S}}\left(\Delta z_{m-1}^{m}\right) \cdots \overline{\mathcal{S}}\left(\Delta z_{0}^{m}\right) u_{0} .
$$

By writing $y^{m}=\overline{\mathrm{orm}}_{m}[z]$ and introducing the shorthand

$$
v^{m}\left(\tau_{k}\right):=v\left(\tau_{k} ; y^{m},\left\{\tau_{r}\right\}_{r=0}^{m}\right), \quad \forall k \in\{0,1, \ldots, m\},
$$

we have by 4.21,

$$
v^{m}(T)=\overline{\mathcal{S}}\left(\Delta y_{m-1}^{m}\right) \ldots \overline{\mathcal{S}}\left(\Delta y_{0}^{m}\right) u_{0} .
$$

For any $k \in\{0, \ldots, \bar{m}-1\},\left.y^{m}\right|_{\left[\tau_{j(k)}, \tau_{j(k+1)}\right]}$ is a linear function. Therefore, either

$$
\dot{y}^{m}(s+) \geq 0, \quad \forall s \in\left[\tau_{j(k)}, \tau_{j(k+1)}\right)
$$

or

$$
\dot{y}^{m}(s+)<0, \quad \forall s \in\left[\tau_{j(k)}, \tau_{j(k+1)}\right),
$$

and Lemma 4.3(i-ii) and $\left.y^{m}\right|_{A_{\tau}^{ \pm}\left[z^{m}\right] \cup\{T\}}=\left.z^{m}\right|_{A_{\tau}^{ \pm}\left[z^{m}\right] \cup\{T\}}$, cf. 4.15, yield

$$
\begin{aligned}
v^{m}(T) & =\overline{\mathcal{S}}\left(y^{m}\left(\tau_{j(\bar{m})}\right)-y^{m}\left(\tau_{j(\bar{m}-1)}\right)\right) \ldots \overline{\mathcal{S}}\left(y^{m}\left(\tau_{j(1)}\right)-y^{m}\left(\tau_{j(0)}\right)\right) u_{0} \\
& =\overline{\mathcal{S}}\left(z^{m}\left(\tau_{j(\bar{m})}\right)-z^{m}\left(\tau_{j(\bar{m}-1)}\right)\right) \ldots \overline{\mathcal{S}}\left(z^{m}\left(\tau_{j(1)}\right)-z^{m}\left(\tau_{j(0)}\right)\right) u_{0} .
\end{aligned}
$$

Assume $\bar{m}>1$ (otherwise $\left.z^{m}\right|_{\left[0, \tau_{m-1}\right]}=0$ and the lemma trivially holds). We claim that for all $k \in\{0, \ldots, \bar{m}-1\}$ such that $j(k+1)-j(k) \geq 2$,

$$
\begin{aligned}
\overline{\mathcal{S}}\left(z^{m}\left(\tau_{j(k+1)}\right)-z^{m}\left(\tau_{j(k)}\right)\right) u^{m}\left(\tau_{j(k)}\right) & =\overline{\mathcal{S}}\left(\Delta z_{j(k+1)-1}^{m}\right) \ldots \overline{\mathcal{S}}\left(\Delta z_{j(k)}^{m}\right) u^{m}\left(\tau_{j(k)}\right) \\
& =u^{m}\left(\tau_{j(k+1)}\right) .
\end{aligned}
$$

Define

$$
k_{1}=\min \{\{k \in\{0, \ldots, \bar{m}-1\} \mid j(k+1)-j(k) \geq 2\} \cup\{\bar{m}\}\} .
$$

Then, since $j(k+1)-j(k)=1$ for all $k<k_{1}$, it follows from the solution representations 4.6 and 4.21 that $v^{m}\left(\tau_{j\left(k_{1}\right)}\right)=u^{m}\left(\tau_{j\left(k_{1}\right)}\right)$. If $k_{1}=\bar{m}$, we have $v^{m}(T)=u^{m}(T)$. Otherwise, if $k_{1}<\bar{m}$, assumption 4.22 implies that $v^{m}\left(\tau_{j\left(k_{1}+1\right)}\right)=u^{m}\left(\tau_{j\left(k_{1}+1\right)}\right)$. Let

$$
k_{2}=\min \left\{\left\{k \in\left\{k_{1}+1, \ldots, \bar{m}-1\right\} \mid j(k+1)-j(k) \geq 2\right\} \cup\{\bar{m}\}\right\},
$$

and argue as above to conclude that if $k_{2}=\bar{m}$, then $v^{m}(T)=u^{m}(T)$, and otherwise, $v^{m}\left(\tau_{j\left(k_{2}+1\right)}\right)=u^{m}\left(\tau_{j\left(k_{2}+1\right)}\right)$. The lemma follows by induction once we have verified the claim 4.22.

Suppose $k \in\{0, \ldots, \bar{m}-1\}$ satisfies $j(k+1)-j(k) \geq 2$. Consider two cases: either $\tau_{j(k+1)} \notin A^{ \pm}\left[z^{m}\right]$ or $\tau_{j(k+1)} \in A^{ \pm}\left[z^{m}\right]$.

The case $\tau_{j(k+1)} \notin A^{ \pm}\left[z^{m}\right]$ is only possible if $k=\bar{m}-1$, i.e., if $\tau_{j(k+1)}=T$. Then

$z^{m}\left(\tau_{\ell}\right) \in\left[M^{-}\left[z^{m}\right]\left(\tau_{j(k)}\right), M^{+}\left[z^{m}\right]\left(\tau_{j(k)}\right)\right], \quad \forall \ell \in\{j(\bar{m}-1), j(\bar{m}-1)+1, \ldots, j(\bar{m})\}$, which implies that

$$
z^{m}\left(\left[\tau_{j(k)}, T\right]\right) \subset\left[M^{-}\left[z^{m}\right]\left(\tau_{j(k)}\right), M^{+}\left[z^{m}\right]\left(\tau_{j(k)}\right)\right],
$$

and 4.22 follows by Lemma 4.3 .

For the second case, $\tau_{j(k+1)} \in A^{ \pm}\left[z^{m}\right]$, then $z^{m}\left(\tau_{\ell}\right) \in\left[M^{-}\left[z^{m}\right]\left(\tau_{j(k)}\right), M^{+}\left[z^{m}\right]\left(\tau_{j(k)}\right)\right], \quad \forall \ell \in\{j(k), j(k)+1, \ldots, j(k+1)-1\}$, and there exists a unique $\tau \in\left(\tau_{j(k+1)-1}, \tau_{j(k+1)}\right]$ such that

$$
\begin{aligned}
& z^{m}\left(\left[\tau_{j(k)}, \tau\right]\right) \subset\left[M^{-}\left[z^{m}\right]\left(\tau_{j(k)}\right), M^{+}\left[z^{m}\right]\left(\tau_{j(k)}\right)\right], \\
& \tau \in\left\{\bar{A}^{+}\left[z^{m}\right](\tau), \bar{A}^{-}\left[z^{m}\right](\tau)\right\} .
\end{aligned}
$$


To show 4.23, if $z^{m}\left(\tau_{j(k+1)}\right) \in\left[M^{-}\left[z^{m}\right]\left(\tau_{j(k)}\right), M^{+}\left[z^{m}\right]\left(\tau_{j(k)}\right)\right]$ then $\tau=\tau_{j(k+1)}$. Otherwise, if $z^{m}\left(\tau_{j(k+1)}\right) \notin\left[M^{-}\left[z^{m}\right]\left(\tau_{j(k)}\right), M^{+}\left[z^{m}\right]\left(\tau_{j(k)}\right)\right]$, then

$z^{m}\left(\tau_{j(k+1)-1}\right) \in\left[M^{-}\left[z^{m}\right]\left(\tau_{j(k)}\right), M^{+}\left[z^{m}\right]\left(\tau_{j(k)}\right)\right]$ implies that $\left|\Delta z_{j(k+1)-1}^{m}\right|>0$. This observation and $\tau_{j(k+1)-1} \notin A^{ \pm}\left[z^{m}\right]$ verifies statement (4.23).

By 4.23 and Lemma 4.3

$$
\begin{aligned}
& \overline{\mathcal{S}}\left(\Delta z_{j(k+1)-1}^{m}\right) \ldots \overline{\mathcal{S}}\left(\Delta z_{j(k)}^{m}\right) u^{m}\left(\tau_{j(k)}\right) \\
& =\overline{\mathcal{S}}\left(\Delta z^{m}\left(\tau_{j(k+1)}\right)-z^{m}(\tau)\right) \overline{\mathcal{S}}\left(z^{m}(\tau)-z^{m}\left(\tau_{j(k+1)-1}\right)\right) \ldots \overline{\mathcal{S}}\left(\Delta z_{j(k)}^{m}\right) u^{m}\left(\tau_{j(k)}\right) \\
& =\overline{\mathcal{S}}\left(\Delta z^{m}\left(\tau_{j(k+1)}\right)-z^{m}(\tau)\right) \overline{\mathcal{S}}\left(z^{m}(\tau)-z^{m}\left(\tau_{j(k)}\right)\right) u^{m}\left(\tau_{j(k)}\right) .
\end{aligned}
$$

If $\tau_{j(k+1)}=\bar{A}^{+}\left[z^{m}\right]\left(\tau_{j(k+1)}\right)$, then it must hold that $\Delta z_{j(k+1)-1}^{m} \geq 0$, hence, $z^{m}\left(\tau_{j(k+1)}\right)-z^{m}(\tau) \geq 0$. The former inequality and $\tau \in\left\{\bar{A}^{+}\left[z^{m}\right](\tau), \bar{A}^{-}\left[z^{m}\right](\tau)\right\}$ imply that $z^{m}(\tau)=M^{+}\left[z^{m}\right](\tau)$, so that also $z^{m}(\tau)-z^{m}\left(\tau_{j(k)}\right) \geq 0$. If, on the other hand, $\tau_{j(k+1)}=\bar{A}^{-}\left[z^{m}\right]\left(\tau_{j(k+1)}\right)$, then a similar argument yields

$$
z^{m}\left(\tau_{j(k+1)}\right)-z^{m}(\tau) \leq 0 \quad \text { and } \quad z^{m}(\tau)-z^{m}\left(\tau_{j(k)}\right) \leq 0 .
$$

We conclude from the above that if $\tau_{j(k+1)} \in A^{ \pm}\left[z^{m}\right]$, then

$$
\left(z^{m}\left(\tau_{j(k+1)}\right)-z^{m}(\tau)\right)\left(z^{m}(\tau)-z^{m}\left(\tau_{j(k)}\right)\right) \geq 0 .
$$

Since both increments either are non-negative or non-positive,

$$
\overline{\mathcal{S}}\left(z^{m}\left(\tau_{j(k+1)}\right)-z^{m}(\tau)\right) \overline{\mathcal{S}}\left(z^{m}(\tau)-z^{m}\left(\tau_{j(k)}\right)\right) u^{m}\left(\tau_{j(k)}\right)
$$

equals the unique entropy solution at time

$$
t=\left|z^{m}\left(\tau_{j(k+1)}\right)-z^{m}(\tau)+\left(z^{m}(\tau)-z^{m}\left(\tau_{j(k)}\right)\right)\right|=\left|z^{m}\left(\tau_{j(k+1)}\right)-z^{m}\left(\tau_{j(k)}\right)\right|
$$

of

$$
\partial_{t} \tilde{u}+\operatorname{sign}\left(z^{m}\left(\tau_{j(k+1)}\right)-z^{m}\left(\tau_{j(k)}\right)\right) \partial_{x} f(\tilde{u})=0, \quad \tilde{u}(0)=u^{m}\left(\tau_{j(k)}\right),
$$

cf. 4.4. However,

$$
\overline{\mathcal{S}}\left(z^{m}\left(\tau_{j(k+1)}\right)-z^{m}\left(\tau_{j(k)}\right)\right) u^{m}\left(\tau_{j(k)}\right)
$$

also equals $\tilde{u}\left(\left|z^{m}\left(\tau_{j(k+1)}\right)-z^{m}\left(\tau_{j(k)}\right)\right|\right)$. Hence,

$$
\begin{aligned}
u^{m}\left(\tau_{j(k+1)}\right) & =\overline{\mathcal{S}}\left(\Delta z_{j(k+1)-1}^{m}\right) \cdots \overline{\mathcal{S}}\left(\Delta z_{j(k)}^{m}\right) u^{m}\left(\tau_{j(k)}\right) \\
& =\overline{\mathcal{S}}\left(\Delta z^{m}\left(\tau_{j(k+1)}\right)-z^{m}(\tau)\right) \overline{\mathcal{S}}\left(z^{m}(\tau)-z^{m}\left(\tau_{j(k)}\right)\right) u^{m}\left(\tau_{j(k)}\right) \\
& =\overline{\mathcal{S}}\left(z^{m}\left(\tau_{j(k+1)}\right)-z^{m}\left(\tau_{j(k)}\right)\right) u^{m}\left(\tau_{j(k)}\right) .
\end{aligned}
$$

4.3. The limit orm. The operator $\overline{\mathrm{orm}}_{m}: C_{0}([0, T]) \rightarrow I_{0}\left([0, T] ;\left\{\tau_{j}\right\}_{j=0}^{m}\right)$ introduced in Definition 4.3 maps every driving path $z \in C_{0}([0, T])$ to a less oscillatory driving path $y^{m}=\overline{\mathrm{orm}}_{m}[z]$. In Theorem 4.5 it is shown that, provided $u_{0} \in\left(L^{1} \cap B V\right)(\mathbb{R})$ and $f \in C^{2}(\mathbb{R})$ is strictly convex, the paths $z^{m}=\mathcal{I}^{m}[z]$ and $\overline{\mathrm{orm}}_{m}[z]$ are equivalent in the sense of preserving final time solutions:

$$
v\left(T ; \overline{\mathrm{orm}}_{m}[z],\left\{\tau_{k}\right\}_{k=0}^{m}\right)=v\left(T ; z^{m},\left\{\tau_{k}\right\}_{k=0}^{m}\right)=u^{m}(T),
$$

for all $z \in C_{0}([0, T])$ and meshes $\left\{\tau_{k}\right\}_{k=0}^{m}$ for $m \geq 2$.

We next introduce an operator orm $: C_{0}([0, T]): \rightarrow \mathcal{D}([0, T])$ that may be viewed as the "limit extension" of the $\overline{\mathrm{orm}}_{m}$ operators in the sense that

$$
v\left(T ; \operatorname{orm}\left[z^{m}\right],\left\{\tau_{k}\right\}_{k=0}^{m}\right)=v\left(T ; \overline{\operatorname{orm}}_{m}[z],\left\{\tau_{k}\right\}_{k=0}^{m}\right),
$$

cf. Theorem 4.7, and, under the more restrictive assumptions of Theorem 4.10

$$
\lim _{m \rightarrow \infty} v\left(T ; \operatorname{orm}[z],\left\{\tau_{k}\right\}_{k=0}^{m}\right)=u(T)
$$


Definition 4.5. We define the operator orm $[\cdot]: C_{0}([0, T]) \rightarrow \mathcal{D}([0, T])$ by $\operatorname{orm}[z](t):= \begin{cases}M^{+}[z](t) \mathbf{1}_{\bar{A}^{+}[z](t)>\bar{A}^{-}[z](t)}+M^{-}[z](t) \mathbf{1}_{\bar{A}^{-}[z](t)>\bar{A}^{+}[z](t)} & \text { if } t \in[0, T), \\ z(T) & \text { if } t=T,\end{cases}$ and refer to orm $[z]$ as the oscillating running $\max / \min$ (orm) function of $z$.

See Figure 9 for examples illustrating the orm $[z]$ functions.
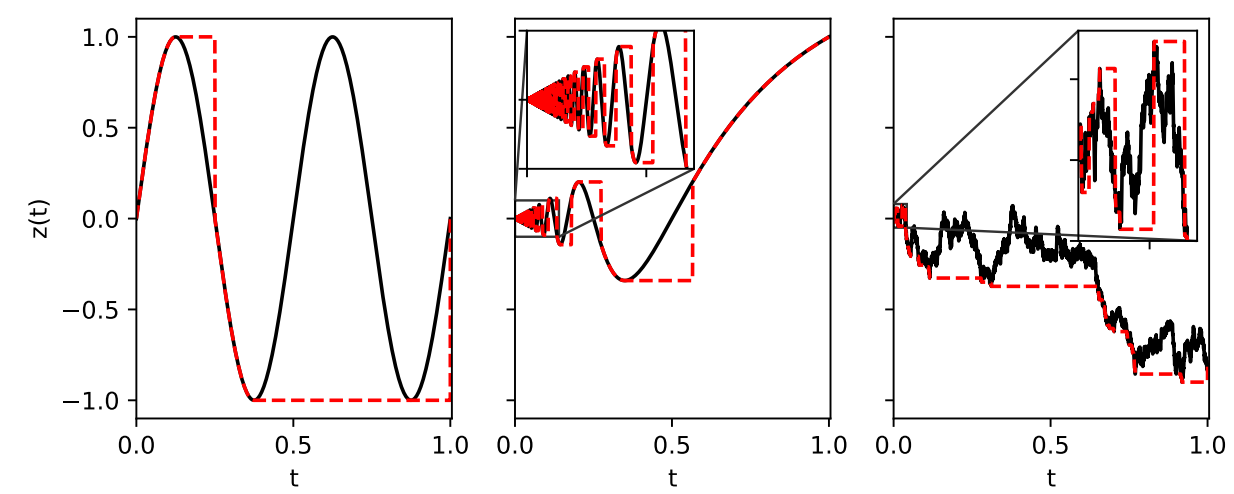

FiguRE 9. Illustration of a driving path $z$ (black line) and orm $[z]$ (red dashed line). Left plot: $z(t)=\sin (4 \pi t)$. Middle plot: $z(t)=\mathbf{1}_{t>0} t \sin (\pi /(2 t))$. Right plot: $z$ is a realization of a standard Wiener process.

It remains to verify that the orm functions are càdlàg.

Lemma 4.6. For all $z \in C_{0}([0, T])$, orm $[z] \in \mathcal{D}([0, T])$.

Proof. Note first that if $t \in \bar{A}^{+}[z]([0, T]) \cap \bar{A}^{-}[z]([0, T])$, then by 44.16),

$$
z(t)=M^{+}[z](t)=M^{-}[z](t) \Longrightarrow z(s)=0 \text { for all } s \in[0, t] .
$$

By 4.16 and Definition 4.5, we have for all $t \in[0, T)$,

$$
\begin{aligned}
\operatorname{orm}[z](t) & =M^{+}[z](t) \mathbf{1}_{\bar{A}^{+}[z](t)>\bar{A}^{-}[z](t)}+M^{-}[z](t) \mathbf{1}_{\bar{A}^{-}[z](t)>\bar{A}^{+}[z](t)} \\
& =\left\{\begin{array}{lll}
M^{+}[z](t)=z\left(\bar{A}^{+}[z](t)\right) & \text { if } & \bar{A}^{+}[z](t) \geq \bar{A}^{-}[z](t) \\
M^{-}[z](t)=z\left(\bar{A}^{-}[z](t)\right) & \text { if } & \bar{A}^{-}[z](t)>\bar{A}^{+}[z](t),
\end{array}\right.
\end{aligned}
$$

and $\operatorname{orm}[z](T)=z(T)$. Hence,

$$
\operatorname{orm}[z](t)= \begin{cases}z\left(\max \left(\bar{A}^{+}[z], \bar{A}^{-}[z]\right)(t)\right) & \text { if } t \in[0, T) \\ z(T) & \text { if } t=T\end{cases}
$$

and, since $z \in C_{0}([0, T])$ and

$$
g(t)=\mathbf{1}_{t<T} \max \left(\bar{A}^{+}[z], \bar{A}^{-}[z]\right)(t)+\mathbf{1}_{t=T} T
$$

is càdlàg whenever $\bar{A}^{+}[z], \bar{A}^{-}[z] \in \mathcal{D}([0, T])$, it follows that also orm $[z]=z \circ g$ belongs to $\mathcal{D}([0, T])$.

The next theorem shows that the mapping orm $[\cdot]$ is consistent in the sense that all the driving paths $z^{m}$, orm $\left[z^{m}\right]$, and $\overline{\operatorname{orm}}_{m}[z]$ produce the same entropy solution of (2.1) at the final time $T$. 
Theorem 4.7. Assume that $f \in C^{2}(\mathbb{R})$ is strictly convex, $u_{0} \in\left(L^{1} \cap B V\right)(\mathbb{R})$, and $z \in C_{0}([0, T])$. For some $m \geq 2$, let $\left\{\tau_{k}\right\}_{k=0}^{m}$ be a set of points satisfying

$$
0=\tau_{0}<\tau_{1}<\ldots<\tau_{m}=T
$$

$z^{m}=\mathcal{I}^{m}[z]$, and let

$$
0=\tau_{j(0)}<\tau_{j(1)}<\ldots<\tau_{j(\bar{m})}=T
$$

be the associated interpolation points of $\overline{\operatorname{orm}}[z]\left(\cdot ;\left\{\tau_{j(k)}\right\}_{k=0}^{\bar{m}}\right)$, cf. Definition 4.3 . Then

$$
v\left(T ; \operatorname{orm}\left[z^{m}\right],\left\{\tau_{j(k)}\right\}_{k=0}^{\bar{m}}\right)=v\left(T ; \operatorname{orm}\left[z^{m}\right],\left\{\tau_{k}\right\}_{k=0}^{m}\right)=u^{m}(T) .
$$

Proof. Set $y^{m}=\overline{\operatorname{orm}}_{m}[z]$ and $\hat{y}^{m}=\operatorname{orm}\left[z^{m}\right]$. By 4.19, , 4.24) and 4.15, it holds for all $k \in\{0,1, \ldots, \bar{m}-1\}$ that

$$
\hat{y}^{m}\left(\tau_{j(k)}\right)=z^{m}\left(\max \left(\bar{A}^{+}\left[z^{m}\right], \bar{A}^{-}\left[z^{m}\right]\right)\left(\tau_{j(k)}\right)\right)=z^{m}\left(\tau_{j(k)}\right)=y^{m}\left(\tau_{j(k)}\right),
$$

and

By Theorem 4.5

$$
\hat{y}^{m}\left(\tau_{j(\bar{m})}\right)=z^{m}(T)=y^{m}\left(\tau_{j(\bar{m})}\right) .
$$

$$
\begin{aligned}
& \overline{\mathcal{S}}\left(\Delta y_{\tau_{j(\bar{m})-1}^{m}}^{m} \cdots \overline{\mathcal{S}}\left(\Delta y_{\tau_{j(0)}^{m}}^{m}\right) u_{0}\right. \\
& \quad=\overline{\mathcal{S}}\left(y^{m}\left(\tau_{j(\bar{m})}\right)-y^{m}\left(\tau_{j(\bar{m}-1)}\right)\right) \cdots \overline{\mathcal{S}}\left(y^{m}\left(\tau_{j(1)}\right)-y^{m}\left(\tau_{j(0)}\right)\right) u_{0}=u^{m}(T)
\end{aligned}
$$

so to verify 4.25), it suffices to show that for all $k \in\{0,1, \ldots, \bar{m}-1\}$,

$$
\begin{aligned}
\overline{\mathcal{S}}\left(\Delta \hat{y}_{j(k+1)-1}^{m}\right) \ldots \overline{\mathcal{S}}\left(\Delta \hat{y}_{j(k)}^{m}\right) u^{m}\left(\tau_{j(k)}\right) & \\
\quad & \overline{\mathcal{S}}\left(y^{m}\left(\tau_{j(k+1)}\right)-y^{m}\left(\tau_{j(k)}\right)\right) u^{m}\left(\tau_{j(k)}\right) .
\end{aligned}
$$

For $k \in\{0,1, \ldots, \bar{m}-1\}$ such that $j(k+1)-j(k)=1$, 4.28) holds by 4.26).

Assume next that $k \in\{0,1, \ldots, \bar{m}-1\}$ is such that $j(k+1)-j(k) \geq 2$. If $k=\bar{m}-1$ and $\tau_{j(k+1)}=T \notin A^{ \pm}\left[z^{m}\right]$, then

$$
z^{m}\left(\left[\tau_{j(k)}, T\right]\right) \subset\left[M^{-}\left[z^{m}\right]\left(\tau_{j(k)}\right), M^{+}\left[z^{m}\right]\left(\tau_{j(k)}\right)\right] .
$$

It follows that $M^{+}\left[z^{m}\right], M^{-}\left[z^{m}\right], \bar{A}^{+}\left[z^{m}\right]$ and $\bar{A}^{-}\left[z^{m}\right]$ are constant over $\left[\tau_{j(\bar{m}-1)}, T\right)$, which implies that $\hat{y}^{m}$ is constant over $\left[\tau_{j(k)}, T\right)$. Hence $\hat{y}^{m}\left(\tau_{j(\bar{m})-1}\right)=\hat{y}^{m}\left(\tau_{j(\bar{m}-1)}\right)$,

$$
\Delta \hat{y}_{j(\bar{m})-\ell}^{m}=0 \quad \forall \ell \in\{2, \ldots, j(\bar{m})-j(\bar{m}-1)\},
$$

and since $\overline{\mathcal{S}}(0)=I$,

$$
\begin{aligned}
\overline{\mathcal{S}}\left(\Delta \hat{y}_{j(\bar{m})-1}^{m}\right) \cdots \overline{\mathcal{S}}\left(\Delta \hat{y}_{j(\bar{m}-1)}^{m}\right) u^{m}\left(\tau_{j(\bar{m}-1)}\right) \\
\quad=\overline{\mathcal{S}}\left(\Delta \hat{y}_{j(\bar{m})-1}^{m}\right) u^{m}\left(\tau_{j(\bar{m}-1)}\right) \\
\quad=\overline{\mathcal{S}}\left(y^{m}\left(\tau_{j(\bar{m})}\right)-y^{m}\left(\tau_{j(\bar{m}-1)}\right)\right) u^{m}\left(\tau_{j(\bar{m}-1)}\right) .
\end{aligned}
$$

Otherwise, if $k \in\{0,1, \ldots, \bar{m}-1\}$ is such that $j(k+1)-j(k) \geq 2$ and $\tau_{j(k+1)} \in$ $A^{ \pm}[z]$, then we recall from the proof of Theorem 4.5 that there exists a unique $\tau \in\left(\tau_{j(k+1)-1}, \tau_{j(k+1)}\right]$ such that

$$
z^{m}\left(\left[\tau_{j(k)}, \tau\right]\right) \subset\left[M^{-}\left[z^{m}\right]\left(\tau_{j(k)}\right), M^{+}\left[z^{m}\right]\left(\tau_{j(k)}\right)\right], \quad \tau \in\left\{\bar{A}^{+}(\tau), \bar{A}^{-}(\tau)\right\} .
$$

This implies that $\hat{y}^{m}(\tau)=z^{m}(\tau)$, cf. (4.24), and that $M^{+}\left[z^{m}\right], M^{-}\left[z^{m}\right], \bar{A}^{+}\left[z^{m}\right]$, $\bar{A}^{-}\left[z^{m}\right]$ are constant over the interval $\left[\tau_{j(k)}, \tau\right)$. Consequently, $\hat{y}^{m}$ is constant over $\left[\tau_{j(k)}, \tau\right)$, and since $\tau_{j(k+1)-1}<\tau, \hat{y}^{m}\left(\tau_{j(k+1)-1}\right)=\hat{y}^{m}\left(\tau_{j(k)}\right)$ and

$$
\Delta \hat{y}_{j(k+1)-\ell}^{m}=0 \quad \forall \ell \in\{2, \ldots, j(k+1)-j(k)\} .
$$

Hence,

$$
\begin{aligned}
\overline{\mathcal{S}}\left(\Delta \hat{y}_{j(k+1)-1}^{m}\right) \cdots \overline{\mathcal{S}}\left(\Delta \hat{y}_{j(k)}^{m}\right) u^{m}\left(\tau_{j(k)}\right) & =\overline{\mathcal{S}}\left(\Delta \hat{y}_{j(k+1)-1}^{m}\right) u^{m}\left(\tau_{j(k)}\right) \\
& =\overline{\mathcal{S}}\left(y^{m}\left(\tau_{j(k+1)}\right)-y^{m}\left(\tau_{j(k)}\right)\right) u^{m}\left(\tau_{j(k)}\right)
\end{aligned}
$$


An immediate consequence of the preceding proof is that the total variation of $\operatorname{orm}\left[\mathcal{I}^{m}[z]\right]$ is equal to the total variation of $\overline{\operatorname{orm}}_{m}[z]$.

Lemma 4.8. For any $z \in C_{0}([0, T])$ and points $0=\tau_{0}<\tau_{1}<\ldots<\tau_{m}=T$, $m \geq 2$, it holds that

$$
\left|\operatorname{orm}\left[\mathcal{I}[z]\left(\cdot ;\left\{\tau_{k}\right\}_{k=0}^{m}\right)\right]\right|_{\mathrm{BV}([0, T])}=\left|\overline{\operatorname{orm}}[z]\left(\cdot ;\left\{\tau_{k}\right\}_{k=0}^{m}\right)\right|_{\mathrm{BV}([0, T])} .
$$

Proof. Recall that $y^{m}=\overline{\mathrm{orm}}_{m}[z] \in I_{0}\left([0, T] ;\left\{\tau_{k}\right\}_{k=0}^{m}\right)$ is piecewise linear, with interpolation points $0=\tau_{j(0)}<\tau_{j(1)}<\ldots<\tau_{j(\bar{m})}=T$. Hence, $y^{m}$ is monotone over each interval $\left[\tau_{j(k)}, \tau_{j(k+1)}\right]$. Since $z^{m}=\mathcal{I}^{m}[z]$ is linear over every interval $\left[\tau_{k}, \tau_{k+1}\right]$, it follows by Definition 4.5 that $\hat{y}^{m}=\operatorname{orm}\left[z^{m}\right]$ is monotone over every interval $\left[\tau_{k}, \tau_{k+1}\right]$, and by the proof of Theorem 4.7 for every interval $\left[\tau_{j(k)}, \tau_{j(k+1)}\right]$ with $j(k+1)-j(k) \geq 2$, it holds that $\hat{y}^{m}$ is constant over $\left[\tau_{j(k)}, \tau_{j(k+1)-1}\right]$. Consequently, $\hat{y}^{m}$ is monotone over each interval $\left[\tau_{j(k)}, \tau_{j(k+1)}\right]$, whereby

$$
\left|\hat{y}^{m}\right|_{\mathrm{BV}\left(\left[\tau_{j(k)}, \tau_{j(k+1)}\right]\right)}=\left|\hat{y}^{m}\left(\tau_{j(k+1)}\right)-\hat{y}^{m}\left(\tau_{j(k)}\right)\right| \quad \forall k \in\{0,1, \ldots, \bar{m}-1\} .
$$

By 4.26,

$$
\begin{aligned}
\left|\hat{y}^{m}\right|_{\mathrm{BV}([0, T])} & =\sum_{k=0}^{\bar{m}-1}\left|\hat{y}^{m}\right|_{\mathrm{BV}\left(\left[\tau_{j(k)}, \tau_{j(k+1)}\right]\right)} \\
& =\sum_{k=0}^{\bar{m}-1}\left|\hat{y}^{m}\left(\tau_{j(k+1)}\right)-\hat{y}^{m}\left(\tau_{j(k)}\right)\right| \\
& =\sum_{k=0}^{\bar{m}-1}\left|y^{m}\left(\tau_{j(k+1)}\right)-y^{m}\left(\tau_{j(k)}\right)\right|=\left|y^{m}\right|_{\mathrm{BV}([0, T])} .
\end{aligned}
$$

Definition 4.6. For any mesh $\left\{\tau_{k}\right\}_{k=0}^{m}$ such that

$$
0=\tau_{0}<\tau_{1}<\cdots<\tau_{m}=T,
$$

and $g \in \mathcal{D}([0, T])$ we define the total variation of $g$ restricted to $\left\{\tau_{k}\right\}_{k=0}^{m}$ by

$$
|g|_{\mathrm{BV}\left(\left\{\tau_{k}\right\}_{k=0}^{m}\right)}:=\sum_{k=0}^{m-1}\left|g\left(\tau_{k+1}\right)-g\left(\tau_{k}\right)\right| .
$$

In settings with multi-layered notation, we will for the sake of readability employ the alternative notation

$$
\operatorname{TV}\left(g ;\left\{\tau_{k}\right\}_{k=0}^{m}\right)=|g|_{\mathrm{BV}\left(\left\{\tau_{k}\right\}_{k=0}^{m}\right)} .
$$

The next lemma shows that the approximation $v\left(T ; \operatorname{orm}[z],\left\{\tau_{j}\right\}_{j=0}^{m}\right)$ converges to a limit in $L^{1}(\mathbb{R})$ as $m \rightarrow \infty$, and it provides an upper bound for the approximation error. The upper bound depends on orm $[z]$, the mesh $\left\{\tau_{k}\right\}_{k=0}^{m},\left|u_{0}\right|_{\mathrm{BV}((\mathbb{R}))}$ and $\left\|f^{\prime}\right\|_{\infty}$, hence it differs from the stability result (2.3).

Lemma 4.9. Let $\left\{\left\{\tau_{k}^{m}\right\}_{k=0}^{\ell_{m}}\right\}_{m=0}^{\infty}$ denote a sequence of nested meshes fulfilling $\ell_{0} \geq$ 2 , and for all $m \geq 0$,

$$
0=\tau_{0}^{m}<\tau_{1}^{m}<\cdots<\tau_{\ell_{m}}^{m}=T, \text { with } \ell_{m}<\ell_{m+1} \leq 2 \ell_{m}
$$

and the nested mesh property

$$
\left\{\tau_{k}^{m}\right\}_{k=0}^{\ell_{m}} \subset\left\{\tau_{k}^{m+1}\right\}_{k=0}^{\ell_{m+1}}
$$


with a strictly increasing mapping $j^{m}:\left\{0,1, \ldots, \ell_{m}\right\} \rightarrow\left\{0,1, \ldots, \ell_{m+1}\right\}$ such that

$$
\tau_{j^{m}(k)}^{m+1}=\tau_{k}^{m}, \quad \forall k \in\left\{0,1, \ldots, \ell_{m}\right\},
$$

and

$$
1 \leq j^{m}(k+1)-j^{m}(k) \leq 2, \quad \forall k \in\left\{0,1, \ldots, \ell_{m}-1\right\} .
$$

Assume $f \in C^{2}(\mathbb{R})$ is strictly convex, $u_{0} \in\left(L^{1} \cap B V\right)(\mathbb{R}), z \in C_{0}([0, T])$. Then, for any $\widehat{m}, \bar{m} \in \mathbb{N} \cup\{0\}$ with $\widehat{m} \geq \bar{m}$,

$$
\begin{aligned}
& \left\|v\left(T ; \operatorname{orm}[z],\left\{\tau_{k}^{\widehat{m}}\right\}_{k=0}^{\ell_{\widehat{m}}}\right)-v\left(T ; \operatorname{orm}[z],\left\{\tau_{k}^{\bar{m}}\right\}_{k=0}^{\ell_{\bar{m}}}\right)\right\|_{L^{1}(\mathbb{R})} \\
& \quad \leq 2\left\|f^{\prime}\right\|_{\infty}\left|u_{0}\right|_{\mathrm{BV}(\mathbb{R})}\left(\operatorname{TV}\left(\operatorname{orm}[z] ;\left\{\tau_{k}^{\widehat{m}}\right\}_{k=0}^{\ell_{\widehat{m}}}\right)-\mathrm{TV}\left(\operatorname{orm}[z] ;\left\{\tau_{k}^{\bar{m}}\right\}_{k=0}^{\ell_{\bar{m}}}\right)\right) .
\end{aligned}
$$

Moreover, if

$$
|\operatorname{orm}[z]|_{\mathrm{BV}([0, T])}<\infty
$$

and

$$
\lim _{m \rightarrow \infty}\left(|\operatorname{orm}[z]|_{\mathrm{BV}([0, T])}-\mathrm{TV}\left(\operatorname{orm}[z] ;\left\{\tau_{k}^{m}\right\}_{k=0}^{\ell_{m}}\right)\right)=0,
$$

then

$$
v_{\text {orm }}(T):=\lim _{m \rightarrow \infty} v\left(T ; \operatorname{orm}[z],\left\{\tau_{k}^{m}\right\}_{k=0}^{\ell_{m}}\right) \in L^{1}(\mathbb{R})
$$

and

$$
\begin{aligned}
& \left\|v_{\text {orm }}(T)-v\left(T ; \operatorname{orm}[z],\left\{\tau_{k}^{m}\right\}_{k=0}^{\ell_{m}}\right)\right\|_{1} \\
& \quad \leq 2\left\|f^{\prime}\right\|_{\infty}\left|u_{0}\right|_{\mathrm{BV}(\mathbb{R})}\left(|\operatorname{orm}[z]|_{\mathrm{BV}([0, T])}-\mathrm{TV}\left(\operatorname{orm}[z] ;\left\{\tau_{k}^{m}\right\}_{k=0}^{\ell_{m}}\right)\right) .
\end{aligned}
$$

Proof. For a fixed $m \geq 0$, set $y=\operatorname{orm}[z], \Delta y_{k}^{m}=y\left(\tau_{k+1}^{m}\right)-y\left(\tau_{k}^{m}\right)$, and introduce the shorthand

$$
v^{m}\left(\tau_{k}^{m}\right)=v\left(\tau_{k}^{m} ; y,\left\{\tau_{j}^{m}\right\}_{j=0}^{\ell_{m}}\right), \quad \text { for all } k \in\left\{0,1, \ldots, \ell_{m}\right\} .
$$

We have that

$$
\begin{aligned}
& \left\|v^{m+1}(T)-v^{m}(T)\right\|_{L^{1}(\mathbb{R})} \\
& \quad=\left\|\overline{\mathcal{S}}\left(\Delta y_{\ell_{m+1}-1}^{m+1}\right) \cdots \overline{\mathcal{S}}\left(\Delta y_{0}^{m+1}\right) u_{0}-\overline{\mathcal{S}}\left(\Delta y_{\ell_{m}-1}^{m}\right) \cdots \overline{\mathcal{S}}\left(\Delta y_{0}^{m}\right) u_{0}\right\|_{L^{1}(\mathbb{R})} \\
& \quad \leq \sum_{k \in J_{2}^{m}}\left\|\Delta \overline{\mathcal{S}}_{k} u_{0}\right\|_{L^{1}(\mathbb{R})},
\end{aligned}
$$

where $J_{2}^{m}=\left\{k \in\left\{0,1, \ldots, \ell_{m}-1\right\} \mid j^{m}(k+1)-j^{m}(k)=2\right\}$ and

$\Delta \overline{\mathcal{S}}_{k} u_{0}:=$

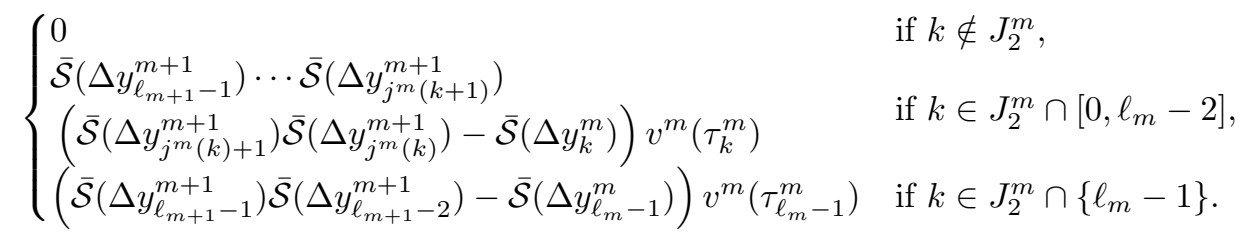

Assume that $k \in J_{2}^{m}$. Using (4.8), we obtain the following bound

$$
\left\|\Delta \overline{\mathcal{S}}_{k} u_{0}\right\|_{L^{1}(\mathbb{R})} \leq\left\|\left(\overline{\mathcal{S}}\left(\Delta y_{j^{m}(k)+1}^{m+1}\right) \overline{\mathcal{S}}\left(\Delta y_{j^{m}(k)}^{m+1}\right)-\overline{\mathcal{S}}\left(\Delta y_{k}^{m}\right)\right) v^{m}\left(\tau_{k}^{m}\right)\right\|_{L^{1}(\mathbb{R})} .
$$

We recall from 4.4 that $\overline{\mathcal{S}}\left(\Delta y_{j^{m}(k)+1}^{m+1}\right) \overline{\mathcal{S}}\left(\Delta y_{j^{m}(k)}^{m+1}\right) v^{m}\left(\tau_{k}^{m}\right)$ is recursively defined by being the solution at time $t=\left|\Delta y_{j^{m}(k)+1}^{m+1}\right|$ of

$$
\partial_{t} \tilde{u}+\operatorname{sign}\left(\Delta y_{j^{m}(k)+1}^{m+1}\right) \partial_{x} f(\tilde{u})=0, \quad t>0, \quad \tilde{u}(0)=\overline{\mathcal{S}}\left(\Delta y_{j^{m}(k)}^{m+1}\right) v^{m}\left(\tau_{k}^{m}\right) .
$$


If $\Delta y_{j^{m}(k)+1}^{m+1} \Delta y_{j^{m}(k)}^{m+1} \geq 0$, then

$$
\begin{aligned}
\overline{\mathcal{S}}\left(\Delta y_{j^{m}(k)+1}^{m+1}\right) \overline{\mathcal{S}}\left(\Delta y_{j^{m}(k)}^{m+1}\right) v^{m}\left(\tau_{k}^{m}\right) & =\overline{\mathcal{S}}\left(\Delta y_{j^{m}}^{m+1}(k)+1\right. \\
& =\overline{\mathcal{S}}\left(\Delta y_{k}^{m}\right) v^{m}\left(\tau_{k}^{m}\right) .
\end{aligned}
$$

This yields

$$
\left\|\Delta \overline{\mathcal{S}}_{k} u_{0}\right\|_{L^{1}(\mathbb{R})}=0, \quad \forall k \in\left\{\bar{k} \in J_{2}^{m} \mid \Delta y_{j^{m}(\bar{k})+1}^{m+1} \Delta y_{j^{m}(\bar{k})}^{m+1} \geq 0\right\}
$$

Consider next the case $\Delta y_{j^{m}(k)+1}^{m+1} \Delta y_{j^{m}(k)}^{m+1}<0$. In view of Definition $4.5, y\left(\tau_{k+1}^{m}\right) \in$ $\left\{M^{+}[z]\left(\tau_{k+1}^{m}\right), M^{-}[z]\left(\tau_{k+1}^{m}\right)\right\}$. If $y\left(\tau_{k+1}^{m}\right)=M^{+}[z]\left(\tau_{k+1}^{m}\right)$, then

$$
y\left(\tau_{\left.j^{m}(k)+1\right)}^{m+1}\right) \leq y\left(\tau_{k+1}^{m}\right)=y\left(\tau_{\left.j^{m}(k+1)\right)}^{m+1}\right),
$$

while if $y\left(\tau_{k+1}^{m}\right)=M^{-}[z]\left(\tau_{k+1}^{m}\right)$, then

$$
y\left(\tau_{j^{m}(k)+1}^{m+1}\right) \geq y\left(\tau_{k+1}^{m}\right)=y\left(\tau_{\left.j^{m}(k+1)\right)}^{m+1}\right) .
$$

We conclude that regardless of whether $y\left(\tau_{k+1}^{m}\right)=M^{+}[z]\left(\tau_{k+1}^{m}\right)$ or $y\left(\tau_{k+1}^{m}\right)=$ $M^{-}[z]\left(\tau_{k+1}^{m}\right)$, it holds that

$$
\Delta y_{j^{m}(k)+1}^{m+1} \Delta y_{k}^{m}=\left(y\left(\tau_{k+1}^{m}\right)-y\left(\tau_{j^{m}(k)+1}^{m+1}\right)\right)\left(y\left(\tau_{k+1}^{m}\right)-y\left(\tau_{k}^{m}\right)\right) \geq 0 .
$$

And since

$$
\operatorname{sign}\left(\Delta y_{k}^{m}\left(-\Delta y_{j^{m}(k)}^{m+1}\right)\right)=\operatorname{sign}\left(\Delta y_{k}^{m} \Delta y_{j^{m}(k)+1}^{m+1}\right) \geq 0
$$

(in the currently considered case $\left.\Delta y_{j^{m}(k)+1}^{m+1} \Delta y_{j^{m}(k)}^{m+1}<0\right)$, we also have that

$$
\Delta y_{k}^{m}\left(-\Delta y_{j^{m}(k)}^{m+1}\right) \geq 0 .
$$

Using the relationship

$$
\Delta y_{j^{m}(k)+1}^{m+1}=\Delta y_{k}^{m}-\Delta y_{j^{m}(k)}^{m+1}
$$

we obtain

$$
\begin{aligned}
\overline{\mathcal{S}}\left(\Delta y_{k}^{m}\right) \overline{\mathcal{S}} & \left(-\Delta y_{j^{m}(k)}^{m+1}\right) \overline{\mathcal{S}}\left(\Delta y_{j^{m}(k)}^{m+1}\right) v^{m}\left(\tau_{k}^{m}\right) \\
& =\overline{\mathcal{S}}\left(\Delta y_{k}^{m}-\Delta y_{j^{m}(k)}^{m+1}\right) \overline{\mathcal{S}}\left(\Delta y_{j^{m}(k)}^{m+1}\right) v^{m}\left(\tau_{k}^{m}\right) \\
& =\overline{\mathcal{S}}\left(\Delta y_{j^{m}(k)+1}^{m+1}\right) \overline{\mathcal{S}}\left(\Delta y_{j^{m}(k)}^{m+1}\right) v^{m}\left(\tau_{k}^{m}\right) .
\end{aligned}
$$

By 4.8, 4.9 and 4.10, we derive the following bound for all $k \in J_{2}^{m}$ such that $\Delta y_{j^{m}(k)+1}^{m+1} \Delta y_{j^{m}(k)}^{m+1}<0$,

$$
\begin{aligned}
\left\|\Delta \overline{\mathcal{S}}_{k} u_{0}\right\|_{L^{1}(\mathbb{R})} \leq & \left\|\overline{\mathcal{S}}\left(\Delta y_{k}^{m}\right)\left(\overline{\mathcal{S}}\left(-\Delta y_{j^{m}(k)}^{m+1}\right) \overline{\mathcal{S}}\left(\Delta y_{j^{m}(k)}^{m+1}\right)-I\right) v^{m}\left(\tau_{k}^{m}\right)\right\|_{L^{1}(\mathbb{R})} \\
\leq & \left\|\left(\overline{\mathcal{S}}\left(-\Delta y_{j^{m}(k)}^{m+1}\right) \overline{\mathcal{S}}\left(\Delta y_{j^{m}(k)}^{m+1}\right)-I\right) v^{m}\left(\tau_{k}^{m}\right)\right\|_{L^{1}(\mathbb{R})} \\
\leq & \left\|\left(\overline{\mathcal{S}}\left(-\Delta y_{j^{m}(k)}^{m+1}\right)-I\right) \overline{\mathcal{S}}\left(\Delta y_{j^{m}(k)}^{m+1}\right) v^{m}\left(\tau_{k}^{m}\right)\right\|_{L^{1}(\mathbb{R})} \\
& \quad+\left\|\left(\overline{\mathcal{S}}\left(\Delta y_{j^{m}(k)}^{m+1}\right)-I\right) v^{m}\left(\tau_{k}^{m}\right)\right\|_{L^{1}(\mathbb{R})} \\
\leq & 2\left\|f^{\prime}\right\|_{\infty}\left|u_{0}\right|_{\mathrm{BV}(\mathbb{R})}\left|\Delta y_{j^{m}(k)}^{m+1}\right| .
\end{aligned}
$$


By 4.34, 4.36) and 4.38,

$$
\begin{aligned}
\left\|v^{m+1}(T)-v^{m}(T)\right\|_{L^{1}(\mathbb{R})} & \leq \sum_{k \in J_{2}^{m}} 2\left\|f^{\prime}\right\|_{\infty}\left|u_{0}\right|_{\mathrm{BV}(\mathbb{R})}\left|\Delta y_{j^{m}(k)}^{m+1}\right| \\
& \leq 2\left\|f^{\prime}\right\|_{\infty}\left|u_{0}\right|_{\mathrm{BV}(\mathbb{R})}\left(\sum_{k=0}^{\ell_{m+1}}\left|\Delta y_{k}^{m+1}\right|-\sum_{k=0}^{\ell_{m}}\left|\Delta y_{k}^{m}\right|\right) .
\end{aligned}
$$

For $m_{2}>m_{1}$ we get

$$
\begin{aligned}
\left\|v^{m_{2}}(T)-v^{m_{1}}(T)\right\|_{L^{1}(\mathbb{R})} & \leq \sum_{m=m_{1}}^{m_{2}-1}\left\|v^{m+1}(T)-v^{m}(T)\right\|_{L^{1}(\mathbb{R})} \\
& \leq 2\left\|f^{\prime}\right\|_{\infty}\left|u_{0}\right|_{\mathrm{BV}(\mathbb{R})} \sum_{m=m_{1}}^{m_{2}-1}\left(\sum_{k=0}^{\ell_{m+1}}\left|\Delta y_{k}^{m+1}\right|-\sum_{k=0}^{\ell_{m}}\left|\Delta y_{k}^{m}\right|\right) \\
& =2\left\|f^{\prime}\right\|_{\infty}\left|u_{0}\right|_{\mathrm{BV}(\mathbb{R})}\left(\sum_{k=0}^{\ell_{m_{2}+1}}\left|\Delta y_{k}^{m_{2}+1}\right|-\sum_{k=0}^{\ell_{m_{1}}}\left|\Delta y_{k}^{m_{1}}\right|\right)
\end{aligned}
$$

By assumption 4.32, both sums on the right converge to $\mid$ orm $\left.[z]\right|_{\mathrm{BV}([0, T])}$, thus $\left\{v^{m}(T)\right\}_{m} \subset L^{1}(\mathbb{R})$ is a Cauchy sequence, and,

$$
\lim _{m \rightarrow \infty} v^{m}(T)=v_{\text {orm }}(T) \in L^{1}(\mathbb{R}) .
$$

Moreover, by 4.39, 4.32 and a telescoping sum argument we obtain

$$
\left\|v_{\mathrm{orm}}(T)-v^{m}(T)\right\|_{L^{1}(\mathbb{R})} \leq 2\left\|f^{\prime}\right\|_{\infty}\left|u_{0}\right|_{\mathrm{BV}(\mathbb{R})}\left(|\operatorname{orm}[z]|_{\mathrm{BV}([0, T])}-\sum_{k=0}^{\ell_{m}}\left|\Delta y_{k}^{m}\right|\right) .
$$

Inequality 4.31 can be proved using a similar telescoping sum argument.

It remains to verify that $v_{\text {orm }}(T)$, under some assumptions, is equal to the unique pathwise entropy solution of (1.5) at final time $t=T$. For strictly convex fluxes $f$ and driving paths $z \in C_{0}([0, T])$ with orm $[z] \in B V([0, T])$, Theorem 4.10 below provides an error bound for approximations of pathwise entropy solutions which is different from 2.3 and shows a connection between the driving path $z$ and $\operatorname{orm}[z]$. If one were to consider an extension of $[1.5$ with orm $[z]$ given as the input, then Theorem 4.10 could serve as a basis for constructing numerical methods for $v\left(T ; \operatorname{orm}[z],\left\{\tau_{k}\right\}_{k=0}^{m}\right)$. This would differ from the methods we propose in this work, which compute approximations to $v\left(T ; z^{m},\left\{\tau_{k}\right\}_{k=0}^{m}\right)$ or $v\left(T ; \overline{\mathrm{orm}}_{m}[z],\left\{\tau_{k}\right\}_{k=0}^{m}\right)$.

Theorem 4.10. Let $u$ denote the unique pathwise entropy solution of $\sqrt{1.5}$ with initial data $u_{0} \in\left(L^{1} \cap B V\right)(\mathbb{R})$, strictly convex flux $f \in C^{2}(\mathbb{R})$ and driving path $z \in C_{0}([0, T])$. Assume that $|\operatorname{orm}[z]|_{\mathrm{BV}(\mathbb{R})}<\infty$ and for some $m \geq 2$, let $\left\{\tau_{k}\right\}_{k=0}^{m}$ denote a mesh satisfying $0=\tau_{0}<\tau_{1}<\ldots<\tau_{m}=T$. Then

$$
\begin{aligned}
\| u(T)-v & \left(T ; \operatorname{orm}[z],\left\{\tau_{k}\right\}_{k=0}^{m}\right) \|_{L^{1}(\mathbb{R})} \\
& \leq 2\left\|f^{\prime}\right\|_{\infty}\left|u_{0}\right|_{\mathrm{BV}(\mathbb{R})}\left(|\operatorname{orm}[z]|_{\mathrm{BV}([0, T])}-\mathrm{TV}\left(\operatorname{orm}[z] ;\left\{\tau_{k}\right\}_{k=0}^{m}\right)\right),
\end{aligned}
$$

where $\operatorname{TV}\left(\operatorname{orm}[z] ;\left\{\tau_{k}\right\}_{k=0}^{m}\right)$ denotes the total variation of orm $[z]$ restricted to $\left\{\tau_{k}\right\}_{k=0}^{m}$, cf. Definition 4.6.

Proof. Since $|\operatorname{orm}[z]|_{B V([0, T])}<\infty$, there is a sequence of meshes $\left\{\left\{\tau_{k}^{r}\right\}_{k=1}^{\ell_{r}}\right\}_{r=0}^{\infty}$ such that

$$
\lim _{r \rightarrow \infty} \operatorname{TV}\left(\operatorname{orm}[z] ;\left\{\tau_{k}^{r}\right\}_{k=0}^{\ell_{r}}\right)=|\operatorname{orm}[z]|_{\mathrm{BV}([0, T])} .
$$


If necessary, we can add mesh points to get nested meshes $\left\{\left\{\hat{\tau}_{k}^{r}\right\}_{k=0}^{\hat{\ell}_{r}}\right\}_{r=0}^{\infty}$ fulfilling that $\left\{\hat{\tau}_{k}^{0}\right\}_{k=0}^{\hat{\ell}_{0}}=\left\{\tau_{k}\right\}_{k=0}^{m}, \hat{\ell}_{r}<\hat{\ell}_{r+1}$ for all $r \geq 0$,

$$
\lim _{r \rightarrow \infty} \max _{k \in\left\{0,1, \ldots, \ell_{m}-1\right\}} \hat{\tau}_{k+1}^{r}-\hat{\tau}_{k}^{r}=0,
$$

and

$$
\lim _{r \rightarrow \infty} \operatorname{TV}\left(\operatorname{orm}[z] ;\left\{\hat{\tau}_{k}^{r}\right\}_{k=0}^{\hat{\ell}_{r}}\right)=|\operatorname{orm}[z]|_{B V([0, T])} .
$$

Let

$$
A_{r}^{ \pm}[z]:=\bar{A}^{+}[z]\left(\left\{\hat{\tau}_{k}^{r}\right\}_{k=0}^{\hat{\ell}_{r}}\right) \cup \bar{A}^{-}[z]\left(\left\{\hat{\tau}_{k}^{r}\right\}_{k=0}^{\hat{\ell}_{r}}\right), \quad r \geq 0 .
$$

As $A_{r}^{ \pm}[z] \subset A_{r+1}^{ \pm}[z]$ for all $r \geq 0$, we may construct a new sequence of nested meshes $\left\{\left\{\tau_{k}^{r}\right\}_{k=0}^{\ell_{r}}\right\}_{r=0}^{\infty}$ defined by

$$
\left\{\tau_{k}^{r}\right\}_{k=0}^{\ell_{r}}= \begin{cases}\left\{\hat{\tau}_{k}^{0}\right\}_{k=0}^{\hat{\ell}_{0}}=\left\{\tau_{k}\right\}_{k=0}^{m}, & \text { if } r=0, \\ \left\{\hat{\tau}_{k}^{r-1}\right\}_{k=0}^{\hat{\ell}_{r-1}} \cup A_{r-1}^{ \pm}[z], & \text { if } r \geq 1 .\end{cases}
$$

Since $\left\{\tau_{k}^{r}\right\}_{k=0}^{\ell_{r}} \supset\left\{\hat{\tau}_{k}^{r}\right\}_{k=0}^{\hat{\ell}_{r}}$ for all $r \geq 0$, it also holds that

$$
\lim _{r \rightarrow \infty} \max _{k \in\left\{0,1, \ldots, \ell_{r}-1\right\}} \tau_{k+1}^{r}-\tau_{k}^{r}=0,
$$

and

$$
\lim _{r \rightarrow \infty} \operatorname{TV}\left(\operatorname{orm}[z] ;\left\{\tau_{k}^{r}\right\}_{k=0}^{\ell_{r}}\right)=|\operatorname{orm}[z]|_{B V([0, T])} .
$$

For any $r \geq 1$, we have that

$$
\begin{aligned}
& \left\|v\left(T ; \operatorname{orm}[z],\left\{\tau_{k}\right\}_{k=0}^{m}\right)-u(T)\right\|_{L^{1}(\mathbb{R})} \\
& \leq\left\|v\left(T ; \operatorname{orm}[z],\left\{\tau_{k}\right\}_{k=0}^{m}\right)-v\left(T ; \operatorname{orm}[z],\left\{\tau_{k}^{r}\right\}_{k=0}^{\ell_{r}}\right)\right\|_{L^{1}(\mathbb{R})} \\
& \quad \quad+\left\|v\left(T ; \operatorname{orm}[z],\left\{\tau_{k}^{r}\right\}_{k=0}^{\ell_{r}}\right)-u(T)\right\|_{L^{1}(\mathbb{R})} \\
& \quad=\mathrm{I}_{r}+\mathrm{II}_{r} .
\end{aligned}
$$

Lemma 4.9 implies that for any pair of nested meshes $\left\{\tau_{k}\right\}_{k=0}^{m} \subset\left\{\tau_{k}^{r}\right\}_{k=0}^{\ell_{r}}$,

$$
\begin{aligned}
\mathrm{I}_{r} & \leq 2\left\|f^{\prime}\right\|_{\infty}\left|u_{0}\right|_{\mathrm{BV}(\mathbb{R})}\left(\operatorname{TV}\left(\operatorname{orm}[z] ;\left\{\tau_{k}^{r}\right\}_{k=0}^{\ell_{r}}\right)-\operatorname{TV}\left(\operatorname{orm}[z] ;\left\{\tau_{k}\right\}_{k=0}^{m}\right)\right) \\
& \leq 2\left\|f^{\prime}\right\|_{\infty}\left|u_{0}\right|_{\mathrm{BV}(\mathbb{R})}\left(|\operatorname{orm}[z]|_{B V([0, T])}-\operatorname{TV}\left(\operatorname{orm}[z] ;\left\{\tau_{k}\right\}_{k=0}^{m}\right)\right) .
\end{aligned}
$$

To bound $\mathrm{II}_{r}$, note first that by 4.24,

$$
\operatorname{orm}[z]\left(\tau_{k}^{r}\right)= \begin{cases}z\left(\max \left(A^{+}[z], A^{-}[z]\right)\left(\tau_{k}^{r}\right)\right) & \text { if } \quad k \in\left\{0,1, \ldots, \ell_{r}-1\right\} \\ z(T)=z\left(\tau_{\ell_{r}}^{r}\right) & \text { if } \quad k=\ell_{r} .\end{cases}
$$

Recalling that $\overline{\mathcal{S}}(0)=I$ and that $\max \left(\bar{A}^{+}[z], \bar{A}^{-}[z]\right)(\cdot)$ is a monotonically increasing function,

$$
\begin{aligned}
& v\left(T ; \operatorname{orm}[z],\left\{\tau_{k}^{r}\right\}_{k=0}^{\ell_{r}}\right) \\
& =\overline{\mathcal{S}}\left(\operatorname{orm}[z]\left(\tau_{\ell_{r}}^{r}\right)-\operatorname{orm}[z]\left(\tau_{\ell_{r}-1}^{r}\right)\right) \cdots \overline{\mathcal{S}}\left(\operatorname{orm}[z]\left(\tau_{1}^{r}\right)-\operatorname{orm}[z]\left(\tau_{0}^{r}\right)\right) u_{0} \\
& =v\left(T ; z, \max \left(A^{+}[z], A^{-}[z]\right)\left(\left\{\tau_{k}^{r}\right\}_{k=0}^{\ell_{r}}\right) \cup\{T\}\right) .
\end{aligned}
$$

By 4.17,

and 4.41 implies that

$$
\max \left\{\bar{A}^{+}[z], \bar{A}^{-}[z]\right\}\left(A_{r}^{ \pm}[z]\right)=A_{r}^{ \pm}[z],
$$

$$
\begin{aligned}
\max \left(\bar{A}^{+}[z], \bar{A}^{-}\right. & {[z])\left(\left\{\tau_{k}^{r}\right\}_{k=0}^{\ell_{r}}\right)=\max \left(\bar{A}^{+}[z], \bar{A}^{-}[z]\right)\left(A_{r}^{ \pm}[z] \cup\left\{\hat{\tau}_{k}^{r}\right\}_{k=0}^{\hat{\ell}_{r}}\right) } \\
& =\max \left(\bar{A}^{+}[z], \bar{A}^{-}[z]\right)\left(A_{r}^{ \pm}[z]\right) \cup \max \left(\bar{A}^{+}[z], \bar{A}^{-}[z]\right)\left(\left\{\hat{\tau}_{k}^{r}\right\}_{k=0}^{\hat{\ell}_{r}}\right) \\
& =A_{r}^{ \pm}[z] .
\end{aligned}
$$


Consequently,

$$
\begin{aligned}
v\left(T ; z, \max \left(A^{+}[z], A^{-}[z]\right)\left(\left\{\tau_{k}^{r}\right\}_{k=0}^{\ell_{r}}\right) \cup\{T\}\right) & =v\left(T ; z, A_{r}^{ \pm}[z] \cup\{T\}\right) \\
& =v\left(T ; \tilde{z}^{r}, A_{r}^{ \pm}[z] \cup\{T\}\right),
\end{aligned}
$$

where $\tilde{z}^{r}:=\mathcal{I}[z]\left(\cdot ; A_{r}^{ \pm}[z] \cup\{T\}\right)$. Introducing the function $z^{r}=\mathcal{I}[z]\left(\cdot ;\left\{\tau_{k}^{r}\right\}_{k=0}^{\ell_{r}}\right)$, we may bound the second term as follows

$$
\begin{aligned}
\mathrm{II}_{r} & \leq\left\|u(T)-v\left(T ; z^{r},\left\{\tau_{k}^{r}\right\}_{k=0}^{\ell_{r}}\right)\right\|_{1}+\left\|v\left(T ; z^{r},\left\{\tau_{k}^{r}\right\}_{k=0}^{\ell_{r}}\right)-v\left(T ; \tilde{z}^{r}, A_{r}^{ \pm}[z] \cup\{T\}\right)\right\|_{1} \\
& =: \mathrm{II}_{r, 1}+\mathrm{II}_{r, 2} .
\end{aligned}
$$

Theorem 2.1 and the property $z^{r}(T)=z(T)$ for all $r \geq 1$ imply that there exists a constant $C>0$ that is uniform in $r \geq 1$ such that

$$
\mathrm{II}_{r, 1} \leq C \sqrt{\max _{s \in[0, T]}\left|z-z^{r}\right|(s)}, \quad \forall r \geq 1 .
$$

Since $z$ is uniformly continuous on $[0, T]$, it follows from 4.42 that

$$
\lim _{r \rightarrow \infty} \mathrm{II}_{r, 1}=0 \text {. }
$$

The term $\mathrm{II}_{r, 2}$ is bounded by verifying that

$$
v\left(T ; z^{r},\left\{\tau_{k}^{r}\right\}_{k=0}^{\ell_{r}}\right)=v\left(T ; \tilde{z}^{r}, A_{r}^{ \pm}[z] \cup\{T\}\right),
$$

which actually means that $\mathrm{II}_{r, 2}=0$. Note first that since $A_{r}^{ \pm}[z] \cup\{T\} \subset\left\{\tau_{k}^{r}\right\}_{k=0}^{\ell_{r}}$, we may introduce the monotonically increasing function $h:\left\{0,1, \ldots, \ell_{r}\right\} \rightarrow\left\{0,1, \ldots, \ell_{r}\right\}$ defined by

$$
h(k)= \begin{cases}\left\{s \in\{0,1, \ldots, k\} \mid \tau_{s}^{r}=\max \left(\bar{A}^{+}[z], \bar{A}^{-}[z]\right)\left(\tau_{k}^{r}\right)\right\} & \text { if } k<\ell_{r} \\ \ell_{r} & \text { if } k=\ell_{r} .\end{cases}
$$

and write $\left\{\tau_{h(k)}\right\}_{k=0}^{\ell_{r}}=A_{r}^{ \pm}[z] \cup\{T\}$. Using this representation and that

$$
\left.\tilde{z}^{r}\right|_{A_{r}^{ \pm}[z] \cup\{T\}}=\left.z\right|_{A_{r}^{ \pm}[z] \cup\{T\}}=\left.z^{r}\right|_{A_{r}^{ \pm}[z] \cup\{T\}},
$$

we obtain

$$
v\left(T ; \tilde{z}^{r},\left\{\tau_{h(k)}\right\}_{k=0}^{\ell_{r}}\right)=\overline{\mathcal{S}}\left(z^{r}\left(\tau_{h\left(\ell_{r}\right)}^{r}\right)-z^{r}\left(\tau_{h\left(\ell_{r}-1\right)}^{r}\right)\right) \cdots \overline{\mathcal{S}}\left(z^{r}\left(\tau_{h(1)}^{r}\right)-z^{r}\left(\tau_{h(0)}^{r}\right)\right) u_{0}
$$

Recalling that

$$
v\left(T ; z^{r},\left\{\tau_{k}^{r}\right\}_{k=0}^{\ell_{r}}\right)=\overline{\mathcal{S}}\left(z^{r}\left(\tau_{\ell_{r}}\right)-z^{r}\left(\tau_{\ell_{r}-1}\right)\right) \cdots \overline{\mathcal{S}}\left(z^{r}\left(\tau_{1}\right)-z^{r}\left(\tau_{0}\right)\right) u_{0},
$$

equality 4.48 follows by a straightforward induction argument if the following equality holds for all $k \in\left\{0,1, \ldots, \ell_{r}-1\right\}$ such that $h(k+1)-h(k) \geq 2$ :

$$
\begin{aligned}
& \overline{\mathcal{S}}\left(z^{r}\left(\tau_{h(k+1)}^{r}\right)-z^{r}\left(\tau_{h(k+1)-1}^{r}\right)\right) \cdots \overline{\mathcal{S}}\left(z^{r}\left(\tau_{h(k)+1}^{r}\right)-z^{r}\left(\tau_{h(k)}^{r}\right)\right) v\left(\tau_{h(k)}^{r} ; z^{r},\left\{\tau_{h(k)}^{r}\right\}_{k=0}^{\ell_{r}}\right) \\
& =\overline{\mathcal{S}}\left(z^{r}\left(\tau_{h(k+1)}^{r}\right)-z^{r}\left(\tau_{h(k)}^{r}\right)\right) v\left(\tau_{h(k)}^{r} ; z^{r},\left\{\tau_{h(k)}^{r}\right\}_{k=0}^{\ell_{r}}\right) .
\end{aligned}
$$

Assume $k \in\left\{0,1, \ldots, \ell_{r}-1\right\}$ is such that $h(k+1)-h(k) \geq 2$. Then

$$
\left\{\bar{A}^{-}[z]\left(\tau_{h(k+1)-1}^{r}\right), \bar{A}^{+}[z]\left(\tau_{h(k+1)-1}^{r}\right)\right\} \subset A_{r}^{ \pm}[z]
$$

and

$$
\max \left(\bar{A}^{-}[z], \bar{A}^{+}[z]\right)\left(\tau_{h(k+1)-1}^{r}\right)=\tau_{h(k)}^{r} .
$$

This implies that for all $s \in\{h(k), \ldots, h(k+1)-1\}$,

$$
\begin{gathered}
z^{r}\left(\tau_{s}^{r}\right) \geq M^{-}\left[z^{r}\right]\left(\tau_{h(k+1)-1}^{r}\right) \geq M^{-}[z]\left(\tau_{h(k+1)-1}^{r}\right) \\
=z\left(\bar{A}^{-}[z]\left(\tau_{h(k+1)-1}^{r}\right)\right) \geq M^{-}\left[z^{r}\right]\left(\tau_{h(k)}^{r}\right)
\end{gathered}
$$


and

Consequently,

$$
\begin{gathered}
z^{r}\left(\tau_{s}^{r}\right) \leq M^{+}\left[z^{r}\right]\left(\tau_{h(k+1)-1}^{r}\right) \leq M^{+}[z]\left(\tau_{h(k+1)-1}^{r}\right) \\
=z\left(\bar{A}^{+}[z]\left(\tau_{h(k+1)-1}^{r}\right)\right) \leq M^{+}\left[z^{r}\right]\left(\tau_{h(k)}^{r}\right) .
\end{gathered}
$$

$$
z^{r}\left(\left[\tau_{h(k)}, \tau_{h(k+1)-1}\right]\right) \subset\left[M^{-}\left[z^{r}\right]\left(\tau_{h(k)}^{r}\right), M^{+}\left[z^{r}\right]\left(\tau_{h(k)}^{r}\right)\right] .
$$

Consider the following three cases: $z^{r}\left(\tau_{h(k+1)}^{r}\right)-z^{r}\left(\tau_{h(k+1)-1}^{r}\right)=0, z^{r}\left(\tau_{h(k+1)}^{r}\right)-$ $z^{r}\left(\tau_{h(k+1)-1}^{r}\right)>0$, and $z^{r}\left(\tau_{h(k+1)}^{r}\right)-z^{r}\left(\tau_{h(k+1)-1}^{r}\right)<0$.

If $z^{r}\left(\tau_{h(k+1)}^{r}\right)-z^{r}\left(\tau_{h(k+1)-1}^{r}\right)=0$, then

$$
z^{r}\left(\left[\tau_{h(k)}, \tau_{h(k+1)}\right]\right) \subset\left[M^{-}\left[z^{r}\right]\left(\tau_{h(k)}^{r}\right), M^{+}[z]\left(\tau_{h(k)}^{r}\right)\right],
$$

and 4.49 follows from Lemma 4.3 .

If $z^{r}\left(\tau_{h(k+1)}^{r}\right)-z^{r}\left(\tau_{h(k+1)-1}^{r}\right)>0$, then, since $\tau_{h(k+1)} \in A_{r}^{ \pm}[z] \cup\{T\}$, either $\tau_{h(k+1)}=T \notin A_{r}^{ \pm}[z]$ or $\tau_{h(k+1)} \in A_{r}^{ \pm}[z]$. If $\tau_{h(k+1)}=T \notin A_{r}^{ \pm}[z]$, then $\max \left(\bar{A}^{+}[z], \bar{A}^{-}[z]\right)(T)=$ $\tau_{h\left(\ell_{r}-1\right)}^{r}$, and

$$
\begin{aligned}
& z^{r}\left(\tau_{h\left(\ell_{r}\right)}\right) \geq z\left(\bar{A}^{-}[z]\left(\tau_{h\left(\ell_{r}\right)}^{r}\right)\right) \geq M^{-}\left[z^{r}\right]\left(\tau_{h\left(\ell_{r}-1\right)}^{r}\right), \\
& z^{r}\left(\tau_{h\left(\ell_{r}\right)}\right) \leq z\left(\bar{A}^{+}[z]\left(\tau_{h\left(\ell_{r}\right)}^{r}\right)\right) \leq M^{+}\left[z^{r}\right]\left(\tau_{h\left(\ell_{r}-1\right)}^{r}\right) .
\end{aligned}
$$

Hence 4.51 holds and 4.49 follows. If $z^{r}\left(\tau_{h(k+1)}^{r}\right)-z^{r}\left(\tau_{h(k+1)-1}^{r}\right)>0$ and $\tau_{h(k+1)}^{r} \in A_{r}^{ \pm}[z]$, then

$$
\begin{aligned}
z\left(\tau_{h(k+1)}^{r}\right) & =z^{r}\left(\tau_{h(k+1)-1}^{r}\right)+z^{r}\left(\tau_{h(k+1)}^{r}\right)-z^{r}\left(\tau_{h(k+1)-1}^{r}\right) \\
& \geq z\left(\bar{A}^{-}[z]\left(\tau_{h(k+1)-1}^{r}\right)\right)+z^{r}\left(\tau_{h(k+1)}^{r}\right)-z^{r}\left(\tau_{h(k+1)-1}^{r}\right) \\
& \geq M^{-}[z]\left(\tau_{h(k)}^{r}\right)+z^{r}\left(\tau_{h(k+1)-1}^{r}\right)-z^{r}\left(\tau_{h(k+1)-1}^{r}\right) \\
& >M^{-}[z]\left(\tau_{h(k)}^{r}\right) .
\end{aligned}
$$

This implies that $\tau_{h(k+1)}^{r}=\bar{A}^{+}[z]\left(\tau_{h(k+1)}^{r}\right)$ and we conclude from

$$
M^{+}\left[z^{r}\right]\left(\tau_{h(k+1)}^{r}\right) \leq M^{+}[z]\left(\tau_{h(k+1)}^{r}\right)=z\left(\tau_{h(k+1)}^{r}\right)=z^{r}\left(\tau_{h(k+1)}^{r}\right)
$$

that $M^{+}\left[z^{r}\right]\left(\tau_{h(k+1)}^{r}\right)=z^{r}\left(\tau_{h(k+1)}^{r}\right)$. Moreover, by 4.50 and $\dot{z}^{r}\left(\tau_{h(k+1)-1}^{r}+\right)>0$, there exists a unique $\tau \in\left[\tau_{h(k+1)-1}^{r}, \tau_{h(k+1)}^{r}\right]$ such that

$$
z^{r}(\tau)=M^{+}\left[z^{r}\right]\left(\tau_{h(k)}^{r}\right) \quad \text { and } \bar{A}^{+}[z](\tau)=\tau .
$$

Consequently,

$$
z^{r}\left(\left[\tau_{h(k)}, \tau\right]\right) \subset\left[M^{-}\left[z^{r}\right]\left(\tau_{h(k)}^{r}\right), M^{+}\left[z^{r}\right]\left(\tau_{h(k)}^{r}\right)\right],
$$

and Lemma 4.3 yields

$$
\begin{aligned}
& \overline{\mathcal{S}}\left(z^{r}\left(\tau_{h(k+1)}^{r}\right)-z^{r}\left(\tau_{h(k+1)-1}^{r}\right)\right) \cdots \overline{\mathcal{S}}\left(z^{r}\left(\tau_{h(k)+1}^{r}\right)-z^{r}\left(\tau_{h(k)}^{r}\right)\right) v\left(\tau_{h(k)}^{r} ; z^{r},\left\{\tau_{h(k)}^{r}\right\}_{k=0}^{\ell_{r}}\right) \\
& =\overline{\mathcal{S}}\left(z^{r}\left(\tau_{h(k+1)}^{r}\right)-z^{r}(\tau)\right) \mathcal{S}\left(z^{r}(\tau)-z^{r}\left(\tau_{h(k)}^{r}\right)\right) v\left(\tau_{h(k)}^{r} ; z^{r},\left\{\tau_{h(k)}^{r}\right\}_{k=0}^{\ell_{r}}\right) \\
& =\overline{\mathcal{S}}\left(z^{r}\left(\tau_{h(k+1)}^{r}\right)-z^{r}\left(\tau_{h(k)}^{r}\right)\right) v\left(\tau_{h(k)}^{r} ; z^{r},\left\{\tau_{h(k)}^{r}\right\}_{k=0}^{\ell_{r}}\right),
\end{aligned}
$$

where the last equality follows from

$$
\begin{aligned}
& \left(z^{r}\left(\tau_{h(k+1)}^{r}\right)-z^{r}(\tau)\right)\left(z^{r}(\tau)-z^{r}\left(\tau_{h(k)}^{r}\right)\right) \\
& \quad=\left(M^{+}\left[z^{r}\right]\left(\tau_{h(k+1)}^{r}\right)-M^{+}\left[z^{r}\right]\left(\tau_{h(k)}^{r}\right)\right)\left(M^{+}\left[z^{r}\right]\left(\tau_{h(k)}^{r}\right)-z^{r}\left(\tau_{h(k)}^{r}\right)\right) \geq 0
\end{aligned}
$$

and the argument preceding 4.35.

Verifying 4.49 for the case $z^{r}\left(\tau_{h(k+1)}^{r}\right)-z^{r}\left(\tau_{h(k+1)-1}^{r}\right)<0$ may be done similarly. This yields

$$
\mathrm{II}_{r, 2}=0 \quad \forall r \geq 1,
$$


and by 4.43), 4.44, 4.46) and 4.47),

$$
\begin{aligned}
& \left\|v\left(T ; \operatorname{orm}[z],\left\{\tau_{k}\right\}_{k=0}^{m}\right)-u(T)\right\|_{1} \leq \lim _{r \rightarrow \infty}\left(\mathrm{I}_{r}+\mathrm{II}_{r, 2}\right) \\
& \quad \leq 2\left\|f^{\prime}\right\|_{\infty}\left|u_{0}\right|_{\mathrm{BV}(\mathbb{R})}\left(|\operatorname{orm}[z]|_{B V(\mathbb{R})}-\mathrm{TV}\left(\operatorname{orm}[z] ;\left\{\tau_{k}\right\}_{k=0}^{m}\right)\right) .
\end{aligned}
$$

\section{IMPROVED NUMERICAL METHOD}

In this section we make use of the $\overline{\mathrm{orm}}_{m}[\cdot]$ operator to construct an improved numerical method for 2.1) with strictly convex fluxes $f$. To minimize the number of interpolation points in the driving path, thereby improving the efficiency of the method, we first describe a procedure for removing Lemma 4.3 type (i) and (ii) "oscillatory cancellations" from paths of the form $\operatorname{orm}_{m}[z]$, cf. Section 5.1. The improved method is described in Section 5.2. The main result, Theorem 5.2 reveals that the possible efficiency gain is linked to the ratio $\left|\overline{\mathrm{orm}}_{m}[z]\right|_{\mathrm{BV}([0, T])} /\left|z^{m}\right|_{\mathrm{BV}([0, T])}$. To verify that the efficiency gain is relevant for an important class of driving paths, Section 5.3 shows that for a standard Wiener path $z,|\operatorname{orm}[z]|_{\mathrm{BV}([0, T])}<\infty$ almost surely, whereas $|z|_{\mathrm{BV}([0, T])}=\infty$. Consequently, the improved method is asymptotically orders of magnitude more efficient than the original method. Section 5.4 presents numerical comparisons of the various methods and a numerical study of the mean of $\left|\overline{\mathrm{orm}}_{m}[z]\right|_{\mathrm{BV}([0, T])}$ and $\left|z^{m}\right|_{\mathrm{BV}([0, T])}$ for a class of fractional Brownian motions $z$.

5.1. Removal of Lemma 4.3 type (i), (ii) cancellations. Theorem 4.5 shows that for strictly convex fluxes $f$ and paths $z \in C_{0}([0, T])$, the entropy solution of (2.1) at time $t=T$ with driving path $z^{m}=\mathcal{I}^{m}[z]$ can be found by replacing $z^{m}$ with $y^{m}=\overline{\mathrm{orm}}_{m}[z]$ and computing $v\left(T ; y^{m},\left\{\tau_{j(k)}\right\}_{k=0}^{\bar{m}}\right)$, cf. (4.21), or equivalently using $\operatorname{orm}\left[z^{m}\right]$, cf. Theorem 4.7. One may view $\overline{\operatorname{orm}}_{m}[z]$ as a version of $z^{m}$ with Lemma 4.3 type (iii) "oscillatory cancellations" removed. A further implication of the lemma is that we may also remove Lemma 4.3 type (i-ii) "oscillatory cancellations" from $\overline{\mathrm{orm}}_{m}[z]$ and still preserve the entropy solution at final time $t=T$. The following algorithm describes the removal procedure:

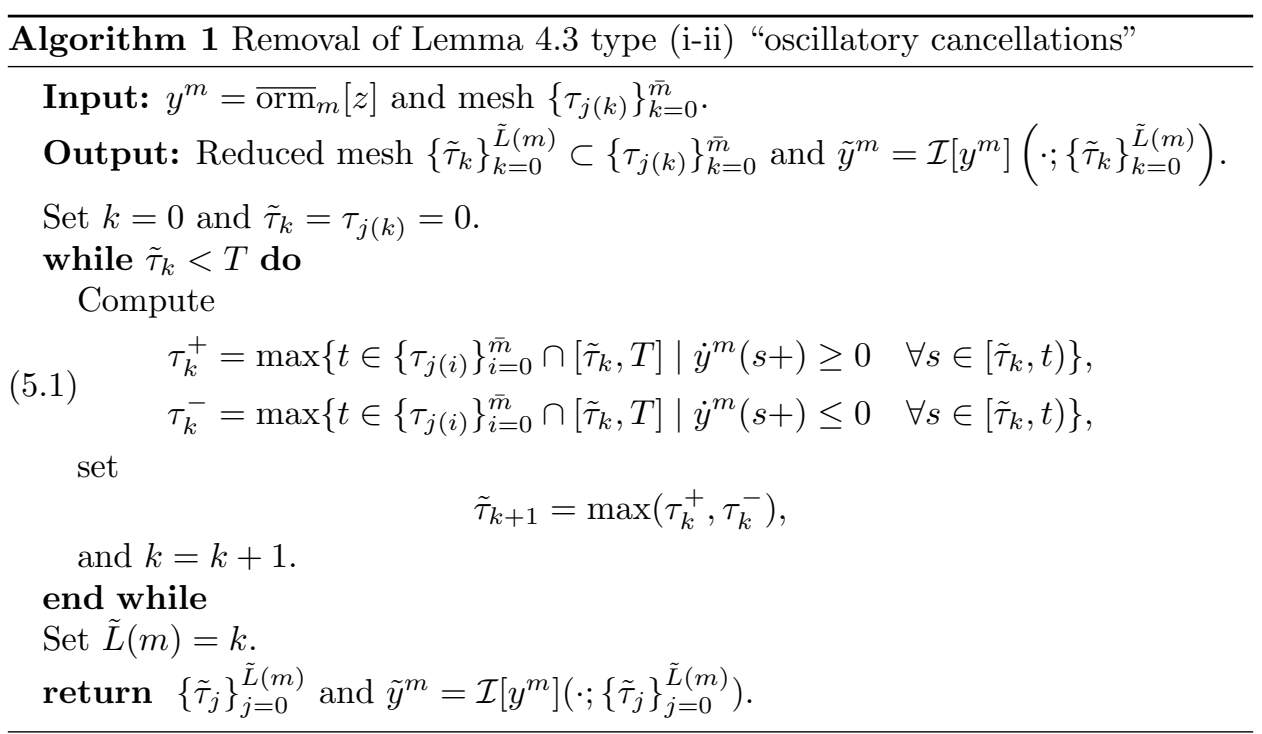

Figure 10 illustrates the transition from $y^{m}=\overline{\mathrm{orm}}_{m}[z]$ to $\tilde{y}^{m}$ computed by Algorithm 1 

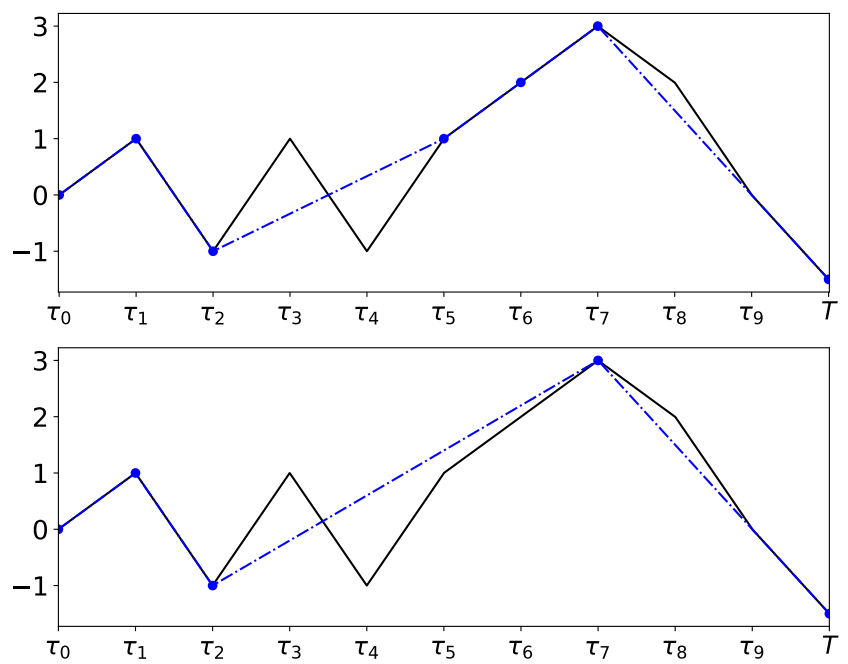

FiguRE 10. Top: The piecewise linear path $z^{m}$ with $m=10$ (black line) and the associated $y^{m}=\overline{\operatorname{orm}}_{m}[z]$ (blue dash-dotted line). Blue dots mark the value of $\overline{\mathrm{orm}}_{m}[z]$ at its interpolation points $\left\{\tau_{j(k)}\right\}_{k=0}^{\bar{m}}$. Bottom: The piecewise linear path $z^{m}$ (black line) and the associated $\tilde{y}^{m}$ computed by Algorithm 1 (blue dashdotted line). Blue dots mark the value of $\tilde{y}^{m}$ at its interpolation points $\left\{\tilde{\tau}_{k}\right\}_{k=0}^{\tilde{L}(m)}$.

For later reference, note that the output mesh of Algorithm 1 satisfies $\left\{\tilde{\tau}_{k}\right\}_{k=0}^{\tilde{L}(m)-1} \subset$ $A^{ \pm}\left[z^{m}\right]$, and, using that for all $k<\tilde{L}(m)$,

$$
y^{m}\left(\bar{A}^{+}\left[z^{m}\right]\left(\tilde{\tau}_{k}\right)\right)=M^{+}\left[z^{m}\right]\left(\tilde{\tau}_{k}\right) \quad \text { and } \quad y^{m}\left(\bar{A}^{-}\left[z^{m}\right]\left(\tilde{\tau}_{k}\right)\right)=M^{-}\left[z^{m}\right]\left(\tilde{\tau}_{k}\right),
$$

cf. Definition 4.15) and 4.16), it follows that $\left\{y^{m}\left(\bar{A}^{+}\left[z^{m}\right]\left(\tilde{\tau}_{k}\right)\right)\right\}_{k=0}^{\tilde{L}(m)-1}$ and $\left\{y^{m}\left(\bar{A}^{-}\left[z^{m}\right]\left(\tilde{\tau}_{k}\right)\right)\right\}_{k=0}^{L(m)-1}$ respectively are monotonically increasing and decreasing sequences. For any $1 \leq k \leq \tilde{L}(m)-2$ such that $\tilde{\tau}_{k}=\bar{A}^{+}\left[z^{m}\right]\left(\tilde{\tau}_{k}\right)$, it must hold that $\dot{y}^{m}\left(\tilde{\tau}_{k}+\right)<0$. Consequently, $\tilde{\tau}_{k+1}=\tau_{k}^{-}$, and since

$$
y^{m}\left(\tilde{\tau}_{k+1}\right)<y^{m}\left(\tilde{\tau}_{k}\right) \leq y^{m}\left(\bar{A}^{+}\left[z^{m}\right]\left(\tilde{\tau}_{k+1}\right)\right)
$$

implies that $\tilde{\tau}_{k+1} \neq \bar{A}^{+}\left[z^{m}\right]\left(\tilde{\tau}_{k+1}\right)$, it must hold that $\tilde{\tau}_{k+1}=\bar{A}^{-}\left[z^{m}\right]\left(\tilde{\tau}_{k+1}\right)$. By similar reasoning, if $1 \leq k \leq \tilde{L}(m)-2$ is such that $\tilde{\tau}_{k}=\bar{A}^{-}\left[z^{m}\right]\left(\tilde{\tau}_{k}\right)$, then $\tilde{\tau}_{k+1}=$ $\bar{A}^{+}\left[z^{m}\right]\left(\tilde{\tau}_{k}\right)$. We obtain that $\tilde{y}_{k}^{m} \tilde{y}_{k+1}^{m} \leq 0$ for all $k \leq \tilde{L}(m)-2$,

$$
\tilde{y}_{k}^{m} \tilde{y}_{k+2}^{m} \geq 0 \quad \text { and } \quad\left|\tilde{y}_{k}^{m}\right| \leq\left|\tilde{y}_{k+2}^{m}\right| \quad \forall k \leq \tilde{L}(m)-3,
$$

and as $\tilde{y}_{1}^{m} \neq 0$, if $\tilde{L}(m)>1$,

$$
\Delta \tilde{y}_{k}^{m} \Delta \tilde{y}_{k+1}^{m}<0 \quad \text { and } \quad\left|\Delta \tilde{y}_{k}^{m}\right| \leq\left|\Delta \tilde{y}_{k+1}^{m}\right| \quad \forall k \leq \tilde{L}(m)-3 .
$$

(For the last index, $k=L(m)-2$, the properties 5.2 hold if $T \in A^{ \pm}\left[z^{m}\right]$, but may not hold if $T \notin A^{ \pm}\left[z^{m}\right]$.)

Lemma 5.1. Assume $f \in C^{2}(\mathbb{R})$ is strictly convex, $u_{0} \in\left(L^{1} \cap B V\right)(\mathbb{R})$ and $z \in$ $C_{0}([0, T])$. For any mesh $0=\tau_{0}<\tau_{1}<\ldots<\tau_{m}=T, m \geq 2$, let $y^{m}=\overline{\operatorname{orm}}_{m}[z]$ and $\tilde{y}^{m}=\mathcal{I}\left[y^{m}\right]\left(\cdot ;\left\{\tilde{\tau}_{k}\right\}_{k=0}^{\tilde{L}(m)}\right)$, cf. Algorithm 1 .

Then

$$
v\left(T ; y^{m},\left\{\tau_{k}\right\}_{k=0}^{m}\right)=v\left(T ; \tilde{y}^{m},\left\{\tilde{\tau}_{k}\right\}_{k=0}^{\tilde{L}(m)}\right)
$$


and

$$
\left|y^{m}\right|_{\mathrm{BV}([0, T])}=\left|\tilde{y}^{m}\right|_{\mathrm{BV}([0, T])} .
$$

Proof. Equation (5.3) follows directly by Lemma 4.3

To verify (5.4), note by Algorithm 1 that for any $k \in\{0,1, \ldots, \tilde{L}(m)-1\}$, it either holds that

$$
\dot{y}^{m}(s+) \geq 0 \quad \text { and } \quad \dot{\tilde{y}}^{m}(s+) \geq 0 \quad \forall s \in\left[\tilde{\tau}_{k}, \tilde{\tau}_{k+1}\right),
$$

or

$$
\dot{y}^{m}(s+) \leq 0 \quad \text { and } \quad \dot{\tilde{y}}^{m}(s+)<0 \quad \forall s \in\left[\tilde{\tau}_{k}, \tilde{\tau}_{k+1}\right),
$$

and it holds that

$$
\tilde{y}^{m}\left(\tilde{\tau}_{k}\right)=y^{m}\left(\tilde{\tau}_{k}\right) \quad \forall k \in\{0,1, \ldots, \tilde{L}(m)\}
$$

Consequently,

$$
\begin{aligned}
\left|y^{m}\right|_{\mathrm{BV}([0, T])} & =\sum_{k=0}^{\bar{m}-1}\left|y^{m}\left(\tau_{j(k+1)}\right)-y^{m}\left(\tau_{j(k)}\right)\right| \\
& =\sum_{k=0}^{\tilde{L}(m)-1}\left|y^{m}\left(\tilde{\tau}_{k+1}\right)-y^{m}\left(\tilde{\tau}_{k}\right)\right|=\left|\tilde{y}^{m}\right|_{\mathrm{BV}([0, T])}
\end{aligned}
$$

5.2. Orm based numerical method. We now propose a numerical method that makes use of the piecewise linear orm function of $z$ and $\tilde{y}^{m}$ to compute entropy solutions at final time:

(i) Approximate the driving path $z \in C_{0}([0, T])$ by the piecewise linear interpolant $z^{m}=\mathcal{I}^{m}[z]$ on a uniform mesh $\left\{\tau_{k}\right\}_{k=0}^{m}$ with step size $T / m$.

(ii) Compute $y^{m}=\overline{\operatorname{orm}}_{m}[z]$ and its interpolation points $\left\{\tau_{j(k)}\right\}_{k=0}^{\bar{m}}$, cf. Definition 4.3

(iii) Compute $\tilde{y}^{m}$ and its interpolation points $\left\{\hat{\tau}_{k=0}\right\}_{k=0}^{\hat{L}(m)}$ by Algorithm 1 with $y^{m}$ and $\left\{\tau_{j(k)}\right\}_{k=0}^{\bar{m}}$ as input.

(iv) Compute a numerical approximation of $v\left(T ; \tilde{y}^{m},\left\{\tilde{\tau}_{k}\right\}_{k=0}^{\tilde{L}(m)}\right)$, cf. 4.21, using a consistent, conservative and monotone finite volume method.

The numerical approximation of $v\left(T ; \tilde{y}^{m},\left\{\tau_{k}\right\}_{k=0}^{\tilde{L}(m)}\right)$, denoted $U(T)$, is obtained by initializing $U(0)$ using 3.5 and iteratively, for $k=0,1, \ldots, \tilde{L}(m)-1$, compute the numerical solution of

$$
\begin{aligned}
\partial_{t} \tilde{u}+\operatorname{sign}\left(\Delta \tilde{y}_{k}^{m}\right) \partial_{x} f(\tilde{u}) & =0 \quad \text { in } \quad\left(0,\left|\Delta \tilde{y}_{k}^{m}\right|\right] \times \mathbb{R}, \\
\tilde{u}(0) & =U\left(\tilde{\tau}_{k}\right),
\end{aligned}
$$

and setting $U\left(\tilde{\tau}_{k+1}\right)=\hat{\tilde{u}}\left(\left|\Delta \tilde{y}_{k}^{m}\right| ; U\left(\tilde{\tau}_{k}\right)\right)$, where $\hat{\tilde{u}}\left(s ; U\left(\tilde{\tau}_{k}\right)\right)$ denotes the numerical solution of 5.5 with $\hat{\tilde{u}}(0)=U\left(\tilde{\tau}_{k}\right)$. We let $\tilde{n}(k) \geq 1$ denote the number of uniform timesteps used in the numerical solution of $[5.5)$ over $\left[0,\left|\Delta \tilde{y}_{k}^{m}\right|\right]$, and

$$
N=\sum_{k=0}^{\tilde{L}(m)-1} \tilde{n}(k)
$$

is the total number of timesteps used to obtain the final time solution $U(T)$. The size of the uniform timesteps used in the numerical solution of the $k$-th problem $(5.5)$, for $k \in\{0,1, \ldots, \tilde{L}(m)-1\}$, is determined through the following CFL 
condition:

$$
\left\{\begin{array}{l}
\widetilde{\Delta} t_{k}=\frac{\left|\Delta \tilde{y}_{k}^{m}\right|}{\tilde{n}(k)}, \quad \text { where } \\
\tilde{n}(k)=\max \left(\left\lceil\frac{\left|\Delta \tilde{y}_{k}^{m}\right| \mid f^{\prime} \|_{\infty}}{C_{\mathrm{CFL}} \Delta x}\right\rceil, 1\right) .
\end{array}\right.
$$

In other words, the numerical solution of the $k$-th problem is computed on the temporal mesh discretization

$$
0=t_{k, 0} \leq t_{k, 1} \leq \ldots \leq t_{k, \tilde{n}(k)}=\left|\Delta \tilde{y}_{k}^{m}\right|,
$$

where $t_{k, r}=r \widetilde{\Delta} t_{k}$ for $0 \leq r \leq \tilde{n}(k)$.

For a given $z \in C_{0}^{0, \alpha}(\mathbb{R}), \alpha \in(0,1]$, Theorem 3.4 shows that the factors $\left|z^{m}\right|_{B V([0, T])}^{5}$ and $\left|z^{m}\right|_{B V([0, T])}^{6}$ respectively enter in lower and upper bounds of the computational cost of solving $v\left(T ; z^{m},\left\{\tau_{k}\right\}_{k=0}^{m}\right)$ by the adaptive time stepping method in Section 3 . In comparison, the method considered here solves $v\left(T ; \tilde{y}^{m},\left\{\tilde{\tau}_{k}\right\}_{k=0}^{\tilde{L}(m)}\right)$, and since

$$
\left|\tilde{y}^{m}\right|_{B V([0, T])}=\left|\overline{\mathrm{orm}}_{m}\left[z^{m}\right]\right|_{B V([0, T])} \leq\left|z^{m}\right|_{B V([0, T])} \quad \forall m \geq 2,
$$

cf. Lemma 5.1, it is expected that this method is more efficient. The following theorem states conditions ensuring efficiency gains.

Theorem 5.2. Let $u \in C\left([0, T] ; L^{1}(\mathbb{R})\right)$ denote the unique pathwise entropy solution of $(1.5)$ for given $u_{0} \in\left(L^{1} \cap B V\right)(\mathbb{R})$ with $\operatorname{Leb}\left(\operatorname{supp}\left(u_{0}\right)\right)>0$, strictly convex $f \in C^{2}(\mathbb{R})$ and $z \in C_{0}^{0, \alpha}([0, T])$ with $\alpha \in(0,1]$. For any $m \geq 2$, let $\left\{\tau_{j}\right\}_{j=0}^{m} \subset[0, T]$ denote the uniform mesh with step size $\Delta \tau=T / m$ and assume the computational cost of generating the interpolant $z^{m}=\mathcal{I}^{m}[z]$ is $\Theta\left(m^{\beta}\right)$ for some $\beta \geq 1$. Set $y^{m}=\overline{\operatorname{orm}}_{m}[z]$, and let $\tilde{y}^{m}=\mathcal{I}\left[y^{m}\right]\left(\cdot ;\left\{\tilde{\tau}_{k}\right\}_{k=0}^{\tilde{L}(m)}\right)$ denote the function generated by Algorithm 1 . Let $U$ denote the numerical solution described in Section 5 satisfying the local CFL condition (5.6) and with spatial resolution constraint

$$
\Delta x=\Theta\left(\frac{\Delta \tau^{\alpha}}{\max \left(\left|y^{m}\right|_{B V([0, T])}^{2}, 1\right)}\right) .
$$

Assume that the spatial support of $U([0, T])$ is covered by an interval $\left[a_{m}, b_{m}\right] \subset \mathbb{R}$ that satisfies

$$
c_{1} \leq b_{m}-a_{m} \leq c_{2}(1+N \Delta x),
$$

for some $c_{1}, c_{2}>0, c f .(3.10)$, and that at least one of the following conditions hold:

(a)

$$
\tilde{L}(m)=\mathcal{O}\left(m^{\alpha} \max \left(\left|y^{m}\right|_{B V([0, T])}^{3}, 1\right)\right),
$$

(b) there exist $\tilde{m} \geq 2$ and $\tilde{c}>0$ such that

$$
\max \left(M^{+}\left[z^{m}\right]\left(\tau_{\left\lceil m^{\alpha}\right\rceil}\right),\left|M^{-}\left[z^{m}\right]\left(\tau_{\left\lceil m^{\alpha}\right\rceil}\right)\right|\right) \geq \tilde{c} m^{-\alpha} \quad \forall m \geq \tilde{m} .
$$

Then

$$
N=\sum_{k=0}^{\tilde{L}(m)-1} \widetilde{n}(k)=\mathcal{O}\left(\frac{\max \left(\left|y^{m}\right|_{B V([0, T])}^{3}, 1\right)}{\Delta \tau^{\alpha}}\right)
$$

and

$$
\|u(T)-U(T)\|_{1}=\mathcal{O}\left(m^{-\alpha / 2}\right)
$$


is achieved at the computational cost

$$
\begin{aligned}
& \hat{c}_{1}\left(\max \left(\left|y^{m}\right|_{B V([0, T])}^{5}, 1\right) m^{2 \alpha}+m^{\beta}\right) \\
& \leq \operatorname{Cost}(U) \leq \\
& \quad \hat{c}_{2}\left(\max \left(\left|y^{m}\right|_{B V([0, T])}^{6}, 1\right) m^{2 \alpha}+m^{\beta}\right),
\end{aligned}
$$

for some $\hat{c}_{1}, \hat{c}_{2}>0$.

Proof. By the CFL condition (5.6), it holds for all $m \geq 2$ that

$$
\max _{k \in\{0,1, \ldots, \tilde{L}(m)-1\}} \widetilde{\Delta} t_{k} \leq \frac{C_{\mathrm{CFL}}}{\left\|f^{\prime}\right\|_{\infty}} \Delta x .
$$

Introducing the shorthand

$$
\tilde{v}^{m}\left(\tilde{\tau}_{k}\right):=v\left(\tilde{\tau}_{k} ; \tilde{y}^{m},\left\{\tilde{\tau}_{k}\right\}_{k=0}^{\tilde{L}(m)}\right) \quad \forall k \in\{0,1, \ldots, \tilde{L}(m)\},
$$

and using Kuznetsov's lemma, cf. [51, Example 3.15], the error of the numerical method at time $\tilde{\tau}_{k+1}$ can be bounded by

$$
\begin{aligned}
\left\|\tilde{v}^{m}\left(\tilde{\tau}_{k+1}\right)-U\left(\tilde{\tau}_{k+1}\right)\right\|_{1} & \leq\left\|\tilde{v}^{m}\left(\tilde{\tau}_{k}\right)-U\left(\tilde{\tau}_{k}\right)\right\|_{1}+C\left|\Delta \tilde{y}_{k}^{m}\right|\left(\sqrt{\widetilde{\Delta} t_{k}}+\sqrt{\Delta x}\right) \\
& \leq\left\|\tilde{v}^{m}\left(\tilde{\tau}_{k}\right)-U\left(\tilde{\tau}_{k}\right)\right\|_{1}+C\left|\Delta \tilde{y}_{i}^{m}\right| \sqrt{\Delta x}
\end{aligned}
$$

for some $C>0$ that depends on $\left\|f^{\prime}\right\|_{L^{\infty}},\left|u_{0}\right|_{B V(\mathbb{R})}$ and the numerical scheme. Using that $\tilde{v}^{m}(0)=u_{0}, \tilde{v}^{m}(T)=u^{m}(T)$ and $\left|\tilde{y}^{m}\right|_{B V([0, T])}=\left|y^{m}\right|_{B V([0, T])}$, cf. Lemma 5.1 and Theorem 4.5 we obtain

$$
\left\|u^{m}(T)-U(T)\right\|_{1} \leq\left\|u_{0}-U(0)\right\|_{1}+C\left|y^{m}\right|_{B V([0, T])} \sqrt{\Delta x} .
$$

By Theorem 2.1 (5.10), and (5.8),

$$
\begin{aligned}
\|u(T)-U(T)\|_{1} & \leq\left\|u(T)-u^{m}(T)\right\|_{1}+\left\|u^{m}(T)-U(T)\right\|_{1} \\
& =\mathcal{O}\left(\Delta \tau^{\alpha / 2}+\left|y^{m}\right|_{B V([0, T])} \sqrt{\Delta x}\right) \\
& =\mathcal{O}\left(\Delta \tau^{\alpha / 2}\right) .
\end{aligned}
$$

And by (5.6) and (5.8),

$$
\begin{aligned}
N & =\sum_{k=0}^{\tilde{L}(m)-1} \tilde{n}(k) \leq \sum_{k=0}^{\tilde{L}(m)-1}\left(\frac{\left|\Delta \tilde{y}_{k}^{m}\right|\left\|f^{\prime}\right\|_{L^{\infty}}}{C_{\mathrm{CFL}} \Delta x}+1\right) \\
& \leq \frac{\left\|f^{\prime}\right\|_{L^{\infty}}}{C_{\mathrm{CFL}} \Delta x}\left|y^{m}\right|_{B V([0, T])}+\tilde{L}(m) \\
& =\mathcal{O}\left(m^{\alpha} \max \left(\left|y^{m}\right|_{B V([0, T])}^{3}, 1\right)+\tilde{L}(m)\right) .
\end{aligned}
$$

In order to obtain 5.9 , it remains to verify that

$$
\tilde{L}(m)=\mathcal{O}\left(m^{\alpha} \max \left(\left|y^{m}\right|_{B V([0, T])}^{3}, 1\right)\right) .
$$

Assume condition (b) holds and that $m \geq \tilde{m}$. Since $\left\{\tilde{\tau}_{k}\right\}_{k=0}^{\tilde{L}(m)-1} \subset A^{ \pm}\left[z^{m}\right]$ and $\left\{\left|\Delta \tilde{y}_{k}^{m}\right|\right\}_{k=0}^{\tilde{L}(m)-2}$ is a monotonically increasing sequence, cf. 5.2 , it holds for any $\tilde{\tau}_{r} \in\left\{\tilde{\tau}_{k}\right\}_{k=0}^{\tilde{\tilde{L}}(m)-2} \cap\left[\tau_{\left\lceil m^{\alpha}\right\rceil}, T\right)$ that

$$
\left|\Delta \tilde{y}_{r}^{m}\right| \geq \max \left(M^{+}\left[z^{m}\right]\left(\tilde{\tau}_{r}\right),\left|M^{-}\left[z^{m}\right]\left(\tilde{\tau}_{r}\right)\right|\right) \geq \tilde{c} m^{-\alpha} .
$$

Moreover, the following function is well-defined for all $m \geq \tilde{m}$ :

$$
r(m):=\min \left\{k \in\left\{0,1, \ldots,\left\lceil m^{\alpha}\right\rceil\right\}|| \Delta \tilde{y}_{k}^{m} \mid>\tilde{c} m^{-\alpha}\right\} .
$$


It is clear that $r(m)=\mathcal{O}\left(m^{\alpha}\right)$ and

$$
\left|\tilde{y}^{m}\right|_{B V([0, T])}=\sum_{k=0}^{\tilde{L}(m)-1}\left|\Delta \tilde{y}_{k}^{m}\right| \geq \sum_{k=r(m)}^{\tilde{L}(m)-2} m^{-\alpha}=(\tilde{L}(m)-(r(m)+2)) m^{-\alpha} .
$$

We conclude that

$$
\tilde{L}(m)=\mathcal{O}\left(m^{\alpha} \max \left(\left|y^{m}\right|_{B V([0, T])}, 1\right)\right)=\mathcal{O}\left(m^{\alpha} \max \left(\left|y^{m}\right|_{B V([0, T])}^{3}, 1\right)\right),
$$

and thus condition (b) is stronger than condition (a) (condition (b) is included in the theorem as it might be easier to verify than condition (a)).

The computational cost of the numerical method is equal to the sum of $\Theta\left(m^{\beta}\right)$ for generating the piecewise linear interpolant $z^{m}$, and

$$
\Theta\left(N \times \frac{b_{m}-a_{m}}{\Delta x}\right)
$$

for solving $U$ over $[0, T] \times\left[a_{m}, b_{m}\right]$.

5.3. Efficiency gains and case study of Wiener paths. By comparing the computational cost versus accuracy results in Theorems 3.4 and 5.2 , we see that if the assumptions of both theorems hold,

and

$$
\limsup _{m \rightarrow \infty} \frac{m^{\beta}}{m^{2 \alpha}\left|z^{m}\right|_{B V([0, T])}^{5}}=0,
$$

$$
\limsup _{m \rightarrow \infty} \frac{\left|\overline{\mathrm{orm}}_{m}[z]\right|_{B V([0, T])}^{6}}{\left|z^{m}\right|_{B V([0, T])}^{5}}=0
$$

then it is guaranteed that the orm based numerical method will be asymptotically more efficient than the adaptive timestep method.

The next two lemmas verify that $\left|\overline{\operatorname{orm}}_{m}[z]\right|_{B V([0, T])}<\infty$, for all $m \geq 2$, and that assumption (b) in Theorem 5.2 holds for almost all sample paths of a standard Wiener process.

Lemma 5.3. Let $(\Omega, \mathcal{F}, \mathbb{P})$ denote a probability space on which the standard Wiener process $W:[0, \infty) \times \Omega \rightarrow \mathbb{R}$ with $W(0)=0, \mathbb{P}$-a.s. is defined. For every $\omega \in \Omega$, let $z:=W(\cdot, \omega)$ denote a sample path of the Wiener process. Then, for every $\alpha \in(0,1 / 2)$ and $\widehat{T}>0$

$$
z \in C_{0}^{0, \alpha}([0, \widehat{T}]), \quad \mathbb{P}-\text { a.s. }
$$

Furthermore, for a fixed $T>0$, let

$$
\widetilde{\Omega}=\left\{\omega \in \Omega \mid W(\cdot, \omega) \in C_{0}([0, T])\right\},
$$

and for any $m \geq 2$, let $\left\{\tau_{k}\right\}_{k=0}^{m} \subset[0, T]$ denote the uniform mesh with step size $\Delta \tau=T / m$. We define

$$
z^{m}:= \begin{cases}\mathcal{I}[z]\left(\cdot ;\left\{\tau_{k}\right\}_{k=0}^{m}\right) & \text { if } \omega \in \widetilde{\Omega}, \\ 0 & \text { if } \omega \in \Omega \backslash \widetilde{\Omega},\end{cases}
$$

and, as a consequence of that,

$$
\overline{\operatorname{orm}}_{m}[z]:= \begin{cases}\overline{\operatorname{orm}}[z]\left(\cdot ;\left\{\tau_{k}\right\}_{k=0}^{m}\right) & \text { if } \omega \in \widetilde{\Omega}, \\ 0 & \text { if } \omega \in \Omega \backslash \widetilde{\Omega} .\end{cases}
$$

Then

$$
\limsup _{m \rightarrow \infty} \sup _{t \in[0, T]}\left|z-z^{m}\right|(t) \sqrt{\frac{m}{\log (m)}} \leq \sqrt{2 T}, \quad \mathbb{P} \text {-a.s. }
$$




$$
\limsup _{m \rightarrow \infty}\left|z^{m}\right|_{B V([0, T])} \sqrt{\frac{\log (m)}{m}} \geq \sqrt{\frac{T}{2}}, \quad \mathbb{P} \text {-a.s., }
$$

and for $\mathbb{P}$-almost all paths, there is a constant $C(\omega)>0$ such that

$$
\limsup _{m \rightarrow \infty}\left|\overline{\mathrm{orm}}_{m}[z]\right|_{B V([0, T])}<C(\omega) .
$$

Moreover,

$$
\mathbb{E}\left[\sup _{m \geq 2}\left|\overline{\operatorname{orm}}_{m}[z]\right|_{B V([0, T])}\right]<\infty, \quad \mathbb{E}\left[|\operatorname{orm}[z]|_{B V([0, T])}\right]<\infty .
$$

Proof. See 29] for a proof of (5.14).

Since $\mathbb{P}(\widetilde{\Omega})=1$, we restrict ourselves to $\omega \in \widetilde{\Omega}$ in what follows. By Lévy's global modulus of continuity [53, Theorem 9.25$] \mathrm{L}^{2}$,

$$
\limsup _{\delta \downarrow 0} \sup _{\substack{0 \leq s \leq t \leq T \\ t-s \leq \delta}} \frac{|z(t)-z(s)|}{\sqrt{2 \delta \log (1 / \delta)}}=T, \quad \mathbb{P} \text {-a.s., }
$$

and as

$$
\begin{aligned}
\sup _{t \in[0, T]}\left|z(t)-z^{m}(t)\right| & \leq \max _{\substack{k \in\{0,1, \ldots, m-1\} \\
\sup _{t \in\left[\tau_{k}, \tau_{k+1}\right]}}} \max \left(\left|z(t)-z\left(\tau_{k}\right)\right|,\left|z(t)-z\left(\tau_{k+1}\right)\right|\right) \\
& \leq \sup _{\substack{0 \leq s \leq t \leq T \\
t-s \leq T / m}}|z(t)-z(s)|,
\end{aligned}
$$

inequality 5.18 follows and so does

$$
\limsup _{m \rightarrow \infty}\left(\max _{k \in\{0,1, \ldots, m-1\}}\left|\Delta z_{k}^{m}\right| \sqrt{\frac{m}{\log (m)}}\right) \leq \sqrt{2 T}, \quad \mathbb{P}-\text { a.s. }
$$

We further recall from [25] that

$$
\limsup _{m \rightarrow \infty} \sum_{k=1}^{m-1}\left|\Delta z_{k}^{m}\right|^{2}=\lim _{m \rightarrow \infty} \sum_{k=1}^{m-1}\left|\Delta z_{k}^{m}\right|^{2}=T, \quad \mathbb{P} \text {-a.s. }
$$

Hence,

$$
\begin{aligned}
T & =\limsup _{m \rightarrow \infty} \sum_{k=1}^{m-1}\left|\Delta z_{k}^{m}\right|^{2} \\
\leq & \limsup _{m \rightarrow \infty}\left(\max _{\ell \in\{0,1, \ldots, m-1\}}\left|\Delta z_{\ell}^{m}\right| \sum_{k=1}^{m-1}\left|\Delta z_{k}^{m}\right|\right) \\
\leq & \left.\limsup _{m \rightarrow \infty}\left(\max _{k \in\{0,1, \ldots, m-1\}}\left|\Delta z_{k}^{m}\right| \sqrt{\frac{m}{\log (m)}}, \sqrt{2 T}\right)\right) \\
& \times \limsup _{m \rightarrow \infty}\left(\left|z^{m}\right|_{B V([0, T])} \sqrt{\frac{\log (m)}{m}}\right) \\
= & \sqrt{2 T} \limsup _{m \rightarrow \infty}\left(\left|z^{m}\right|_{B V([0, T])} \sqrt{\frac{\log (m)}{m}}\right), \quad \mathbb{P} \text {-a.s., }
\end{aligned}
$$

and $(5.19)$ follows.

Equations (5.20) and (5.21) are proved in Appendix B.

\footnotetext{
${ }^{2}$ Theorem 9.25 in $[53$ is formulated for standard Wiener processes over the time interval $[0,1]$. However, for any $T>0$, the transform $\widetilde{W}(t)=\sqrt{T} W(t / T)$ yields a standard Wiener process $\widetilde{W}:[0, T] \times \Omega \rightarrow \mathbb{R}$ and the result extends straightforwardly to the time interval $[0, T]$.
} 
The next lemma shows that assumption (b) in Theorem 5.2 holds for almost all sample paths of a standard Wiener process.

Lemma 5.4. For any $m \geq 2$, let $\left\{\tau_{k}\right\}_{k=0}^{m} \subset[0, T]$ denote the uniform mesh with with step size $\Delta \tau=T / m$. Let $z=W(\cdot, \omega) \in C_{0}([0, T])$ denote a path realization of the standard Wiener process with $\omega \in \widetilde{\Omega}$, cf. Lemma 5.3 and $(5.15)$, and let $z^{m}$ be defined by (5.16). Then, for any $\alpha \in(2 / 5,1]$, there exists an $\tilde{m}(\omega) \geq 2$ for almost all $\omega \in \widetilde{\Omega}$ such that

$$
\max \left(M^{+}\left[z^{m}\right]\left(\tau_{\left\lceil m^{\alpha}\right\rceil}\right),\left|M^{-}\left[z^{m}\right]\left(\tau_{\left\lceil m^{\alpha}\right\rceil}\right)\right|\right) \geq m^{-\alpha} \quad \forall m \geq \tilde{m}(\omega) .
$$

Proof. For $y \in \mathbb{R}$, let $\lfloor y\rfloor$ denote the largest $n \in \mathbb{Z}$ such that $y \geq n$ and let $r^{m}$ : $\mathbb{N} \rightarrow \mathbb{N}$ be defined by $r^{m}(k)=\left\lfloor m^{\alpha / 2}\right\rfloor k$. For any natural number $m \geq \hat{m}=\left\lceil 3^{2 / \alpha}\right\rceil$, we introduce the set

$$
D_{m}=\left\{\omega \in \tilde{\Omega}|| z\left(\tau_{r^{m}(k+1)}\right)-z\left(\tau_{r^{m}(k)}\right) \mid \leq \frac{2}{m^{\alpha}} \quad \text { for } \quad k=0,1, \ldots,\left\lfloor m^{\alpha / 2}\right\rfloor-1\right\} .
$$

We claim that any $\omega \in \widetilde{\Omega}$ for which $(5.22)$ does not hold is contained in the set

$$
\bigcap_{m \geq \hat{m}} \bigcup_{\ell \geq m} D_{\ell}
$$

To verify this, observe that if $\omega \notin \bigcap_{m \geq \hat{m}} \bigcup_{\ell>m} D_{\ell}$, then there exists an $\tilde{m}(\omega)$ such that $\omega \notin \bigcup_{\ell \geq \tilde{m}} D_{\ell}$. Since $\omega \notin D_{m}$ for every $m \geq \tilde{m}$, there exists a $k_{m} \in$ $\left\{0,1, \ldots,\left\lfloor m^{\alpha / 2}\right\rfloor-1\right\}$ such that $\left|z\left(\tau_{r^{m}\left(k_{m}+1\right)}\right)-z\left(\tau_{r^{m}\left(k_{m}\right)}\right)\right| \geq \frac{2}{m^{\alpha}}$. This implies that $\max \left(\left|z\left(\tau_{r^{m}\left(k_{m}+1\right)}\right)\right|,\left|z\left(\tau_{r^{m}\left(k_{m}\right)}\right)\right|\right)>m^{-\alpha}$, and, since $r^{m}\left(k_{m}+1\right) \leq\left\lceil m^{\alpha}\right\rceil$ by construction, we conclude that

$$
\max \left(M^{+}\left[z^{m}\right]\left(\tau_{\left\lceil m^{\alpha}\right\rceil}\right),\left|M^{-}\left[z^{m}\right]\left(\tau_{\left\lceil m^{\alpha}\right\rceil}\right)\right|\right) \geq m^{-\alpha}, \quad \forall m \geq \tilde{m} .
$$

We will show that $(5.23)$ is a zero-measure set. Since the increments $\left\{z\left(\tau_{r^{m}(k+1)}\right)-\right.$ $\left.z\left(\tau_{r^{m}(k)}\right)\right\}_{k}$ are independent $N\left(0, \tau_{\left\lfloor m^{\alpha / 2}\right\rfloor}\right)$ distributed random variables,

$$
\mathbb{P}\left(D_{m}\right) \leq\left(\mathbb{P}\left(\left|z\left(\tau_{r^{m}(1)}\right)-z(0)\right| \leq \frac{2}{m^{\alpha}}\right)\right)^{\left\lfloor m^{\alpha / 2}\right\rfloor}
$$

and since $\tau_{\left\lfloor r^{m}(1)\right\rfloor}=\Theta\left(m^{\alpha / 2-1}\right)$, there exists a $C>0$ independent of $m$ such that for all $m \geq \hat{m}$,

$$
\begin{aligned}
\mathbb{P}\left(\left|z\left(\tau_{r^{m}(1)}\right)-z(0)\right| \leq \frac{2}{m^{\alpha}}\right) & =\frac{1}{\sqrt{2 \pi \tau_{r^{m}(1)}}} \int_{-2 m^{-\alpha}}^{2 m^{-\alpha}} \exp \left(\frac{-x^{2}}{2 \tau_{r^{m}(1)}}\right) \mathrm{d} x \\
& =\frac{1}{\sqrt{2 \pi}} \int_{\frac{-2 m-\alpha}{\sqrt{\tau^{m}(1)}}}^{\frac{2 m^{-\alpha}}{\sqrt{\tau^{m}(1)}}} e^{-y^{2} / 2} \mathrm{~d} y \\
& \leq C m^{1 / 2-5 \alpha / 4} .
\end{aligned}
$$

Since $1 / 2-5 \alpha / 4<0$, there exists a $\check{m} \geq \hat{m}$, such that

$$
\mathbb{P}\left(D_{m}\right) \leq m^{-2} \text { for all } m \geq \check{m},
$$

and

$$
\mathbb{P}\left(\bigcap_{m \geq \hat{m} \ell \geq m} \bigcup_{\ell} D_{\ell}\right) \leq \liminf _{m \rightarrow \infty} \mathbb{P}\left(\bigcup_{\ell \geq m} D_{\ell}\right) \leq \liminf _{m \rightarrow \infty} \sum_{\ell=m}^{\infty} \mathbb{P}\left(D_{\ell}\right) \leq \lim _{m \rightarrow \infty} \frac{1}{m}=0
$$

\footnotetext{
${ }^{3}$ By a slight modification of the proof, one may show that for almost all $\omega \in \widetilde{\Omega}$, inequality 5.22
} holds for any $\alpha \in(1 / 4,1]$. 
Equations (5.14) and 5.15) ensure that

$$
\mathbb{P}\left(\widetilde{\Omega} \backslash \bigcap_{m \geq \hat{m} \ell \geq m} \bigcup_{\ell} D_{\ell}\right)=1
$$

and the proof follows.

By the "Hölder continuity" (5.18), equations (5.19) and (5.20), and the fact that $\beta=1$ for sampling a path of a standard Wiener processes, we conclude that under the shared assumptions on $u_{0}$ in Theorems 3.4 and 5.2 and if $f \in C^{2}(\mathbb{R})$ is strictly convex, then the computational cost of achieving

$$
\|u(T)-U(T)\|_{L^{1}}=\mathcal{O}\left(\left(\frac{\log (m)}{m}\right)^{1 / 4}\right),
$$

for a sample path $z:[0, T] \rightarrow \mathbb{R}$ of the standard Wiener process admits for some $c_{1}(\omega)>0$ the following lower bound for the adaptive time stepping method (cf. Theorem 3.4.

$$
\operatorname{Cost}(U(T)) \geq c_{1}\left(\frac{m}{\log (m)}\right)^{7 / 2} \quad \mathbb{P} \text {-a.s. }
$$

and, for some $c_{2}(\omega)>0$, the following upper bound for the orm based method (cf. Theorem 5.2):

$$
\operatorname{Cost}(U(T)) \leq c_{2} m \quad \mathbb{P} \text {-a.s. }
$$

Remark 5.1. In many cases (e.g. Brownian paths), $|\operatorname{orm}[z]|_{B V([0, T])}<|z|_{B V([0, T])}$. That is however not always the case. The second example in Figure 9 considers the path $z(t)=\mathbf{1}_{t>0} t \sin (\pi /(2 t))$, which is a member of $C_{0}^{0,1 / 2}([0, T])$ for which $|\operatorname{orm}[z]|_{B V([0, T])}=|z|_{B V([0, T])}=\infty$. This shows that even if $f \in C^{2}(\mathbb{R})$ is strictly convex, the orm based method will not always solve 1.5 more efficiently than the adaptive timestepping method.

\subsection{Numerical tests.}

Example 5.1. To investigate if properties similar to those in Lemma 5.3 hold for more general Hölder continuous paths (beyond Wiener paths), we approximate $\mathbb{E}\left[\left|z^{m}\right|_{B V([0,1])}\right]$ and $\mathbb{E}\left[\left|\overline{\operatorname{orm}}_{m}[z]\right|_{B V([0,1])}\right]$ for fBMs with respective Hurst indices $\alpha=1 / 8,1 / 4,1 / 2$ and $3 / 4$ on uniform meshes of $[0,1]$ with step sizes $\Delta \tau=1 / \mathrm{m}$ for $m=2^{5}, 2^{6}, \ldots, 2^{16}$. The expectations are approximated by the Monte Carlo method using $10^{6}$ sample realizations of $\left|z^{m}\right|_{B V([0,1])}$ and $\left|\overline{\operatorname{orm}}_{m}[z]\right|_{B V([0,1])}$. Our Monte Carlo estimates of the expectations are presented in Figure 11. We observe that while $\mathbb{E}\left[\left|z^{m}\right|_{B V([0,1])}\right]=\Theta\left(m^{1-\alpha}\right), \mathbb{E}\left[\left|\overline{\operatorname{orm}}_{m}[z]\right|_{B V([0,1])}\right]$ seems to stay bounded as $m$ increases for all of the considered fBMs. In the bottom row of Figure 11, we have computed the "BV increment ratio"

$$
G(k):=\frac{\mathbb{E}\left[\left|\overline{\mathrm{orm}}_{2^{k+1}}[z]\right|_{B V([0,1])}-\left|\overline{\mathrm{orm}}_{2^{k}}[z]\right|_{B V([0,1])}\right]}{\mathbb{E}\left[\left|\overline{\mathrm{orm}}_{2^{6}}[z]\right|_{B V([0,1])}-\left|\overline{\mathrm{orm}}_{2^{5}}[z]\right|_{B V([0,1])}\right]},
$$

for $k \geq 5$. The increment ratio seems to be geometric of the form $G(k)=\mathcal{O}\left(\rho_{\alpha}^{k-5}\right)$ with $\rho_{\alpha} \approx 2^{-\alpha}<1$. We interpret this as numerical support for

$$
\mathbb{E}\left[\lim _{k \rightarrow \infty}\left|\overline{\mathrm{orm}}_{2^{k}}[z]\right|_{B V([0,1])}\right]<\infty, \quad \forall \alpha \in\{1 / 8,1 / 4,1 / 2,3 / 4\},
$$



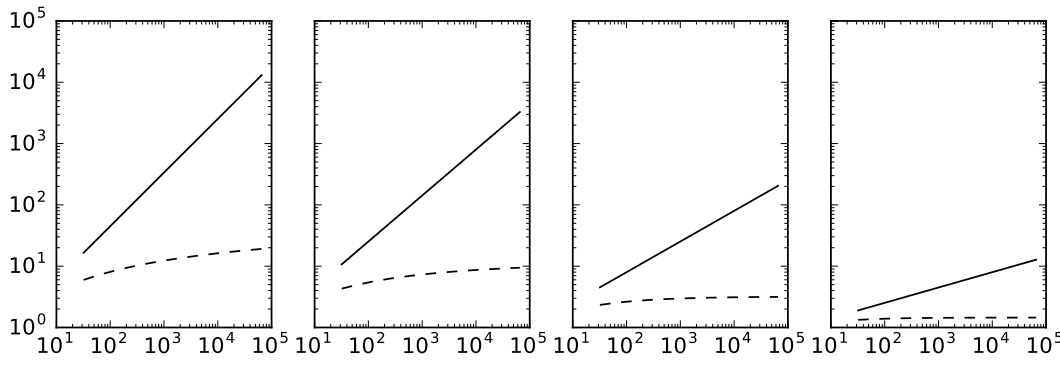

$m$

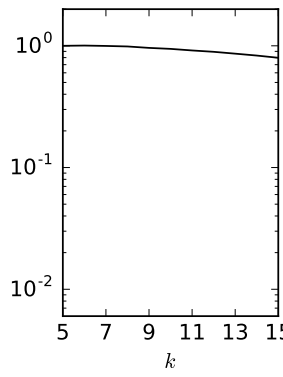

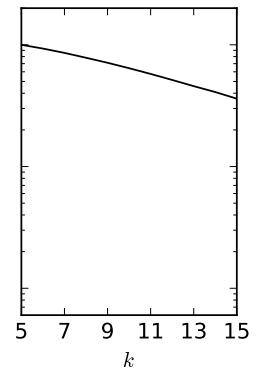

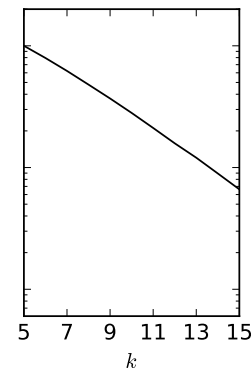

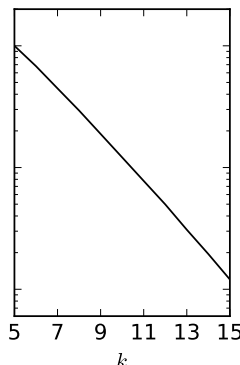

Figure 11. Top row: Example 5.1. Monte Carlo computations of $\mathbb{E}\left[\left|z^{m}\right|_{B V([0,1])}\right]$ (solid line) and $\mathbb{E}\left[\left|\overline{\operatorname{orm}}_{m}[z]\right|_{B V([0,1])}\right]$ (dashed line), Hurst indices $\alpha=0.125$ (left), $\alpha=0.25$ (second left), $\alpha=0.5$ (third left), and $\alpha=0.75$ (right). Bottom row: plots of the BV increment ratio $G(k)$, cf. (5.24), corresponding to the respective top row test cases.

since if $G(k)<1$,

$$
\begin{aligned}
& \mathbb{E}\left[\lim _{k \rightarrow \infty}\left|\overline{\mathrm{orm}}_{2^{k}}[z]\right|_{B V([0,1])}\right] \\
& =\mathbb{E}\left[\left|\overline{\mathrm{orm}}_{2^{5}}[z]\right|_{B V([0,1])}\right]+\sum_{k=5}^{\infty} \mathbb{E}\left[\left|\overline{\mathrm{orm}}_{2^{k+1}}[z]\right|_{B V([0,1])}-\left|\overline{\mathrm{orm}}_{2^{k}}[z]\right|_{B V([0,1])}\right] \\
& \leq C \sum_{k=0}^{\infty} \rho_{\alpha}^{k}<\infty
\end{aligned}
$$

Example 5.2. We consider problem 3.19 over the time interval $[0,1]$ with periodic boundary conditions, initial data

$$
u_{0}(x)=\operatorname{sign}(x-1 / 2) \mathbf{1}_{1 / 6<5 / 6} \quad x \in[0,1],
$$

strictly convex flux $f(u)=u / 2+u^{2} / 4$ and $z=W(\cdot, \omega)$, where $W:[0,1] \times \widetilde{\Omega} \rightarrow \mathbb{R}$ denotes the standard Wiener process and $\widetilde{\Omega}$ is defined in (5.15). In Figure 12 we compare the performance of the adaptive timestep method and the orm based method (cf. Section 5), both using the Lax-Friedrichs scheme. The driving path $z$ is piecewise linearly interpolated on two uniform mesh resolutions, $m=2^{8}$ and $m=2^{10}$, and the computational cost of the respective algorithms are equilibrated through

$$
\Delta x_{\mathrm{orm}}=\frac{\Delta x_{\text {adaptive }}}{\left\lceil\sqrt{\frac{\left|z^{m}\right|_{B V([0, T])}}{\left|\operatorname{orm}\left[z^{m}\right]\right|_{B V([0, T])}}}\right\rceil},
$$


(equilibrating either lower or upper bound costs in both of Theorems 3.4 and 4.7). The approximate reference solution is computed by the orm based method using the Lax-Friedrichs scheme with piecewise linear interpolation of $z$ on a uniform mesh with much higher resolution $\left(m=2^{14}\right)$. We observe that at comparable computational budget, the orm based method approximates the reference solution with better accuracy and produces less artificial diffusion than the adaptive timestep method.
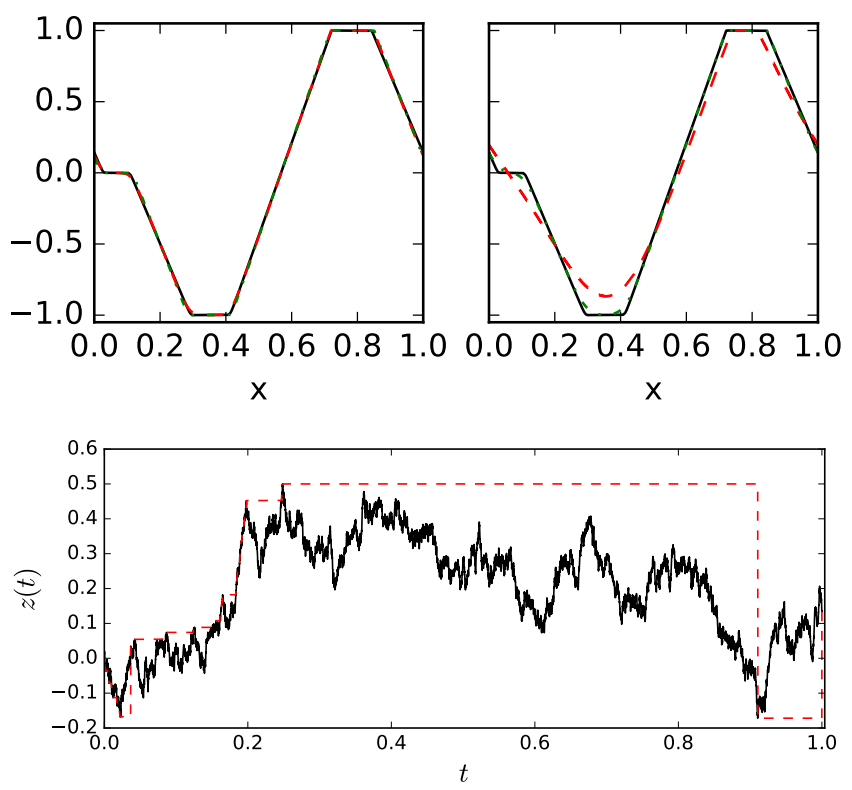

Figure 12. Top row: Final time solutions for Example 5.2 computed with the orm based method (left figure) and adaptive timestep method (right figure). The black solid line is the approximate reference solution $u(1)$, while the red dashed and the green dash-dotted lines are the numerical solutions at the driving path resolutions $m=2^{8}$ and $m=2^{10}$, respectively. Bottom: The driving path (black solid line) and the corresponding orm (red dashed line).

\section{Conclusion}

In this work we have developed fully discrete and thus computable numerical methods for solving conservation laws with driving paths of low regularity. For strictly convex flux functions, we have identified a class of "oscillatory cancellations" that can be removed from the driving path to produce numerical methods for computing final time entropy solutions with improved efficiency. If the driving path is realization of a Wiener process, for instance, the asymptotic efficiency gain can be of orders of magnitude. An in-depth study of the driving path cancellation property is found in the theoretical companion article [46].

An interesting follow up study, which we leave for future work, is to investigate whether the methodology involving "oscillatory cancellations" may lead to efficient methods for approximating not only the solution at final time $t=T$, but also its time evolution on $[0, T]$. 
There is a link between the theory developed in the recent preprint 34 and our work here and in [46. In [34] the authors use "oscillatory cancellations" (with different terminology) to derive new qualitative properties for convex HamiltonJacobi equations with low-regularity paths (in multiple dimensions). Although several results are similar, the proofs are very different from ours.

\section{Appendix A. Regularity of SOlutions}

Lemma A.1. For a given set of points

$$
0=\tau_{0}<\tau_{1}<\ldots<\tau_{m}=T, \quad m \geq 2,
$$

let $u^{m} \in C_{0}\left([0, T] ; L^{1}(\mathbb{R})\right)$ denote the càdlàg version of the entropy solution of (2.1) with initial data $u_{0} \in\left(L^{1} \cap B V\right)(\mathbb{R})$, driving path $z^{m} \in I_{0}\left([0, T] ;\left\{\tau_{j}\right\}_{j=0}^{m}\right)$ and strictly convex $f \in C^{2}(\mathbb{R})$. For any $x \in \mathbb{R}$ and $t \in[0, T]$, let

$$
u^{m}(t, x+):=\lim _{\delta \downarrow 0} u^{m}(t, x+\delta)(=u(t, x)) \quad \text { and } \quad u^{m}(t, x-):=\lim _{\delta \downarrow 0} u^{m}(t, x-\delta),
$$

Then, using the convention $0^{-1}=\infty$, it holds for all $x<y$ and $t \in[0, T]$ that

$$
-\frac{1}{M^{+}\left[z^{m}\right](t)-z^{m}(t)} \leq \frac{f^{\prime}\left(u^{m}(t, y \pm)\right)-f^{\prime}\left(u^{m}(t, x \pm)\right)}{y-x} \leq \frac{1}{z^{m}(t)-M^{-}\left[z^{m}\right](t)},
$$

where the functions $M^{-}\left[z^{m}\right]$ and $M^{+}\left[z^{m}\right]$ are defined in 4.11.

To prove Theorem 4.2 we will need the following intermediate result, which is an adaptation of [46, Lemma 3.3].

Lemma A.2. For a given set of points

$$
0=\tau_{0}<\tau_{1}<\ldots<\tau_{m}=T, \quad m \geq 2,
$$

let $u^{m} \in C_{0}\left([0, T] ; L^{1}(\mathbb{R})\right)$ denote the càdlàg version of the entropy solution of 2.1 with initial data $u_{0} \in\left(L^{1} \cap B V\right)(\mathbb{R})$, driving noise $z^{m} \in I_{0}\left([0, T] ;\left\{\tau_{j}\right\}_{j=0}^{m}\right)$ and strictly convex $f \in C^{2}(\mathbb{R})$. Assume that A.1 holds at some time $\tau_{k} \in\left\{\tau_{j}\right\}_{j=0}^{m-1}$. Then, for all $x<y$ and $s \in\left[\tau_{k}, \tau_{k+1}\right]$,

$$
-\frac{1}{M^{+}\left[z^{m}\right](s)-z^{m}(s)} \leq \frac{f^{\prime}\left(u^{m}(s, y \pm)\right)-f^{\prime}(u(s, x \pm))}{y-x} \leq \frac{1}{z^{m}(s)-M^{-}\left[z^{m}\right](s)} \text {. }
$$

Proof. For some $s \in\left(\tau_{k}, \tau_{k+1}\right]$, let $\zeta_{ \pm}$be the maximal/minimal backward generalized characteristic emanating from $(s, y)$ and $\xi_{ \pm}$be the maximal/minimal backward generalized characteristic emanating from $(s, x)$, cf. [18, $\S \mathrm{X}]$. The solution representation

$$
u^{m}(s)=\overline{\mathcal{S}}\left(z^{m}(s)-z^{m}\left(\tau_{k}\right)\right) u^{m}\left(\tau_{k}\right)=\mathcal{S}^{\dot{z}_{k}^{m}}\left(s-\tau_{k}\right) u^{m}\left(\tau_{k}\right),
$$

cf. (3.2) and (4.4), and [18, Theorem 11.1.1] implies that the generalized characteristics satisfies the following equations

$$
\begin{aligned}
& x=\xi_{ \pm}\left(\tau_{k}\right)+\left(s-\tau_{k}\right) \dot{z}_{k}^{m} f^{\prime}\left(u^{m}(s, x \pm)\right)=\xi_{ \pm}\left(\tau_{k}\right)+\left(z^{m}(s)-z^{m}\left(\tau_{k}\right)\right) f^{\prime}\left(u^{m}(s, x \pm)\right), \\
& y=\zeta_{ \pm}\left(\tau_{k}\right)+\left(s-\tau_{k}\right) \dot{z}_{k}^{m} f^{\prime}\left(u^{m}(s, y \pm)\right)=\zeta_{ \pm}\left(\tau_{k}\right)+\left(z^{m}(s)-z^{m}\left(\tau_{k}\right)\right) f^{\prime}\left(u^{m}(s, y \pm)\right),
\end{aligned}
$$

where $\xi_{ \pm}\left(\tau_{k}\right) \leq \zeta_{ \pm}\left(\tau_{k}\right)$ and each equation holds using either the limit sign + or consistently in all terms with the appendage \pm (i.e., $x+$ and $\xi_{+}\left(\tau_{k}\right)$ etc.); and we recall that $\dot{z}_{k}^{m}=\dot{z}^{m}\left(\tau_{k}+\right)$. We will treat the cases $\dot{z}_{k}^{m}>0$ and $\dot{z}_{k}^{m}<0$ separately (the case $\dot{z}_{k}^{m}=0$ is trivial since then $u^{m}(t)=\mathcal{S}^{0}\left(t-\tau_{k}\right) u^{m}\left(\tau_{k}\right)=u^{m}\left(\tau_{k}\right)$ for all $\left.t \in\left[\tau_{k}, \tau_{k+1}\right]\right)$. 
Assume $\dot{z}_{k}^{m}>0$. By [18, Theorem 11.1.3],

$$
\begin{aligned}
& u^{m}\left(\tau_{k}, \xi_{ \pm}\left(\tau_{k}\right)-\right) \leq u^{m}(s, x \pm) \leq u^{m}\left(\tau_{k}, \xi_{ \pm}\left(\tau_{k}\right)+\right), \\
& u^{m}\left(\tau_{k}, \zeta_{ \pm}\left(\tau_{k}\right)-\right) \leq u^{m}(s, y \pm) \leq u^{m}\left(\tau_{k}, \zeta_{ \pm}\left(\tau_{k}\right)+\right) .
\end{aligned}
$$

Under the assumption that $f^{\prime}\left(u^{m}(s, y \pm)\right)-f^{\prime}\left(u^{m}(s, x \pm)\right) \neq 0$ (otherwise A.2 holds trivially), we have

(A.4)

$$
\frac{f^{\prime}\left(u^{m}(s, y \pm)\right)-f^{\prime}(u(s, x \pm))}{y-x}=\frac{1}{\frac{\zeta_{ \pm}\left(\tau_{k}\right)-\xi_{ \pm}\left(\tau_{k}\right)}{f^{\prime}\left(u^{m}(s, y \pm)\right)-f^{\prime}(u(s, x \pm))}+\left(z^{m}(s)-z^{m}\left(\tau_{k}\right)\right)} .
$$

If $f^{\prime}\left(u^{m}(s, y \pm)\right)-f^{\prime}\left(u^{m}(s, x \pm)\right)<0$, then the upper bound of A.2 holds trivially, and inequalities A.3 and $f^{\prime}>0$ imply that

$$
0>f^{\prime}\left(u^{m}(s, y \pm)\right)-f^{\prime}\left(u^{m}(s, x \pm)\right) \geq f^{\prime}\left(u^{m}\left(\tau_{k}, \zeta_{ \pm}\left(\tau_{k}\right)-\right)\right)-f^{\prime}\left(u^{m}\left(s, \xi_{ \pm}\left(\tau_{k}\right)+\right)\right) .
$$

Hence, either $\zeta_{ \pm}\left(\tau_{k}\right)=\xi_{ \pm}\left(\tau_{k}\right)$ or $\zeta_{ \pm}\left(\tau_{k}\right)>\xi_{ \pm}\left(\tau_{k}\right)$. In the former case, the lower bound of A.2 holds since

$$
\frac{\zeta_{ \pm}\left(\tau_{k}\right)-\xi_{ \pm}\left(\tau_{k}\right)}{f^{\prime}\left(u^{m}(s, y \pm)\right)-f^{\prime}(u(s, x \pm))}+\left(z^{m}(s)-z^{m}\left(\tau_{k}\right)\right)=\left(z^{m}(s)-z^{m}\left(\tau_{k}\right)\right)>0
$$

(as we assume $\dot{z}_{k}^{m}>0$ ). In the latter case, since $u^{m}\left(\tau_{k}\right)$ is càdlàg, there exists a sequence $\left\{\xi_{ \pm, j}\left(\tau_{k}\right)\right\}_{j \in \mathbb{N}} \subset\left[\xi_{ \pm}\left(\tau_{k}\right), \zeta_{ \pm}\left(\tau_{k}\right)\right)$ such that $\xi_{ \pm, j}\left(\tau_{k}\right) \downarrow \xi_{ \pm}\left(\tau_{k}\right)$,

$$
f^{\prime}\left(u^{m}\left(\tau_{k}, \xi_{ \pm, j}\left(\tau_{k}\right)-\right)>f^{\prime}\left(u^{m}\left(\tau_{k}, \zeta_{ \pm}\left(\tau_{k}\right)-\right) \quad \forall j \in \mathbb{N}\right.\right.
$$

and

$$
\lim _{j \rightarrow \infty} f^{\prime}\left(u^{m}\left(\tau_{k}, \xi_{ \pm, j}\left(\tau_{k}\right)-\right)=f^{\prime}\left(u^{m}\left(\tau_{k}, \xi_{ \pm}\left(\tau_{k}\right)+\right)\right.\right.
$$

By A.1,

$$
\begin{aligned}
\frac{\zeta_{ \pm}\left(\tau_{k}\right)-\xi_{ \pm}\left(\tau_{k}\right)}{f^{\prime}\left(u^{m}(s, y \pm)\right)-f^{\prime}(u(s, x \pm))} & \leq \lim _{j \rightarrow \infty} \frac{\zeta_{ \pm}\left(\tau_{k}\right)-\xi_{ \pm, j}\left(\tau_{k}\right)}{f^{\prime}\left(u^{m}\left(\tau_{k}, \zeta_{ \pm}\left(\tau_{k}\right)-\right)\right)-f^{\prime}\left(u\left(\tau_{k}, \xi_{ \pm, j}\left(\tau_{k}\right)-\right)\right)} \\
& \leq z^{m}\left(\tau_{k}\right)-M^{+}\left[z^{m}\right]\left(\tau_{k}\right),
\end{aligned}
$$

which in combination with A.4 shows that the lower bound of A.2 holds.

So far, we have verified the lemma in the following situations:

(i) $\dot{z}_{k}^{m}=0$,

(ii) $f^{\prime}\left(u^{m}(s, y \pm)\right)-f^{\prime}(u(s, x \pm))=0$,

(iii) $f^{\prime}\left(u^{m}(s, y \pm)\right)-f^{\prime}(u(s, x \pm))<0$ and $\dot{z}_{k}^{m}>0$.

To complete the proof it remains to verify the lemma for

(iv) $f^{\prime}\left(u^{m}(s, y \pm)\right)-f^{\prime}(u(s, x \pm))<0$ and $\dot{z}_{k}^{m}<0$,

(v) $f^{\prime}\left(u^{m}(s, y \pm)\right)-f^{\prime}(u(s, x \pm))>0$ and $\dot{z}_{k}^{m} \neq 0$.

These cases can be proved in a similar fashion as (iii). We refer to [46, Lemma 3.3] for further details.

Proof of Theorem 4.2. For an arbitrary $t \in[0, T]$, let us assume that $t \in\left[\tau_{k}, \tau_{k+1}\right]$ for some $0 \leq k \leq m-1$. For any $0 \leq j \leq m$, let $P_{j}$ be the statement: for all $-\infty<x<y<\infty$,

$$
\begin{aligned}
\left(P_{j}\right)-\frac{1}{M^{+}\left[z^{m}\right]\left(\tau_{j}\right)-z\left(\tau_{j}\right)} \leq & \\
\frac{f^{\prime}\left(u^{m}\left(\tau_{j}, y \pm\right)\right)-f^{\prime}\left(u^{m}\left(\tau_{j}, x \pm\right)\right)}{y-x} & \leq \frac{1}{z\left(\tau_{j}\right)-M^{-}\left[z^{m}\right]\left(\tau_{j}\right)} .
\end{aligned}
$$


The statement $P_{0}$,

$$
-\infty \leq \frac{f^{\prime}\left(u_{0}(y \pm)\right)-f^{\prime}\left(u_{0}(x \pm)\right)}{y-x} \leq \infty, \text { for all }-\infty<x<y<\infty,
$$

is obviously true. Furthermore, if $P_{j}$ is true, Lemma A.2 implies that also $P_{j+1}$ is true. By induction, we conclude that $P_{k}$ is true and, using Lemma A.2 once more, we conclude that A.1 holds for the considered $t \in\left[\tau_{k}, \tau_{k+1}\right]$.

\section{APPENDIX B. ORM, WIENER PATHS AND BOUNDED TOTAL VARIATION}

Before proceeding with the proof of equations $(5.20)$ and 5.21 of Lemma 5.3 . we recall a few useful properties on downcrossings for standard Wiener processes.

Theorem B.1. Let $a<m<b$ and consider a standard Wiener process $W$ : $[0, \infty) \times \Omega \rightarrow \mathbb{R}$ with $W(0)=m, \mathbb{P}$-a.s. Set $t^{*}=\min \{t \geq 0 \mid W(t) \in\{a, b\}\}$. Then

$$
\mathbb{P}\left(W\left(t^{*}\right)=a\right)=\frac{b-m}{b-a}, \quad \mathbb{P}\left(W\left(t^{*}\right)=b\right)=\frac{m-a}{b-a},
$$

and

$$
\mathbb{E}\left[t^{*}\right]=(m-a)(b-m) .
$$

For a proof of Theorem B.1, see [70, Theorem 2.49].

Definition B.1 (Downcrossing function). Let $m>a$ and consider a standard Wiener process $W:[0, \infty) \times \Omega \rightarrow \mathbb{R}$ with $W(0)=m$. Introduce the stopping times $\hat{\nu}_{0}=0$ and for $j \geq 1$,

$$
\check{\nu}_{j}=\inf \left\{t>\hat{\nu}_{j-1} ; W(t)=a\right\}, \quad \hat{\nu}_{j}=\inf \left\{t>\check{\nu}_{j} ; W(t)=m\right\} .
$$

The function $W_{\downarrow}^{(j)}(\cdot, \omega):\left[0, \check{\nu}_{j}-\hat{\nu}_{j-1}\right] \rightarrow \mathbb{R}$ defined by

$$
W_{\downarrow}^{(j)}(s)=W\left(\hat{\nu}_{j-1}(\omega)+s, \omega\right),
$$

thus represents the $j$ th downcrossing of $[a, m]$ for the Wiener path $W(\cdot, \omega)^{4}$ For $t>0$, we denote the number of downcrossings of $[a, m]$ completed before time $t$ by

$$
D(a, m, t):=\max \left\{j \in \mathbb{N} ; \check{\nu}_{j} \leq t\right\} .
$$

See [70, Section 6] for details on downcrossings for standard Wiener processes.

For $a<m<b$, a standard Wiener process $W:[0, \infty) \times \Omega \rightarrow \mathbb{R}$ with $W(0)=m$, $\mathbb{P}$-a.s. and the stopping time

$$
\mathfrak{t}^{b}:=\inf \{t \geq 0 ; W(t)=b\},
$$

it follows from Theorem B.1 and Definition B.1 that

$$
D\left(a, m, \mathfrak{t}^{b}\right) \sim \operatorname{Geo}\left(\frac{m-a}{b-a}\right) .
$$

Here, $\operatorname{Geo}(p)$ denotes the geometric distribution with parameter $p \in(0,1]$, which for $X \sim \operatorname{Geo}(p)$ has probability mass function

$$
\mathbb{P}(X=k)=p(1-p)^{k}, \quad k=0,1, \ldots,
$$

and

$$
\mathbb{E}[X]=(1-p) / p, \quad \mathbb{E}\left[X^{2}\right]=(1-p)(2-p) / p^{2},
$$

cf. [27].

\footnotetext{
${ }^{4}$ The time $\hat{\nu}_{0}$ is the first time $W(\cdot, \omega)$ equals $m$ and $\check{\nu}_{1}$ is the first time after $\hat{\nu}_{0}$ that $W(\cdot, \omega)$ equals $a$. Thus $W\left(\left[\hat{\nu}_{0}, \check{\nu}_{1}\right], \omega\right)$ represents the first downcrossing of $[a, m]$. The time $\hat{\nu}_{1}$ is the first time after $\check{\nu}_{1}$ that $W(\cdot, \omega)$ equals $m$ and $\check{\nu}_{2}$ is the first time after $\hat{\nu}_{1}$ that $W(\cdot, \omega)$ equals $a$. Thus $W\left(\left[\hat{\nu}_{1}, \check{\nu}_{2}\right], \omega\right)$ represents the second downcrossing of $[a, m] \ldots$
} 
Proof of equations (5.20) and (5.21). Recalling (5.15), it suffices to consider Wiener paths $z=W(\cdot, \omega)$ for $\omega \in \widetilde{\Omega}$. Moreover, since

$$
|\operatorname{orm}[z]|_{B V([0, T])} \leq|\max (\operatorname{orm}[z], 0)|_{B V([0, T])}+|\min (\operatorname{orm}[z], 0)|_{B V([0, T])},
$$

and, by symmetry, since the sample path $z$ has the same probability as the sample path $-z$ and $\operatorname{orm}[z]=-\operatorname{orm}[-z]$, cf. 4.24,

$$
\mathbb{E}\left[|\max (\operatorname{orm}[z], 0)|_{B V([0, T])}\right]=\mathbb{E}\left[|\min (\operatorname{orm}[z], 0)|_{B V([0, T])}\right],
$$

it suffices to verify that

$$
\mathbb{E}\left[\left|y^{\dagger}\right|_{B V([0, T])}\right]<\infty
$$

where $y^{\dagger}:=\mathbf{1}_{\omega \in \widetilde{\Omega}} \max (\operatorname{orm}[z], 0)$. Introduce the stopping times

$$
\check{\xi}_{j}=\inf \left\{t>0 ; z(t)=2^{-j}\right\}, \quad j \in \mathbb{Z}
$$

and note that $D\left(0,2^{-j}, \check{\xi}_{j-1}-\check{\xi}_{j}\right)$ equals the number of $z$-downcrossings of $\left[0,2^{-j}\right]$ completed in the time interval $\left[\check{\xi}_{j}, \check{\xi}_{j-1}\right]$ (i.e., between the first time $z$ equals $2^{-j}$ and the first time it equals $\left.2^{-j+1}\right)$. By (B.1), it follows that

$$
D\left(0,2^{-j}, \check{\xi}_{j-1}-\check{\xi}_{j}\right) \sim \operatorname{Geo}\left(\frac{1}{2}\right) .
$$

Suppose that

$$
\bar{t} \in\left\{t \in[0, T) \mid y^{\dagger}(t-)>y^{\dagger}(t)\right\} .
$$

Then, since $M^{+}[z]$ is a monotonically increasing function, $y^{\dagger}(\bar{t})=0$ (assuming otherwise,

$$
y^{\dagger}(\bar{t})>0 \Longrightarrow y^{\dagger}(\bar{t})=M^{+}[z](\bar{t})
$$

leads to a contradiction since $\left.y^{\dagger}(\bar{t})<y^{\dagger}(\bar{t}-) \leq M^{+}[z](\bar{t})\right)$. It therefore must hold that

and

$$
\bar{A}^{-}[z](\bar{t})>\bar{A}^{+}[z](\bar{t}), \quad \bar{A}^{-}[z](\bar{t}-)<\bar{A}^{+}[z](\bar{t}-)
$$

$$
y^{\dagger}(\bar{t}-)-y^{\dagger}(\bar{t})=M^{+}[z](\bar{t})
$$

cf. Definition 4.5. Hence,

$$
y^{\dagger}(\bar{t}-)-y^{\dagger}(\bar{t})<2^{\left\lfloor\log _{2}\left(M^{+}[z](\bar{t})\right)\right\rfloor+1},
$$

and

$$
\operatorname{orm}[z](\bar{t}-)=z\left(\bar{A}^{+}[z](\bar{t}-)\right)>0 \quad \text { and } \quad \operatorname{orm}[z](\bar{t})=z\left(\bar{A}^{-}[z](\bar{t})\right) \leq 0 .
$$

Consequently, any jump-discontinuity of the form $\mathrm{B} .3)$ with $j=\left\lfloor\log _{2}\left(M^{+}[z](t)\right)\right\rfloor$ must be preceded by a $z$-downcrossing of $\left[0,2^{-j}\right]$ within the time interval $\left[\check{\xi}_{j}, \check{\xi}_{j-1}\right]$ and $\bar{t} \in\left[\check{\xi}_{j}, \check{\xi}_{j-1}\right]$. (For $t<\check{\xi}_{j}$, jump-discontinuities in $y^{\dagger}$ of magnitude greater or equal to $2^{-j}$ cannot happen, and at later times, $t>\check{\xi}_{j-1}$, all jump-discontinuities of $y^{\dagger}$ will have magnitude greater than $2^{-j+1}>M^{+}[z](t)$.) Consequently, the jumpdiscontinuity $\bar{t}$ may be associated uniquely to the latest $z$-downcrossing of $\left[0,2^{-j}\right]$ in the time interval $\left[\check{\xi}_{j}, \check{\xi}_{j-1}\right]$ that precedes $t=\bar{t}$, and the mapping constituting this association, from the set (B.3) to the set

$$
\bigcup_{k \in \mathbb{Z}}\left\{z \text {-downcrossings of }\left[0,2^{-k}\right] \text { in time interval }\left[\check{\xi}_{k}, \check{\xi}_{k-1}\right]\right\}
$$

is thus injective.

Suppose next that

$$
\bar{t} \in\left\{t \in[0, T) \mid y^{\dagger}(t-)<y^{\dagger}(t)\right\}
$$


Then, since $z \in C_{0}([0, T]), M^{+}[z]$ is a continuous monotonically increasing function and $y^{\dagger}(\bar{t}-)=0$,

$$
\bar{A}^{+}[z](\bar{t}-)<\bar{A}^{-}[z](\bar{t}-), \quad \bar{A}^{+}[z](\bar{t})>\bar{A}^{-}[z](\bar{t})
$$

and

$$
y^{\dagger}(\bar{t})-y^{\dagger}(\bar{t}-)=M^{+}[z](\bar{t})
$$

Hence,

$$
\left|y^{\dagger}(\bar{t}-)-y^{\dagger}(\bar{t})\right|<2^{\left\lfloor\log _{2}\left(M^{+}[z](\bar{t})\right)\right\rfloor+1}
$$

and

$$
\operatorname{orm}[z](\bar{t}-)=z\left(\bar{A}^{-}[z](\bar{t}-)\right) \leq 0, \quad \operatorname{orm}[z](\bar{t})=z\left(\bar{A}^{+}[z](\bar{t})\right)>0 .
$$

By the continuity of $M^{+}[z]$,

$$
M^{+}[z](\bar{t}-)=z\left(\bar{A}^{+}[z](\bar{t}-)\right)=M^{+}[z](\bar{t}) .
$$

In other words, the time $s=\bar{A}^{+}[z](\bar{t}-)<\bar{A}^{-}[z](\bar{t}-)$ satisfies $z(s)=M^{+}[z](\bar{t})$. By the same reasoning as above, this implies that any jump-discontinuity of the form B.5 with $j=\left\lfloor\log _{2}\left(M^{+}[z](\bar{t})\right)\right\rfloor$ is preceded by a $z$-downcrossing of $\left[0,2^{-j}\right]$ in the time interval $\left[\check{\xi}_{j}, \check{\xi}_{j-1}\right]$, and, in fact, $\bar{t} \in\left[\check{\xi}_{j}, \check{\xi}_{j-1}\right]$. Consequently, the jumpdiscontinuity $\bar{t}$ may be associated uniquely to the latest $z$-downcrossing of $\left[0,2^{-j}\right]$ in the time interval $\left[\check{\xi}_{j}, \check{\xi}_{j-1}\right]$ that precedes $t=\bar{t}$, and the mapping constituting this association, from the set $(B .5)$ to the set $(B .4)$ is thus injective.

For any $j \in \mathbb{Z}$, let

$$
\mathfrak{B}_{j}^{-}:=\left\{t \in[0, T) \mid y^{\dagger}(t-)-y^{\dagger}(t) \in\left[2^{-j}, 2^{-j+1}\right)\right\}
$$

and

$$
\mathfrak{B}_{j}^{+}:=\left\{t \in[0, T) \mid y^{\dagger}(t)-y^{\dagger}(t-) \in\left[2^{-j}, 2^{-j+1}\right)\right\} .
$$

It then follows that for any $j \in \mathbb{Z}$,

$$
\sum_{\bar{t} \in \mathfrak{B}_{j}^{-}}\left|y^{\dagger}(\bar{t}-)-y^{\dagger}(\bar{t})\right| \leq 2^{-j+1} D\left(0,2^{-j}, \check{\xi}_{j-1}-\check{\xi}_{j}\right) \mathbf{1}_{\check{\xi}_{j}<T}
$$

and

$$
\sum_{\bar{t} \in \mathfrak{B}_{j}^{+}}\left|y^{\dagger}(\bar{t}-)-y^{\dagger}(\bar{t})\right| \leq 2^{-j+1} D\left(0,2^{-j}, \check{\xi}_{j-1}-\check{\xi}_{j}\right) \mathbf{1}_{\check{\xi}_{j}<T}
$$

so that for $\mathfrak{B}_{j}=\mathfrak{B}_{j}^{-} \cup \mathfrak{B}_{j}^{+}$,

$$
\sum_{\bar{t} \in \mathfrak{B}_{j}}\left|y^{\dagger}(\bar{t}-)-y^{\dagger}(\bar{t})\right| \leq 2^{-j+2} D\left(0,2^{-j}, \check{\xi}_{j-1}-\check{\xi}_{j}\right) \mathbf{1}_{\check{\xi}_{j}<T} .
$$

Including the possible jump-discontinuity of $y^{\dagger}$ at $\bar{t}=T$, and the contribution to the total variation of $y^{\dagger}$ from $[0, T] \backslash \cup_{j \in \mathbb{Z}} \mathfrak{B}_{j}$, we obtain

$$
\begin{aligned}
\left|y^{\dagger}\right|_{B V([0, T])} & \leq \sum_{j \in \mathbb{Z}}\left\{2^{-j+2} D\left(0,2^{-j}, \check{\xi}_{j-1}-\check{\xi}_{j}\right) \mathbf{1}_{\check{\xi}_{j}<T}\right\} \\
& +y^{\dagger}(T-)+\left|y^{\dagger}(T-)-y^{\dagger}(T)\right| \\
& \leq \sum_{j \in \mathbb{Z}}\left\{2^{-j+2} D\left(0,2^{-j}, \check{\xi}_{j-1}-\check{\xi}_{j}\right) \mathbf{1}_{\check{\xi}_{-j}<T}\right\}+2 M^{+}[z](T) .
\end{aligned}
$$

Observe that for all $j \in \mathbb{Z}$,

$$
Z_{j}=D\left(0,2^{-j}, \check{\xi}_{j-1}-\check{\xi}_{j}\right) \sim \operatorname{Geo}\left(\frac{1}{2}\right),
$$

$\mathbb{E}\left[Z_{j}\right]=1$ and $\mathbb{E}\left[Z_{j}^{2}\right]=3$, cf. (B.2. By [53, eq. (8.3)],

$$
\mathbb{P}\left(M^{+}[z](T) \in \mathrm{d} x\right)=\sqrt{\frac{2}{\pi T}} e^{-x^{2} /(2 T)} \mathrm{d} x, \quad x>0 .
$$


It therefore holds for all $j \geq 1$ that

$$
\begin{aligned}
\mathbb{P}\left(\check{\xi}_{-j}<T\right) & \leq \mathbb{P}\left(M^{+}[z](T) \geq 2^{j}\right)=\sqrt{\frac{2}{\pi T}} \int_{2^{j}}^{\infty} e^{-x^{2} /(2 T)} \mathrm{d} x \\
& <\sqrt{\frac{2}{\pi T}} \int_{2^{j}}^{\infty} \frac{8 T^{2}}{x^{4}} \mathrm{~d} x=\sqrt{\frac{128 T^{3}}{9 \pi}} 2^{-3 j}
\end{aligned}
$$

and

$$
\mathbb{E}\left[M^{+}[z](T)\right]=\sqrt{\frac{2}{\pi T}} \int_{0}^{\infty} x e^{-x^{2} /(2 T)} \mathrm{d} x=\sqrt{\frac{2 T}{\pi}} .
$$

By Hölder's inequality,

$$
\begin{aligned}
& \sum_{j \in \mathbb{Z}}^{\infty} \mathbb{E}\left[2^{-j+2} D\left(0,2^{-j}, \check{\xi}_{j-1}-\check{\xi}_{j}\right) \mathbf{1}_{\check{\xi}_{j}<T}\right] \\
& \leq \sum_{j \geq 0} \mathbb{E}\left[2^{-j+2} D\left(0,2^{-j}, \check{\xi}_{j-1}-\check{\xi}_{j}\right)\right]+\sum_{j<0} \mathbb{E}\left[2^{-j+2} D\left(0,2^{-j}, \check{\xi}_{j-1}-\check{\xi}_{j}\right) \mathbf{1}_{\check{\xi}_{j}<T}\right] \\
& =\sum_{j \geq 0} 2^{-j+2}+\sum_{j<0} 2^{-j+2} \sqrt{\mathbb{E}\left[\left|D\left(0,2^{-j}, \check{\xi}_{j-1}-\check{\xi}_{j}\right)\right|^{2}\right]} \sqrt{\mathbb{P}\left(\check{\xi}_{j}<T\right)} \\
& \leq 8+\left(\frac{128 T^{3}}{\pi}\right)^{1 / 4} \sum_{j \geq 1} 2^{2-j / 2}<8+25 T^{3 / 4} .
\end{aligned}
$$

By B.6 and the preceding inequality,

$$
\begin{aligned}
\mathbb{E}\left[\left|y^{\dagger}\right|_{B V([0, T])}\right] & \leq \mathbb{E}\left[\sum_{j \in \mathbb{Z}}^{\infty}\left\{2^{-j+2} D\left(0,2^{-j}, \check{\xi}_{j-1}-\check{\xi}_{j}\right) \mathbf{1}_{\check{\xi}_{-j}<T}\right\}+2 M^{+}[z](T)\right] \\
& =\sum_{j \in \mathbb{Z}}^{\infty} \mathbb{E}\left[2^{-j+2} D\left(0,2^{-j}, \check{\xi}_{j-1}-\check{\xi}_{j}\right) \mathbf{1}_{\check{\xi}_{-j}<T}\right]+\sqrt{\frac{8 T}{\pi}} \\
& \leq 8+25 T^{3 / 4}+\sqrt{\frac{8 T}{\pi}} .
\end{aligned}
$$

This verifies that

$$
\mathbb{E}\left[|\operatorname{orm}[z]|_{B V([0, T])}\right] \leq 2 \mathbb{E}\left[\left|y^{\dagger}\right|_{B V([0, T])}\right]<\infty,
$$

and thus, $|\operatorname{orm}[z]|_{B V([0, T])}<\infty, \mathbb{P}$-a.s.

A similar argument may be employed to verify that $\mathbb{E}\left[\sup _{m \geq 2}\left|\operatorname{orm}\left[z^{m}\right]\right|_{B V([0, T])}\right]<$ $\infty$. A short sketch of such an argument follows.

By symmetry, it suffices to verify that $y^{\dagger, m}=\mathbf{1}_{\omega \in \widetilde{\Omega}} \max \left(\operatorname{orm}\left[z^{m}\right], 0\right)$ has bounded total variation, uniformly in $m \geq 2, \mathbb{P}$-a.s. The only differing technicality from the preceding argument is that for fixed $m \geq 2$, any positive/negative jumpdiscontinuity of $y^{\dagger, m}$ at time $\bar{t}$ may be associated uniquely to a $z$-downcrossing of $\left[0,2^{k}\right]$ for some $k \geq j=\left\lfloor\log _{2}\left(M^{+}\left[z^{m}\right](\bar{t})\right)\right\rfloor$, in the time interval $\left[\check{\xi}_{k}, \check{\xi}_{k-1}\right]$ (i.e., through an injective mapping from the set of positive/negative jump-discontinuities of $y^{\dagger, m}$ to the set (B.4) ). Moreover $\left|y^{\dagger, m}(\bar{t}-)-y^{\dagger, m}(\bar{t})\right|<2^{j+1} \leq 2^{k+1}$. (The reason for $k \geq j$ in the association is that $z$-downcrossings may be more frequent than $z^{m}$ downcrossings, and they may also happen at other times.) It consequently holds that

$$
\sup _{m \geq 2}\left|y^{\dagger, m}\right|_{B V([0, T])} \leq \sum_{j \in \mathbb{Z}}\left\{2^{-j+2} D\left(0,2^{-j}, \check{\xi}_{j-1}-\check{\xi}_{j}\right) \mathbf{1}_{\check{\xi}_{j}<T}\right\}+2 M^{+}[z](T),
$$

and the result follows. 


\section{REFERENCES}

[1] R. Abgrall and S. Mishra. Uncertainty quantification for hyperbolic systems of conservation laws. In Handbook of Numerical Analysis, volume 18, pages 507-544. Elsevier, 2017.

[2] S. Attanasio and F. Flandoli. Renormalized solutions for stochastic transport equations and the regularization by bilinear multiplication noise. Comm. Partial Differential Equations, 36(8):1455-1474, 2011.

[3] I. Babuška, F. Nobile, and R. Tempone. A stochastic collocation method for elliptic partial differential equations with random input data. SIAM Journal on Numerical Analysis, 45(3):1005-1034, 2007.

[4] I. Bailleul and M. Gubinelli. Unbounded rough drivers. ArXiv e-prints, Jan. 2015.

[5] C. Bauzet. Time-splitting approximation of the cauchy problem for a stochastic conservation law. Mathematics and Computers in Simulation, 118:73-86, 2015.

[6] C. Bauzet, J. Charrier, and T. Gallouët. Convergence of flux-splitting finite volume schemes for hyperbolic scalar conservation laws with a multiplicative stochastic perturbation. Math. Comp., 85(302):2777-2813, 2016.

[7] C. Bauzet, J. Charrier, and T. Gallouët. Convergence of monotone finite volume schemes for hyperbolic scalar conservation laws with multiplicative noise. Stoch. Partial Differ. Equ. Anal. Comput., 4(1):150-223, 2016.

[8] C. Bauzet, G. Vallet, and P. Wittbold. The Cauchy problem for conservation laws with a multiplicative stochastic perturbation. J. Hyperbolic Differ. Equ., 9(4):661-709, 2012.

[9] C. Bayer, P. K. Friz, S. Riedel, and J. Schoenmakers. From rough path estimates to multilevel monte carlo. SIAM Journal on Numerical Analysis, 54(3):1449-1483, 2016.

[10] L. Benigni, C. Cosco, A. Shapira, and K. J. Wiese, Hausdorff Dimension of the Record Set of a Fractional Brownian, arXiv preprint arXiv:1706.09726, 2017.

[11] I. H. Biswas, K. H. Karlsen, and A. K. Majee. Conservation laws driven by Lévy white noise. J. Hyperbolic Differ. Equ., 12(3):581-654, 2015.

[12] Y. Brenier and S. Osher. The discrete one-sided Lipschitz condition for convex scalar conservation laws. SIAM J. Numer. Anal., 25(1):8-23, 1988 .

[13] G.-Q. Chen and K. H. Karlsen. Quasilinear anisotropic degenerate parabolic equations with time-space dependent diffusion coefficients. Commun. Pure Appl. Anal., 4(2):241-266, 2005.

[14] G.-Q. Chen, Q. Ding, and K. H. Karlsen. On nonlinear stochastic balance laws. Arch. Ration. Mech. Anal., 204(3):707-743, 2012.

[15] K. A. Cliffe, M. B. Giles, R. Scheichl, and A. L. Teckentrup. Multilevel monte carlo methods and applications to elliptic pdes with random coefficients. Computing and Visualization in Science, 14(1):3, 2011.

[16] C. M. Dafermos. Characteristics in hyperbolic conservation laws. A study of the structure and the asymptotic behaviour of solutions. pages 1-58. Res. Notes in Math., No. 17, 1977.

[17] C. M. Dafermos. Regularity and large time behaviour of solutions of a conservation law without convexity. Proc. Roy. Soc. Edinburgh Sect. A, 99(3-4):201-239, 1985.

[18] C. M. Dafermos. Hyperbolic conservation laws in continuum physics, volume 325 of Grundlehren der Mathematischen Wissenschaften [Fundamental Principles of Mathematical Sciences]. Springer-Verlag, Berlin, third edition, 2010.

[19] A. Debussche and J. Vovelle. Scalar conservation laws with stochastic forcing. J. Funct. Anal., 259(4):1014-1042, 2010.

[20] A. Debussche and J. Vovelle. Invariant measure of scalar first-order conservation laws with stochastic forcing. Probab. Theory Related Fields, 163(3-4):575-611, 2015.

[21] A. Debussche, M. Hofmanová, and J. Vovelle. Degenerate parabolic stochastic partial differential equations: Quasilinear case. Ann. Probab., 44(3):1916-1955, 2016.

[22] A. Deya, M. Gubinelli, M. Hofmanová, and S. Tindel. A priori estimates for rough PDEs with application to rough conservation laws. ArXiv e-prints, Apr. 2016.

[23] S. Dotti and J. Vovelle. Convergence of approximations to stochastic scalar conservation laws. ArXiv e-prints, Nov. 2016.

[24] S. Dotti and J. Vovelle. Convergence of the Finite Volume Method for scalar conservation laws with multiplicative noise: an approach by kinetic formulation. ArXiv e-prints, Nov. 2016.

[25] R. M. Dudley. Sample functions of the Gaussian process. Ann. Probab., 1(1):66-103, 1973.

[26] Benjamin Gess and Panagiotis E Souganidis. Long-time behavior, invariant measures, and regularizing effects for stochastic scalar conservation laws. Communications on Pure and Applied Mathematics, 70(8):1562-1597, 2017.

[27] A. Gut. Probability: a graduate course. Springer Texts in Statistics. Springer, New York, second edition, 2013.

[28] W. E, K. Khanin, A. Mazel, and Y. Sinai. Invariant measures for Burgers equation with stochastic forcing. Ann. of Math. (2), 151(3):877-960, 2000. 
[29] L. C. Evans. An introduction to stochastic differential equations. American Mathematical Society, Providence, RI, 2013.

[30] J. Feng and D. Nualart. Stochastic scalar conservation laws. J. Funct. Anal., 255(2):313-373, 2008.

[31] F. Flandoli, M. Gubinelli, and E. Priola. Well-posedness of the transport equation by stochastic perturbation. Invent. Math., 180(1):1-53, 2010.

[32] J. G. Gaines and T. J. Lyons. Variable step size control in the numerical solution of stochastic differential equations. SIAM Journal on Applied Mathematics, 57(5):1455-1484, 1997.

[33] P. Gassiat and B. Gess. Regularization by noise for stochastic Hamilton-Jacobi equations. Probability Theory and Related Fields, pages 1-36, 2016.

[34] P. Gassiat, B. Gess, P.-L. Lions, and P. E. Souganidis. Speed of propagation for HamiltonJacobi equations with multiplicative rough time dependence and convex hamiltonians. ArXiv e-prints, 2018.

[35] B. Gess, B. Perthame, and P. E. Souganidis. Semi-discretization for stochastic scalar conservation laws with multiple rough fluxes. SIAM J. Numer. Anal., 54(4):2187-2209, 2016.

[36] B. Gess and P. E. Souganidis. Long-time behavior, invariant measures and regularizing effects for stochastic scalar conservation laws. Communications on Pure and Applied Mathematics, 70(8):1562-1597, 2017.

[37] B. Gess and P. E. Souganidis. Scalar conservation laws with multiple rough fluxes. Commun. Math. Sci., 13(6):1569-1597, 2015.

[38] M. B. Giles. Multilevel monte carlo methods. Acta Numerica, 24:259-328, 2015.

[39] M. B. Giles, C. Lester, and J. Whittle. Non-nested adaptive timesteps in multilevel Monte Carlo computations. In Monte Carlo and Quasi-Monte Carlo Methods, pages 303-314. Springer, 2016.

[40] A.-L. Haji-Ali, F. Nobile, and R. Tempone. Multi-index Monte Carlo: when sparsity meets sampling. Numerische Mathematik, 132(4):767-806, 2016.

[41] A.-L. Haji-Ali, F. Nobile, E. von Schwerin, and R. Tempone. Optimization of mesh hierarchies in multilevel Monte Carlo samplers. Stochastics and Partial Differential Equations Analysis and Computations, 4(1):76-112, 2016.

[42] E. J. Hall, H. Hoel, M. Sandberg, A. Szepessy, and R. Tempone. Computable error estimates for finite element approximations of elliptic partial differential equations with rough stochastic data. SIAM Journal on Scientific Computing, 38(6):A3773-A3807, 2016.

[43] M. Hardy. Combinatorics of partial derivatives. Electron. J. Combin., 13(1):Research Paper 1,13 pp. (electronic), 2006.

[44] R. Hartmann and P. Houston. Adaptive discontinuous galerkin finite element methods for nonlinear hyperbolic conservation laws. SIAM Journal on Scientific Computing, 24(3):979$1004,2003$.

[45] H. Hoel, J. Häppölä, and R. Tempone. Construction of a mean square error adaptive eulermaruyama method with applications in multilevel Monte Carlo. In Monte Carlo and QuasiMonte Carlo Methods, pages 29-86. Springer, 2016.

[46] H. Hoel, K. H. Karlsen, N. H. Risebro, and E. B. Storrøsten. Path-dependent convex conservation laws. J. Differential Equations, 265(6):2708-2744, 2018.

[47] H. Hoel, E. Von Schwerin, A. Szepessy, and R. Tempone. Implementation and analysis of an adaptive multilevel Monte Carlo algorithm. Monte Carlo Methods and Applications, 20(1):141, 2014.

[48] M. Hofmanová. Degenerate parabolic stochastic partial differential equations. Stochastic Process. Appl., 123(12):4294-4336, 2013.

[49] M. Hofmanová. Scalar conservation laws with rough flux and stochastic forcing. Stoch. Partial Differ. Equ. Anal. Comput., 4(3):635-690, 2016.

[50] H. Holden and N. H. Risebro. Conservation laws with a random source. Appl. Math. Optim., 36(2):229-241, 1997.

[51] H. Holden and N. H. Risebro. Front tracking for hyperbolic conservation laws, volume 152 of Applied Mathematical Sciences. Springer, Heidelberg, second edition, 2015.

[52] C. Johnson and A. Szepessy. Adaptive finite element methods for conservation laws based on a posteriori error estimates. Communications on Pure and Applied Mathematics, 48(3):199234,1995

[53] I. Karatzas and S. E. Shreve. Brownian Motion and Stochastic Calculus, volume 113. Springer Science \& Business Media, 1991.

[54] K. H. Karlsen and N. H. Risebro. On the uniqueness and stability of entropy solutions of nonlinear degenerate parabolic equations with rough coefficients. Discrete Contin. Dyn. Syst., 9(5):1081-1104, 2003.

[55] K. H. Karlsen and E. B. Storrøsten. On stochastic conservation laws and Malliavin calculus. J. Funct. Anal., 272:421-497, 2017. 
[56] K. H. Karlsen and E. B. Storrøsten. Analysis of a splitting method for stochastic balance laws. IMA J. Numer. Anal., 38(1):1-56, 2018.

[57] C. Kelly and G. J. Lord. Adaptive time-stepping strategies for nonlinear stochastic systems. IMA Journal of Numerical Analysis, 2016.

[58] J. U. Kim. On a stochastic scalar conservation law. Indiana Univ. Math. J. 52 (1) (2003) $227-256$.

[59] D. P. Kroese and Z. I. Botev. Spatial process simulation. In Stochastic Geometry, Spatial Statistics and Random Fields, pages 369-404. Springer, 2015.

[60] I. Kröker and C. Rohde. Finite volume schemes for hyperbolic balance laws with multiplicative noise. Appl. Numer. Math., 62(4):441-456, 2012.

[61] D. Kröner. Numerical schemes for conservation laws. Wiley-Teubner Series Advances in Numerical Mathematics. John Wiley \& Sons Ltd., Chichester, 1997.

[62] P.-L. Lions, B. Perthame, and P. Souganidis. Scalar conservation laws with rough (stochastic) fluxes. Stoch. Partial Differ. Equ. Anal. Comput., 1(4):664-686, 2013.

[63] P.-L. Lions, B. Perthame, and P. E. Souganidis. Stochastic averaging lemmas for kinetic equations. ArXiv e-prints, Apr. 2012.

[64] P.-L. Lions, B. Perthame, and P. E. Souganidis. Scalar conservation laws with rough (stochastic) fluxes: the spatially dependent case. Stoch. Partial Differ. Equ. Anal. Comput., $2(4): 517-538,2014$.

[65] B. J. Lucier. A moving mesh numerical method for hyperbolic conservation laws. Math. Comp., 46(173):59-69, 1986.

[66] J. Málek, J. Nečas, M. Rokyta, and M. Růžička. Weak and measure-valued solutions to evolutionary PDEs, volume 13 of Applied Mathematics and Mathematical Computation. Chapman \& Hall, London, 1996.

[67] S. Mishra and C. Schwab. Sparse tensor multi-level Monte Carlo finite volume methods for hyperbolic conservation laws with random initial data. Mathematics of Computation, 81(280):1979-2018, 2012.

[68] S. Mishra, C. Schwab, and J. Šukys. Multi-level Monte Carlo finite volume methods for nonlinear systems of conservation laws in multi-dimensions. Journal of Computational Physics, 231(8):3365-3388, 2012.

[69] S.-E. A. Mohammed, T. K. Nilssen, and F. N. Proske. Sobolev differentiable stochastic flows for SDEs with singular coefficients: Applications to the transport equation. Ann. Probab., 43(3):1535-1576, 2015.

[70] P. Mörters and Y. Peres. Brownian motion. Cambridge Series in Statistical and Probabilistic Mathematics. Cambridge University Press, Cambridge, 2010.

[71] W. Neves and C. Olivera. Wellposedness for stochastic continuity equations with Ladyzhenskaya-Prodi-Serrin condition. NoDEA Nonlinear Differential Equations Appl., 22(5):1247-1258, 2015.

[72] B. Perthame. Kinetic formulation of conservation laws, volume 21 of Oxford Lecture Series in Mathematics and its Applications. Oxford University Press, Oxford, 2002.

[73] N. H. Risebro, C. Schwab, and F. Weber. Multilevel Monte Carlo front-tracking for random scalar conservation laws. BIT Numerical Mathematics, 56(1):263-292, 2016.

[74] B. Seeger. Approximation schemes for viscosity solutions of fully nonlinear stochastic partial differential equations. ArXiv e-prints, 2018.

[75] A. Szepessy, R. Tempone, and G. E. Zouraris. Adaptive weak approximation of stochastic differential equations. Communications on Pure and Applied Mathematics, 54(10):1169-1214, 2001.

[76] G. Vallet. Dirichlet problem for a nonlinear conservation law. Rev. Mat. Complut., 13(1):231250, 2000.

[77] G. Vallet and P. Wittbold. On a stochastic first-order hyperbolic equation in a bounded domain. Infin. Dimens. Anal. Quantum Probab. Relat. Top., 12(4):613-651, 2009.

[78] D. Xiu and J. S. Hesthaven. High-order collocation methods for differential equations with random inputs. SIAM Journal on Scientific Computing, 27(3):1118-1139, 2005.

[79] L. Yaroslavtseva. On non-polynomial lower error bounds for adaptive strong approximation of sdes. Journal of Complexity, 42:1-18, 2017. 
(Håkon Hoel)

Mathematics Institute of Computational Science and Engineering

ÉCOLE POLYTECHNique FÉdÉRALE DE LAUSANNE

EPFL / SB / MATH-CSQI, MA C1 644 (BÂtiment MA), Station 8

CH-1015, Lausanne

SWITZERLAND

Email address: hakon.hoel@epfl.ch, hhakon@chalmers.se

(Kenneth Hvistendahl Karlsen)

DEPARTMENT OF MATHEMATICS

UNIVERSITY OF OSLO

P.O. Box 1053, BLINDERN

N-0316 OSLO, NorwaY

Email address: kennethk@math.uio.no

(Nils Henrik Risebro)

Department of MATHEMATiCs

UNIVERSITY OF OSLO

P.O. Box 1053, BLINDERN

N-0316 Oslo, Norway

Email address: nilsr@math.uio.no

(Erlend Briseid Storrøsten)

DEPARTMENT OF MATHEMATICS

UNIVERSITY OF OSLO

P.O. Box 1053, Blindern

N-0316 Oslo, Norway

Email address: erlenbs@math.uio.no 